\title{
Gene editing and its applications in biomedicine
}

\author{
Guanglei $\mathrm{Li}^{1 \dagger}$, Xiangyang $\mathrm{Li}^{1 \dagger}$, Songkuan Zhuang ${ }^{2 \dagger}$, Liren Wang ${ }^{3 \dagger}$, Yifan $\mathrm{Zhu}^{3 \dagger}$, \\ Yangcan Chen ${ }^{4,5,6 \dagger}$, Wen Sun ${ }^{4,6 \dagger}$, Zeguang $\mathrm{Wu}^{7 \dagger}$, Zhuo Zhou ${ }^{7 \dagger}$, Jia Chen ${ }^{8^{*}}$, Xingxu Huang ${ }^{{ }^{*}}$, \\ Jin Wang ${ }^{2^{*}}$, Dali $\mathrm{Li}^{3^{*}}$, Wei $\mathrm{Li}^{4,5,6,9,10^{*}}$, Haoyi Wang ${ }^{4,5,6^{*}}$ \& Wensheng Wei ${ }^{7^{*}}$ \\ ${ }^{1}$ School of Life Science and Technology, ShanghaiTech University, Shanghai 201210, China; \\ ${ }^{2}$ Department of Clinical Laboratory, Shenzhen Institute of Translational Medicine, The First Affiliated Hospital of Shenzhen University, \\ Shenzhen Second People's Hospital, Shenzhen 518035, China; \\ ${ }^{3}$ Shanghai Frontiers Science Research Base of Genome Editing and Cell Therapy, Shanghai Key Laboratory of Regulatory Biology, School of \\ Life Sciences, East China Normal University, Shanghai 200241, China; \\ ${ }^{4}$ State Key Laboratory of Stem Cell and Reproductive Biology, Institute of Zoology, Chinese Academy of Sciences, Beijing 100101, China; \\ ${ }^{5}$ University of Chinese Academy of Sciences, Beijing 100049, China; \\ ${ }^{6}$ Institute for Stem Cell and Regenerative Medicine, Chinese Academy of Sciences, Beijing 100101, China; \\ ${ }^{7}$ Biomedical Pioneering Innovation Center, Beijing Advanced Innovation Center for Genomics, Peking-Tsinghua Center for Life Sciences, \\ Peking University Genome Editing Research Center, State Key Laboratory of Protein and Plant Gene Research, School of Life Sciences, \\ Peking University, Beijing 100871, China, \\ ${ }^{8}$ Gene Editing Center, School of Life Science and Technology, ShanghaiTech University, Shanghai 201210, China; \\ ${ }^{9}$ Beijing Institute for Stem Cell and Regenerative Medicine, Beijing 100101, China; \\ ${ }^{10}$ HIT Center for Life Sciences, Harbin Institute of Technology, Harbin 150001, China
}

Received October 27, 2021; accepted December 6, 2021; published online February 18, 2022

\begin{abstract}
The steady progress in genome editing, especially genome editing based on the use of clustered regularly interspaced short palindromic repeats (CRISPR) and programmable nucleases to make precise modifications to genetic material, has provided enormous opportunities to advance biomedical research and promote human health. The application of these technologies in basic biomedical research has yielded significant advances in identifying and studying key molecular targets relevant to human diseases and their treatment. The clinical translation of genome editing techniques offers unprecedented biomedical engineering capabilities in the diagnosis, prevention, and treatment of disease or disability. Here, we provide a general summary of emerging biomedical applications of genome editing, including open challenges. We also summarize the tools of genome editing and the insights derived from their applications, hoping to accelerate new discoveries and therapies in biomedicine.
\end{abstract}

gene editing, CRISPR, high-throughput functional genomics, diagnostics, animal model, therapeutics

Citation: Li, G., Li, X., Zhuang, S., Wang, L., Zhu, Y., Chen, Y., Sun, W., Wu, Z., Zhou, Z., Chen, J., et al. (2022). Gene editing and its applications in biomedicine. Sci China Life Sci 65, 660-700. https://doi.org/10.1007/s11427-021-2057-0

$\uparrow$ Contributed equally to this work

*Corresponding authors (Jia Chen, email: chenjia@shanghaitech.edu.cn; Xingxu Huang, email: huangxx@shanghaitech.edu.cn; Jin Wang, email: wangj01@hotmail.com; Dali Li, email: dlli@bio.ecnu.edu.cn; Wei Li, email: liwei@ioz.ac.cn; Haoyi Wang, email: wanghaoyi@ioz.ac.cn; Wensheng Wei, email: wswei@pku.edu.cn) 


\section{Introduction}

The purpose of gene-editing technology is to precisely change DNA sequences at target sites. By fusing zinc finger (ZF) proteins or transcription activator-like effector proteins with the cleavage domain of FokI endonuclease, zinc finger nucleases (ZFNs) (Beerli and Barbas, 2002) or transcription activator-like effector nucleases (TALENs) (Boch and Bonas, 2010) were developed, respectively, which started the era of programmable gene editing. More recently, under the direction of a guide RNA (gRNA), clustered regularly interspaced short palindromic repeats (CRISPR)/CRISPR-associated protein (Cas) nuclease can cleave the DNA doublestrand at target sites with great convenience, efficiency, and precision (Chang et al., 2013; Cong et al., 2013; Jinek et al., 2012; Jinek et al., 2013; Mali et al., 2013).

Double-stranded breaks (DSBs) in genomic DNA are repaired by two endogenous pathways: nonhomologous end joining (NHEJ) and homology-directed repair (HDR) (Cec- caldi et al., 2016). NHEJ introduces random insertions or deletions (indels) of nucleotides, which often lead to open reading frame shift mutations (Deriano and Roth, 2013) and ultimately disrupt the expression of the target gene (so-called "knockout"). Alternatively, precise sequence replacement or insertion (so-called "knockin") can be achieved via HDR when donor DNA is present (Jasin and Rothstein, 2013). However, HDR efficiency is normally low in many types of cells, limiting the breadth of its applications in biomedical research and gene therapy. Recently, by fusing nuclease activity-impaired Cas proteins with different effector modules, including nucleobase deaminase and reverse transcriptase, base editors (BEs) (Gaudelli et al., 2017; Komor et al., 2016) and prime editors (PEs) (Anzalone et al., 2019) have been developed to achieve precise editing with high efficiency and product purity.

This section reviews the development and improvement of various gene-editing technologies, including ZFN, TALEN, CRISPR/Cas, BE, PE, and RNA editing (Table 1).

Table 1 Representative editors

\begin{tabular}{|c|c|c|c|c|c|c|}
\hline Genome editor & Targeting ability & Knock out & Knock in & Base substitution & $\begin{array}{l}\text { Off-target } \\
\text { effects }\end{array}$ & Reference \\
\hline ZFN & + & +++ & + & + & +++ & $\begin{array}{c}\text { (Bibikova et al., 2002; Kim et } \\
\text { al., 1996) }\end{array}$ \\
\hline TALEN & ++ & +++ & + & + & +++ & $\begin{array}{l}\text { (Cermak et al., 2011; Christian } \\
\text { et al., 2010; Miller et al., 2011) }\end{array}$ \\
\hline Cas9 & +++ (NGG PAM) & +++ & + & + & +++ & $\begin{array}{l}\text { (Cong et al., 2013; Jinek et al., } \\
\text { 2013; Mali et al., 2013) }\end{array}$ \\
\hline Cas12a & +++ (NGG PAM) & +++ & + & + & ++ & (Zetsche et al., 2015) \\
\hline nCas9 & $\begin{array}{l}++ \text { (sgRNA pair, } \\
\text { NGG PAM) }\end{array}$ & ++ & + & + & ++ & $\begin{array}{l}\text { (Ran et al., 2013; } \\
\text { Shen et al., 2014) }\end{array}$ \\
\hline dCas9-FokI & $\begin{array}{l}++ \text { (sgRNA pair, } \\
\text { NGG PAM) }\end{array}$ & ++ & + & + & + & $\begin{array}{l}\text { (Guilinger et al., 2014; } \\
\text { Tsai et al., 2014) }\end{array}$ \\
\hline BE3 & ++ (NGG PAM) & $\begin{array}{l}+++ \text { (Creating stop } \\
\text { codon) }\end{array}$ & - & $+++($ C-to-T/G-to-A $)$ & +++ & (Komor et al., 2016) \\
\hline hA3A-BE3 & ++ (NGG PAM) & $\begin{array}{l}+++ \text { (Creating stop } \\
\text { codon })\end{array}$ & - & $+++($ C-to-T/G-to-A $)$ & +++ & (Wang et al., 2018e) \\
\hline dCas12a-BE & ++ (TTTV PAM) & $\begin{array}{l}+++ \text { (Creating stop } \\
\text { codon) }\end{array}$ & - & $+++($ C-to-T/G-to-A) & ++ & (Li et al., 2018d) \\
\hline BEACON & ++ (TTTV PAM) & $\begin{array}{c}\text { +++eating stop } \\
\text { codon })\end{array}$ & - & $+++($ C-to-T/G-to-A $)$ & + & (Wang et al., 2020f) \\
\hline $\mathrm{tBE}$ & $\begin{array}{c}++ \text { (NGG or NG } \\
\text { PAM) }\end{array}$ & $\begin{array}{c}+++ \text { (Creating stop } \\
\text { codon) }\end{array}$ & - & $+++($ C-to-T/G-to-A $)$ & - & (Wang et al., 2021) \\
\hline ABE7.10 & $++($ NGG PAM $)$ & $\begin{array}{c}\text { +++ (Mutating splicing } \\
\text { site) }\end{array}$ & - & $+++($ A-to-G/T-to-C) & +++ & (Gaudelli et al., 2017) \\
\hline LbABE8e & ++ (TTTV PAM) & $\begin{array}{c}\text { +++ (Mutating splicing } \\
\text { site) }\end{array}$ & - & $+++($ A-to-G/T-to-C) & ++ & (Richter et al., 2020) \\
\hline PE3 & ++ (NGG PAM) & $\begin{array}{l}+++ \text { (Creating stop } \\
\text { codon })\end{array}$ & $++($ Small insertion $)$ & ++ & $+/-$ & (Anzalone et al., 2019) \\
\hline Cas 13 & $\begin{array}{l}+++\left(\text { RNA, no PFS }{ }^{\mathrm{a})}\right. \\
\text { constraint })\end{array}$ & ++ (Knock down) & - & - & ++ & (Abudayyeh et al., 2017) \\
\hline REPAIR & $\begin{array}{c}++ \text { (RNA, no PFS } \\
\text { constraint) }\end{array}$ & $\begin{array}{l}+(\text { Knock down, } \\
\text { mutating start codon })\end{array}$ & - & $++($ A-to-G/T-to-C) & ++ & (Cox et al., 2017) \\
\hline RESCUE & $\begin{array}{c}++ \text { (RNA, no PFS } \\
\text { constraint) }\end{array}$ & $\begin{array}{l}++(\text { Knock down, } \\
\text { creating stop codon })\end{array}$ & - & $++($ C-to-T/G-to-A $)$ & ++ & (Abudayyeh et al., 2019) \\
\hline
\end{tabular}

a) PFS: protospacer flanking site. 


\section{ZFN}

Site-specific nucleases have long been applied in DNA recombination in vitro, and therefore, these nucleases were first used for gene editing. Meganucleases, a type of endonuclease that recognizes long DNA sequences (e.g., $\sim 12-40 \mathrm{bp}$ ), have been applied and engineered to generate DSBs at genomic loci. However, meganucleases have not been used widely in genome editing due to their limited recognition sites and the difficulty of designing their targeting specificity. However, DNA endonucleases can be used as effector modules to generate DNA breaks. The FokI endonuclease in particular has separate domains responsible for DNA binding and cleavage. A single DNA cleavage domain of FokI is inactive, but the dimerization of two cleavage domains gains DNA cleavage activity and cuts DNA double strands with no sequence specificity. Thus, a pair of fusion proteins, each containing a FokI DNA cleavage domain and a locator module, can be used for targeted gene editing.

Zinc finger motifs, originally discovered in transcription factors in Xenopus laevis (Klug, 2010; Miller et al., 1985), were used as the first locators for DNA targeting in gene editing. One ZF motif binds to three base pairs (bp) and an array of ZF motifs that recognize $\sim 9-18$ bp of specific DNA sequences (Bibikova et al., 2002). This modular configuration makes ZF a potential platform for programmable genome targeting. By fusing an array of ZF motifs with the FokI cleavage domain, ZFNs were developed for programmable gene editing (Kim et al., 1996). Generally, a pair of ZFNs that target the upstream and downstream regions of an intended genomic locus can be used to induce the dimerization of FokI cleavage domains, which then cleave DNA double strands. The repair of DSBs by NHEJ or HDR can eventually lead to random indels or precise sequence replacement for gene knockout or knockin, respectively.

Although some customized ZF arrays can efficiently bind to targeted genomic loci, the construction of $\mathrm{ZF}$ arrays for most genomic sites remains challenging, as the crosstalk between adjacent ZF motifs can interfere with the binding of a ZF array to the intended DNA region. Thus, the generation of a pair of functional ZFNs requires the screening of numerous ZF arrays. As a potential gene therapy tool, ZFNs have relatively small sizes and can be packaged into adenoassociated virus (AAV) (Yin et al., 2017a), a convenient and prevalent vector for in vivo gene editing.

Off-target (OT) editing, changes in the DNA sequence at unintended genomic sites, is one of the major concerns about genome editing technology, especially for its applications in gene therapy. The OT effects of ZFNs have been systematically examined in vitro, and obvious cleavage can be induced by ZFN at hundreds of OT sites (Pattanayak et al., 2011). Some of the in vitro identified OT sites can be edited by ZFNs in human cells, confirming the OT effects of ZFNs in vivo.

\section{TALEN}

Discovered in the bacterial plant pathogen Xanthomonas, transcription activator-like effector (TALE) proteins contain DNA binding domains that are repeats of amino acid residues (Boch and Bonas, 2010). The DNA binding domain of TALE has tandem 33-34 amino acid repeats with divergent dual residues at positions 12 and 13 . These two positions (the so-called repeat variable diresidue, RVD) are highly variable and determine the DNA binding specificity of a TALE protein (Boch et al., 2009; Moscou and Bogdanove, 2009). As a TALE motif containing a specific RVD can recognize a specific nucleotide, a combination of repeat TALE motifs containing the appropriate RVDs can bind to a specific DNA sequence. In contrast to ZF arrays, TALE arrays can recognize and bind to target sites without interfering with each TALE domain in the array.

Similar to ZFN, by taking advantage of a pair of fusion proteins of the FokI cleavage domain and TALE array, TALENs were developed to induce DSBs at targeted genomic sites (Cermak et al., 2011; Miller et al., 2011). Although a pair of functional TALENs can be generated without tedious screening of TALE arrays, the construction of TALEN-expressing vectors is still complicated due to homologous recombination between repetitive TALE sequences. In addition, the complex design and construction of TALENexpressing vectors are time-consuming and costly, which also hampers some of their potential applications, such as high-throughput assays. Although a study showed that the incidence of OT editing of TALEN in human stem cells was low (Veres et al., 2014), the systematic analysis of OT effects by TALEN awaits further investigation.

\section{CRISPR-Cas}

CRISPR/Cas was originally identified as a defense system in bacteria to provide acquired immunity against bacterial parasites, such as bacteriophages and plasmids (Barrangou et al., 2007; Bolotin et al., 2005; Mojica et al., 2005; Pourcel et al., 2005). Major types of CRISPR/Cas used for gene editing belong to the class 2 system (Makarova et al., 2020), which requires only one DNA endonuclease, e.g., Cas9 (Cong et al., 2013; Jinek et al., 2012; Mali et al., 2013) or Cas12a (also known as Cpf1) (Zetsche et al., 2015), to cleave double strands of bacteriophage or plasmid DNA under the guidance of CRISPR RNA (crRNA). In the CRISPR/Cas9 system, a crRNA and a trans-activating crRNA (tracrRNA) form a double-stranded RNA, which can be processed by RNase III, and then the mature crRNA/tracrRNA complex recruits Cas9 protein to form a ribonucleoprotein (RNP) complex 
(Gasiunas et al., 2012; Jinek et al., 2012). Single-guide RNA (sgRNA) was engineered by fusing crRNA to scaffold tracrRNA to recruit Cas9 to facilitate gene editing in various species (Jinek et al., 2012). In the CRISPR/Cas12a system, only crRNA is needed to recruit the Cas 12 a protein to form an RNP complex (Zetsche et al., 2015). Directed by an sgRNA or a crRNA, the Cas9 or Cas12a RNP complex binds to the target site, which is complementary to the spacer region of the corresponding sgRNA or crRNA and has a protospacer-adjacent motif (PAM) (Anders et al., 2014; Nishimasu et al., 2014; Sternberg et al., 2014; Zetsche et al., 2015). Generally, different Cas proteins recognize different PAM sequences. For instance, the commonly used Streptococcus pyogenes Cas9 (SpCas9) recognizes an NGG PAM sequence at the $3^{\prime}$ end of the protospacer region (Jinek et al., 2012; Ran et al., 2015), while Acidaminococcus Cas12a (AsCas12a) and Lachnospiraceae bacterium Cas12a (LbCas12a) recognize a TTTV PAM sequence at the $5^{\prime}$ end of the protospacer region (Zetsche et al., 2015). After binding at a target site, the $\mathrm{HNH}$ and RuvC-like endonuclease domains of Cas9 cleave target (complementary) and nontarget (noncomplementary) DNA strands, respectively (Jinek et al., 2012). The cleavage results in two blunt DNA ends, and the cleavage site is $3 \mathrm{bp}$ upstream of the PAM sequence (Jinek et al., 2012). In contrast, Cas12a proteins have only a RuvC-like endonuclease domain, and they generate sticky DNA ends distal to the PAM sequence (Zetsche et al., 2015). The discovery of new Cas proteins and the engineering of discovered Cas proteins continuously expand the DNA targeting range of CRISPR/Cas systems (Hu et al., 2018; Kleinstiver et al., 2015; Miller et al., 2020; Nishimasu et al., 2018).

Genome-wide analyses have shown that Cas9 endonuclease can bind and cleave DNA double strands at OT sites with sequence similarity to the on-target sites (Kim et al., 2015; Tsai et al., 2015; Tsai et al., 2017). Generally, mismatches between the sgRNA spacer region and OT site can be better tolerated at the PAM-distal region than at the PAM-proximal region. Although the use of sgRNA with fewer potential OT sites in genomic DNA can reduce the OT effects of Cas9, the development of an improved CRISPR/ Cas9 system with high editing specificity substantially reduced its OT effects. As Cas9 proteins contain two endonuclease domains, one endonuclease domain can be mutated, which results in a nickase version of Cas9 (nCas9). nCas 9 can be used for gene knockout when it is co-expressed with a pair of sgRNAs targeting the opposite DNA strands of an on-target site (Ran et al., 2013). In this situation, nCas9 generates two nicks at opposite DNA strands, which mimics a DSB. In contrast, nCas9 generates only a DNA singlestrand break (SSB) at a particular OT site as two sgRNAs have distinct OT sites, and thus the OT indels triggered by DSBs are largely avoided. However, SSBs can still induce some levels of indels at certain OT sites, as an SSB can be converted to a DSB through endogenous DNA repair processes involving endogenously expressed cytidine deaminases (Lei et al., 2018). The FokI cleavage domain was fused to catalytically dead Cas9 (dCas9) to decrease OT editing further. The fusion protein can induce DSBs at on-target sites where a pair of sgRNAs induce the dimerization and activation of FokI nuclease; however, no DNA break is generated at OT sites where the FokI cleavage domain remains inactive as a monomer (Fu et al., 2014; Guilinger et al., 2014).

Another strategy for improving editing specificity is to engineer Cas9 proteins. The residues of Cas9 that are involved in the interaction with the DNA backbone were mutated to reduce the binding of Cas9 at OT sites, while the binding and editing ability at on-target sites was largely retained (Chen et al., 2017a; Kleinstiver et al., 2016a; Lee et al., 2018; Slaymaker et al., 2016). In addition, sgRNAs for Cas 9 have been modified to reduce OT effects, for instance, changing the length of the spacer region (Fu et al., 2014; Kim et al., 2015) or adding an RNA secondary structure onto the $5^{\prime}$ end of an sgRNA (Kocak et al., 2019). The method of delivery also affects the specificity of CRISPR/Cas9-mediated gene editing. Generally, the delivery of RNP complexes or RNAs provides higher editing specificity than DNA delivery, as the continuous expression of sgRNA and Cas9 from plasmid DNA can increase editing at OT sites (Kim et al., 2014; Ramakrishna et al., 2014; Rees et al., 2017; Yin et al., 2017b).

As ZFN, TALEN, and CRISPR/Cas all generate DSBs to initiate genome editing, the DNA damage response (DDR) triggered by DSBs has been observed in an increasing number of studies (Haapaniemi et al., 2018; Ihry et al., 2018). As a toxic DNA lesion, DSBs can trigger the phosphorylation and activation of a key DDR, ataxia-telangiectasia mutated (ATM) (Shiloh and Ziv, 2013). Furthermore, the end resection in the DSB repair process can generate single-stranded DNA regions, triggering the phosphorylation and activation of additional key DDR effectors, ATM and RAD3-related (ATR) protein kinase (Cimprich and Cortez, 2008). Both activated ATM and ATR can subsequently phosphorylate p53, resulting in cell cycle arrest or even cell death (Khanna et al., 1998; Tibbetts et al., 1999). Thus, preventing DDR would be a future direction to further improve gene-editing technology.

\section{Base editor}

The apolipoprotein B mRNA editing enzyme, catalytic polypeptide-like (APOBEC), and the activation-induced deaminase (AID) families of cytidine deaminases comprise various members in many species (Harris and Liddament, 2004; Salter et al., 2016; Yang et al., 2017). The APOBEC 
family members can catalyze the deamination of cytidine to uracil in single-stranded nucleic acids, including RNA and single-stranded DNA (ssDNA). By fusing APOBECs with dCas9 or dCas12a proteins, cytosine base editors (CBEs) have been developed to induce $\mathrm{C}$-to- $\mathrm{U}$ deamination in the ssDNA region of the R-loop generated by Cas 9 or Cas $12 \mathrm{a}$ (Hess et al., 2016; Komor et al., 2016; Li et al., 2018d; Ma et al., 2016; Nishida et al., 2016). To enhance editing efficiency, Komor et al. (2016) replaced dCas9 in CBE by nCas9 (D10A), which nicks the target strand and then triggers the endogenous mismatch repair (MMR) pathway (Kunkel and Erie, 2015). MMR resolves the CBE-induced $\mathrm{U} / \mathrm{G}$ mismatch to a U/A pair by removing the unedited Gcontaining strand and then resynthesizes it complementary to the U-containing strand. Finally, the U/A base pair can be converted to a T/A base pair after DNA replication or repair. Although CBEs do not induce DSBs directly, the formation of indels was still found to be triggered by CBE because of the breakage of the abasic site that is formed after the excision of U by uracil DNA glycosylase (UDG) (Lei et al., 2018). Uracil DNA glycosylase inhibitor (UGI) was fused into or co-expressed with CBE to improve purity of the editing product and editing efficiency (Komor et al., 2017; Wang et al., 2017c).

As APOBEC family members can deaminate multiple cytidines in an ssDNA region, all the cytosines in the editing window (a few nucleotides in the spacer region) of CBEs can be edited, which hinders the application of CBEs when single-base editing is required. Thus, various mutations were introduced into the domains involved in the catalytic activity or substrate binding ability of APOBECs to reduce the cytidine deamination activities of CBEs and narrow their editing windows (Kim et al., 2017). In addition, DNA modification or dinucleotide sequence context, e.g., cytosine methylation or $\mathrm{GpC}$ context, also affects the editing efficiencies of CBEs. Hence, naturally occurring APOBECs or in vitro evolved APOBECs, including human APOBEC3A (hA3A) (Wang et al., 2018e) or evoAPOBEC1 (Thuronyi et al., 2019), have been used in CBEs to expand the editing scope.

Alternatively, Escherichia coli tRNA-specific adenosine deaminase (TadA), which catalyzes adenosine to inosine (I) deamination in tRNA, has been engineered to induce adenosine deamination in ssDNA (Gaudelli et al., 2017). Despite the originally low activity, TadA*, which was obtained after seven rounds of directed evolution in vitro, can successfully deaminate adenine in DNA. To further improve the DNA binding activity of TadA*, wild-type TadA was fused at the N-terminus of TadA*; therefore, adenine base editors (ABEs) were developed by fusing nCas9 (D10A) with the TadA-TadA* heterodimer (Gaudelli et al., 2017). As inosines do not exist naturally in DNA, no known DNA glycosylase can efficiently remove inosines from deoxyribose. Thus, no
DNA glycosylase inhibitor is required to be fused into ABEs, and no significant indel formation is triggered by ABEs. Similar to CBEs, subsequent MMR or DNA replication resolves the I/T mismatch to the I/C pair and eventually installs a $\mathrm{G} / \mathrm{C}$ pair at the target site.

Recently, base editors were found to cause OT effects independent of sgRNA or Cas9. CBEs that contain APOBEC cytidine deaminases could induce genome-wide C-to-T/Gto-A mutations at OT sites with no sequence similarity to ontarget sites, suggesting that the OT events are independent of the Cas9/sgRNA targeting module (Jin et al., 2019; Zuo et al., 2019). As APOBEC cytidine deaminases prefer ssDNA regions as deamination substrates, the APOBEC module of $\mathrm{CBE}$ can bind and trigger $\mathrm{C}$-to-U deamination in ssDNA regions generated during various cellular processes, e.g., transcription, DNA replication, and repair (Chen et al., 2019b). Recently, by reducing the substrate-binding/catalytic activity of APOBEC or taking advantage of cytidine deaminase inhibitor domains, sgRNA-independent OT DNA editing was reduced or eliminated (Doman et al., 2020; Jin et al., 2020; Wang et al., 2021; Zuo et al., 2020).

Moreover, APOBEC1, the cytidine deaminase commonly used in base editors, was originally discovered to induce Cto-U editing in apolipoprotein B mRNA, and TadA, which evolved to perform A-to-G DNA editing, is an essential tRNA-specific adenosine deaminase in E. coli. Unexpectedly but not surprisingly, both CBEs and ABEs, which contain APOBEC cytidine deaminases and TadA* adenosine deaminases, respectively, induced transcriptome-wide C-to-U and A-to-I OT mutations (Grünewald et al., 2019a; Zhou et al., 2019a). By engineering the residues of APOBEC or TadA* involved in RNA binding, OT RNA editing was greatly reduced, and on-target DNA editing was maintained (Grünewald et al., 2019b; Zhou et al., 2019a). A recent review also summarized the progress to improve the editing efficiency and precision of CBEs and ABEs (Jeong et al., 2020).

\section{Prime editor}

Although CBEs and ABEs can efficiently induce C-to-U (Gto-A) and A-to-G (T-to-C) transitions, targeted transversions and precise small indels are still hard to generate, as the efficiency of HDR is generally low in most cells and tissues. A versatile gene-editing tool, prime editor (PE), has recently been developed to induce all twelve types of base substitutions, small indels, and their combinations with high efficiency and product purity (Anzalone et al., 2019; Yang et al., 2019). By conjugating nCas9 (H840A) with reverse transcriptase (RTase), the developed PE can initiate reverse transcription (RT) from the single-strand break generated in the nontarget strand under the direction of an engineered prime editing guide RNA (pegRNA). A pegRNA contains 
three parts: a typical sgRNA containing a canonical spacer region for Cas9 recruitment and target-site binding, a primer binding site (PBS) to initiate RT, and an RT template to encode intended edits. Although the original PE induced only low levels of editing in mammalian cells, the editing efficiency was much improved by engineering Moloney murine leukemia virus (M-MLV) RTase to increase the binding ability at the RT initiation site, the thermostability, and the enzyme processivity. To further improve PE efficiency, a canonical sgRNA (nicking sgRNA) was also used to make a nick in the target strand, triggering downstream MMR to remove the unedited strand and maintain the edited strand. Moreover, by using a pair of pegRNAs that contain the same editing information but bind to opposite DNA strands, the efficiency of PE can be improved (Lin et al., 2021).

As the effector module of PE is an RTase from murine retrovirus, a recent study examined whether PE induces genome-wide mutations in plants. At gRNA-dependent OT sites, which have sequence similarity to on-target sites, PE induced minimal OT mutations (Kim et al., 2020). In addition, in contrast to BE, PE induced no observable gRNAindependent OT mutations throughout the genome of plant cells, demonstrating its high editing specificity (Jin et al., 2021).

\section{RNA editing}

In addition to targeting DNA, some class 2 CRISPR/Cas systems can also target RNA. Under the guidance of a single crRNA, Cas13 family members can bind to RNA with a corresponding target sequence, providing a platform for targeted RNA editing (Abudayyeh et al., 2016; Abudayyeh et al., 2017; Konermann et al., 2018; Xu et al., 2021a). Unlike DNA editing, RNA editing does not permanently change genetic information, thus having complementary strengths, e.g., applications in disease treatment without the risk of irreversible side effects. RNA-targeting Cas 13 proteins have been fused with wild-type adenosine deaminase acting on RNA (ADAR) to induce A-to-I base editing in RNA (Cox et al., 2017). Alternatively, ADAR has evolved to deaminate cytidine in RNA and can be used to perform targeted C-to-U RNA base editing (Abudayyeh et al., 2019). Although RNA editing demonstrated considerable efficiency, its OT effects await systematic analysis. Surprisingly, native ADAR can also be recruited by engineered RNAs to perform A-to-I editing at target RNA in the absence of Cas13 protein (Merkle et al., 2019; Qu et al., 2019), which showed low OT effects.

\section{High-throughput functional genomics}

Cutting-edge genome editing technologies enable genomic alterations both individually and in a high-throughput fashion. CRISPR-Cas9 genomic screens drive the latest exciting biological findings, significantly advance the scope and accuracy of functional genomics and have outperformed RNA interference (RNAi) platform, owing to their robustness and scalability (Shalem et al., 2015).

\section{CRISPR screen pipelines and strategies}

Upon Cas9-sgRNA ribonucleoprotein targeting, the RuvC and HNH nuclease domains of Cas9 induce DSBs (Jinek et al., 2012). In response to nuclease-induced DSBs, random indels at the site of DNA cleavage are introduced by the cellular DNA repair system. These indels are vital to generating knockout phenotypes of coding genes.

Selecting active sgRNAs that mediate high CRISPR/Cas9 activity is critical to implement CRISPR genetic screens. The genome-wide selection of sgRNAs can be optimized in a systematic approach; for example, an effective library was created for gene repression and activation screens by applying an algorithm that incorporates chromatin, position, and sequence features (Horlbeck et al., 2016). Multiple sgRNAs targeting the same gene are generally recommended to increase the probability of editing and the robustness of deconvolution. The negative control and nontargeting sgRNAs are critical for quality control of screening and data interpretation. The cell coverage of sgRNAs is another important parameter for library performance.

A robust readout is decisive for a successful screen. The readout can be generalized into two categories (Figure 1). Cells are subjected to universal conditions, as cell growth or death occurs during selection, and the resultant cells can be collected as a whole population. Otherwise, populations of interest have to be enriched by various methods, including biological assays evaluating cell migration, cell attachment, and cellular fluorescence intensity.

Typical examples include screens to identify coding genes conferring resistance to a drug, toxin, pathogen, or immune cells (Guo et al., 2022; Liang et al., 2021; Peng et al., 2015; Ren et al., 2015; Shalem et al., 2014; Zhao et al., 2019; Zhou et al., 2014; Zhu et al., 2021). In a positive screen, the majority of cells are depleted under strong selection conditions, and only a few cells with a protective phenotype expand. In a negative screen, cell growth in a fixed condition and time period can be applied to identify genes whose perturbations lead to cell death or growth inhibition (Shalem et al., 2014; Wang et al., 2014). Fluorescence-activated cell sorting (FACS) is another frequently used assay to enrich or deplete populations of interest in a flexible manner. For example, the surface expression density of a given molecule can be used to enrich cells through FACS. Coding genes regulating immune molecule expression, such as Foxp3, Fas, PDL1, and HLA, have been investigated (Burr et al., 2017; Cortez et al., 2020; 

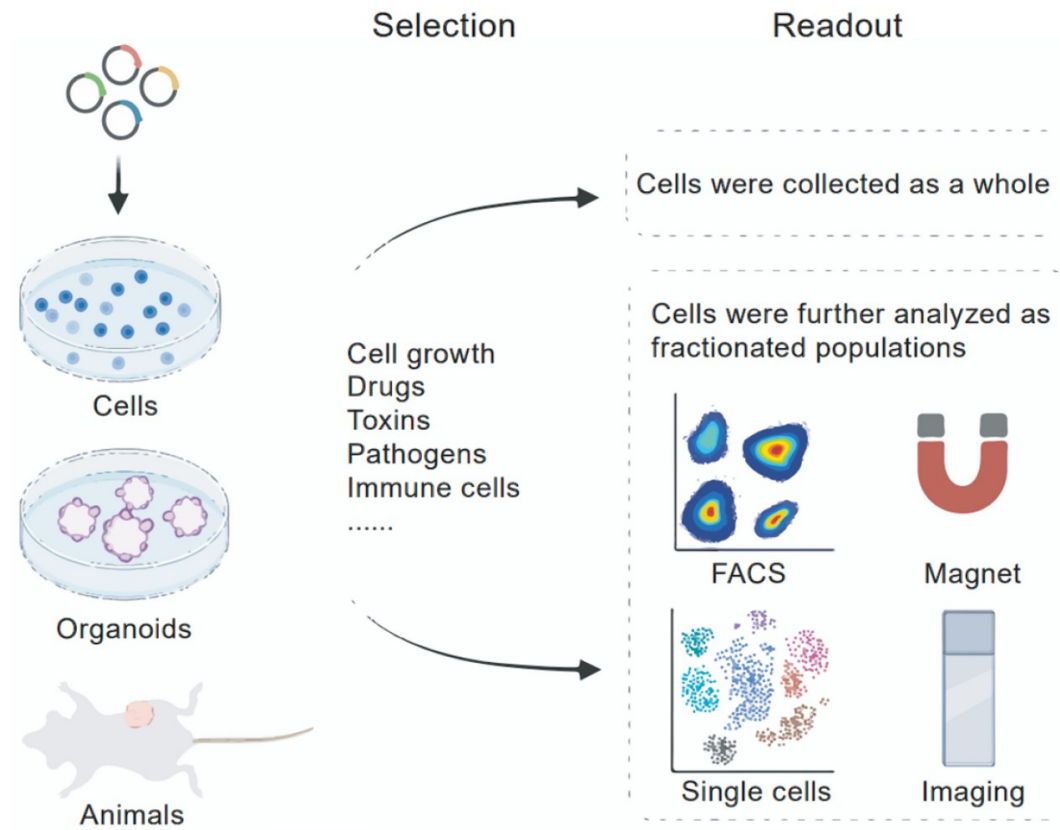

Figure 1 Readouts of CRISPR/Cas9 pooled screens. The readouts can be generalized into two groups. Cells can be collected as a whole for DNA library preparation, or subsets can be further enriched by various methods.

Dersh et al., 2021; Jiang et al., 2019).

Proficient molecular engineers have created Cas9 variants with different nuclease activities, PAM compatibilities, editing windows, and small-molecule or light dependences (Anzalone et al., 2020). Catalytically inactive Cas9 protein (dead Cas9, dCas9) binds a targeted DNA sequence but does not cleave the DNA strands (Pickar-Oliver and Gersbach, 2019). This feature was leveraged to generate Cas9 derivatives by coupling dCas9 with various effectors, such as transcriptional modulators (Gilbert et al., 2013; Qi et al., 2013) or epigenome-modifying factors (Liu et al., 2016). dCas9 transcriptional modulators have been successfully applied to achieve the transcriptional activation (CRISPRa) and repression (CRISPRi) of a gene. They were first established by fusing the transcription activator Vps64 or the Krüppel-associated box (KRAB) repressor to dCas9 (Gilbert et al., 2013). Endeavors have been made to improve the efficacy of these effectors, e.g., CRISPRa effectors were further improved by fusing a repeating peptide array of epitopes to recruit Vps64 (Tanenbaum et al., 2014) or using a synthetic sgRNA scaffold with an MS2 RNA motif loop to recruit additional activators (Joung et al., 2017). These Cas9 derivatives leading to gene transcriptional activation or repression have been employed in functional screens, and the latter type is particularly useful when gene transcriptional perturbation, rather than gene knockout, is preferred. These screens have identified coding genes that modify cell growth and confer cancer therapeutic resistance (Joung et al., 2017; Liu et al., 2016). dCas9-engineered epigenetic effectors have been summarized elsewhere (Nakamura et al., 2021). No- tably, dCas9-coupled gene-regulatory effectors and epigenetic effectors have considerable overlap of gene regulation mechanisms. For instance, transcriptional modulators could shape epigenetic states, and vice versa. Recently, an effector (CRISPRoff) was generated by fusing the transcriptional modulator ZNF10-KRAB and epigenetic effector domains to dCas9 (Nuñez et al., 2021). This effector could silence the specific gene that is stably maintained across cell division and differentiation. All these approaches have demonstrated efficacy in diverse high-throughput genomic screens.

\section{Interrogating noncoding genes}

The CRISPR/Cas9 system offers a general platform for RNA-guided DNA targeting, including coding and noncoding genes. The CRISPR screening approach could be readily extended to systematically discover the functions of numerous noncoding transcripts. MicroRNAs (miRNAs) are small, noncoding RNA molecules that regulate gene expression posttranscriptionally. Small nucleolar RNAs (snoRNAs) constitute a group of intron-encoded noncoding RNAs. CRISPR/Cas9 knockout screens have revealed that multiple miRNAs and snoRNAs regulate cancer cell growth (Cui et al., 2021; Kurata and Lin, 2018; Wallace et al., 2016).

Long noncoding RNAs (lncRNAs) are particularly interesting because of their large numbers and expanding roles in a wide array of cellular processes. Although CRISPR/Cas9 is ideal for inducing frameshift mutations in exons of coding genes to achieve gene knockout, frameshifts are usually not sufficient to disrupt the structure and function of lncRNAs. 
To tackle this, studies have applied large-fragment deletions and splice-site disruption strategies mediated by paired sgRNAs and a single sgRNA, respectively, to disrupt the expression of lncRNAs (Horlbeck et al., 2020; Liu et al., 2018a; Liu et al., 2020; Zhu et al., 2016). These studies have revealed a number of IncRNAs that affect human cancer cell fitness in a cell type-dependent manner. Transcriptional and epigenetic effectors can perturb the expression levels of genes, which is particularly helpful to study the function of lncRNAs. The first CRISPRi screen applied dCas9-KRAB targeting to 16,401 lncRNA loci to evaluate cellular growth in 7 diverse cell lines (Liu et al., 2017a). The same strategy has identified PRANCR as a novel regulator of epidermal homeostasis (Cai et al., 2020). Another study developed a genome-scale CRISPRa screen targeting more than 10,000 lncRNA transcriptional start sites to identify noncoding loci that confer cancer therapeutic resistance (Joung et al., 2017).

Circular RNA (circRNA) is another class of noncoding RNA that features a covalent bond linking the $3^{\prime}$ and $5^{\prime}$ ends generated by backsplicing (Ebbesen et al., 2016). Increasing evidence suggests its role in the regulatory network governing gene expression. Given the identical sequence of circRNAs and their parental mRNA, targeting circRNAforming exons or the intronic cis-elements required for circRNA biogenesis may affect parental gene expression. To specifically perturb the expression of circRNA, a recent study utilized CRISPR-RfxCas13d and gRNAs targeting the RNA sequences spanning back-splicing junction (BSJ) sites. Taking advantage of the low biogenesis efficiencies and turnover rates of circRNAs, this method interferes with circRNA expression more efficiently than linear mRNA and consequently identifies multiple circRNAs contributing to cell proliferation in a cell type-dependent manner ( $\mathrm{Li}$ et al., 2021c).

\section{Mapping regulatory elements}

Proximal and distal enhancers are vital genomic elements dictating gene expression and cell programming. Various biochemical markers and chromatin features are widely used to predict regulatory elements, and reporter assays are applied to assess their effects on gene expression. The CRISPR/Cas9 system now enables researchers to functionally map these elements in a native genomic context through mutation or through transcriptional and epigenetic modulation.

A study was conducted to target predicted enhancer sites of p53 and ER $\alpha$ with CRISPR-Cas in a high-throughput fashion (Korkmaz et al., 2016) and revealed multiple novel functional enhancers. The same strategy was applied to identify CTCF-binding elements (CBEs) that are essential for ER $\alpha$ driven cell proliferation (Korkmaz et al., 2019).

To finely map functional sites in specific enhancers, one study applied tiling libraries to generate saturation mutagenesis in situ at 3 DNase I hypersensitive sites (DHSs) of a BCL11A composite enhancer (Canver et al., 2015). Given that BCL11A abundance is inversely correlated with $\mathrm{HbF}$ protein expression, by the association of $\mathrm{HbF}$ levels and enriched sgRNAs, the study provided a nucleotide resolution map of BCL11A enhancers. The same approach has been applied to map functional sites in regulatory elements of CDKN1A (Korkmaz et al., 2016), NF1, NF2, CUL3, POU5F1 (Diao et al., 2016), Tdgf1, and Zfp42 (Rajagopal et al., 2016). The above studies applied fluorescence tags, surface protein staining, or assay-specific selection reagents to enrich cells of interest. An alternative approach to perform a tiling mutation screen is to apply paired deletion guides in close proximity to genomic loci of interest, including POU5F1 and HPRT1 (Diao et al., 2017; Gasperini et al., 2017).

Base editing strategies apply additional effectors, such as activation-induced cytidine deaminase (AID) (Nishida et al., 2016), APOBEC cytidine deaminases, and TadA adenine deaminase, in combination with the CRISPR-Cas9 geneediting system to introduce point mutations at the targeted site (Gaudelli et al., 2017; Komor et al., 2016). A recent study applied an APOBECB-mediated base editor screen to map regulatory elements of four loci involved in $\mathrm{HbF}$ expression. A total of 6,174 sgRNAs targeting 307 putative regulatory elements were investigated by correlating enriched sgRNAs to $\mathrm{HbF}$ expression abundance, revealing novel therapeutic candidates for sickle cell disease treatment (Cheng et al., 2021).

dCas9 coupled with transcriptional and epigenetic effectors has also been applied to investigate distal regulatory elements. A study applied dCas9-KRAB repressor to assess $\sim 1.3 \mathrm{Mb}$ of genomic sequence surrounding two loci, GATA1 and MYC (Fulco et al., 2016). Multiple distal enhancers contributing to their expression were identified. Another study applied a dCas9-VP64 activator with a tiled library of gRNA targeting sites in the vicinity of CD69 and IL2RA to identify stimulation-responsive enhancers in $\mathrm{T}$ cells ( $\mathrm{Si}$ meonov et al., 2017). Moreover, a study applied dCas9KRAB (CRISPRa) and dCas-9p300 (CRISPRi) as effectors to investigate the enhancers of $\beta$-globin and HER2 (Klann et al., 2017). The above studies demonstrated that CRISPR/ Cas9-based transcriptional and epigenetic screens are successful in identifying genomic regulatory elements.

\section{Probing functional residues}

Tiling libraries generating saturation mutagenesis in situ can also be applied to study functional residues of coding genes. Parsing fragmented DNA Sequences from CRISPR Tiling MUtagenesis Screening (PASTMUS) is a method pairing tiling mutagenesis and NGS to identify functionally critical 
amino acids (Zhang et al., 2019c). Similar tiling mutagenesis using a cytosine base editor to identify functional residues of the protein of interest has also been reported. This method, called CRISPR-X, identified novel mutations that modulate protein expression and confer drug resistance (Hess et al., 2016). Recently, CBE has been applied to probe functional single-nucleotide variants in DDR genes, drug resistance, and cell growth under cellular stresses (Cuella-Martin et al., 2021; Hanna et al., 2021). These studies have demonstrated that base editors are effective in generating single nucleotide polymorphisms (SNPs) at endogenous loci in a highthroughput fashion, suitable for both positive and negative screens.

\section{Exploring genetic interactions}

Given that most human diseases are caused by the combined action of more than one gene, high-throughput screens to illuminate gene interactions are in high demand. With improved dual-gene knockout gRNA vectors (Wong et al., 2016), CRISPR/Cas9-based screens might have been performed in synthetic-lethal studies (Han et al., 2017; Shen et al., 2017). It is still technically challenging to perform unbiased gene-interaction screens simply because of the immense number of pairwise combinations. To date, researchers have mainly focused on druggable and/or tumor inhibitory genes to identify synthetic lethal target pairs (Han et al., 2017; Shen et al., 2017).

Orthogonal CRISPR-Cas9 nucleases from $S$. aureus and $S$. pyogenes have been paired for synthetic lethal screening (Najm et al., 2018), which effectively reduced interference between delivered sgRNAs. Moreover, this combination can be further engineered to carry out a dual screen in which one gene is activated while another is inactivated in the same cell (Boettcher et al., 2018; Zhou and Wei, 2018).

\section{Making better screens}

In the majority of pooled screens, sgRNAs are integrated into the cellular genome for the purpose of decoding by NGS. Thus, libraries are normally introduced by retro- or lentiviral infection at low multiplicity of infection (MOI), usually $<0.3$, to ensure that most cells contain a single integrated sgRNA and thereby minimize the false-positive discovery rate (Shalem et al., 2014; Zhao et al., 2019; Zhou et al., 2014). For genome-wide screens at low MOI infections, a significantly large number of initial cells are required for library construction. A recent study established a strategy that enables infecting cells at a high MOI (Zhu et al., 2019). This assumes that both the false-positive and false-negative rates of screens could be significantly reduced with increasing replicates for each of the gRNAs. By engineering guide RNAs with multiple internal barcodes (iBARs), the enrichment of sgRNAs could be separately assessed with their associated barcodes. This has largely eliminated the codelivery of free riders with functional sgRNAs due to a high-MOI infection, significantly improving screening efficiency and accuracy in positive selection screens. However, the high level of cytotoxicity induced by multiple DSBs in high-MOI library construction hampered the application of the iBAR approach in negative selection screens. To resolve this issue, $\mathrm{Xu}$ et al. (2021c) reported a new genome-wide CRISPR screening method, termed iBARed cytosine base editing-mediated gene KO (BARBEKO). This new screening strategy uses $\mathrm{CBE}$ to generate gene knockout, thus eliminating DSB-related cytotoxicity.

High-throughput functional genomics can be further equipped with other technologies for a flexible readout. CRISPR screens could be combined with single-cell sequencing that profiles transcriptome or chromatin accessibility (Dixit et al., 2016; Jaitin et al., 2016; Rubin et al., 2019). These methods present various advances in detecting indirect or direct indices of sgRNA and linking rich information to single-cell identity (Jaitin et al., 2016; Mimitou et al., 2019; Replogle et al., 2020; Rubin et al., 2019). Combining gene perturbation and phenotypical information offers a great opportunity to tackle the complexity of the biological system, analyze genetic interactions at scale, and dissect gene regulatory networks (Adamson et al., 2016; Norman et al., 2019).

Functional genomic screens in primary cells, organoids, and animals are expected to obtain more physiological insights and relevant therapeutic targets. Although wtCas9based knockout screens are mostly used, DSB-induced cytotoxicity is prone to affect primary cells. A screening strategy to avoid DSBs is to introduce gene knockout by base editors (Billon et al., 2017) or other DSB-independent CRISPR derivatives. BARBEKO has been developed to avoid DSB-related disadvantages in comparison with wtCas9-mediated knockout fitness screens (Xu et al., 2021c). In particular, internal barcodes are integrated into sgRNAs to facilitate high MOI-lentivirus transduction in various types of cells.

For in vivo screens, vectors with improved delivery efficacy, libraries that require fewer primary cells, and effectors that provide more relevant functional insights are in high demand. The in vivo screens can be roughly divided into two conditions: transplantation-based in vivo screening and direct in vivo screening (Chow and Chen, 2018). In transplantationbased screens, sgRNA-containing tumor cells or activated immune cells are transferred to recipient animals and subjected to selection conditions (Chen et al., 2015a; Chen et al., 2021; Dong et al., 2019). Although this approach raises concerns about autochthonous microenvironment representation, the delivery efficacy is relatively high, and delivered sgRNA would not interfere with the rest of the 
cells. Direct in vivo screening is more challenging regarding delivery efficacy, library size, and cell-type specificity. The hydrodynamic injection of sgRNA-containing plasmids can be applied to screen only a handful of genes in hepatocytes using a transposase system (Weber et al., 2015). Chen and colleagues established an in vivo direct screening method to investigate tumor suppressors by delivering a sgRNA library directly into the mouse brain or liver with an AAV vector (Chow et al., 2017; Wang et al., 2018a). These AAV-delivered sgRNAs are unable to integrate into the genome and have to be decoded by targeted-capture sequencing. They further generated AAV-sleeping beauty hybrid vectors for immune cell delivery in vitro, and these vectors can potentially be applied for direct in vivo screens (Ye et al., 2019).

\section{Perspective}

The CRISPR/Cas9 system has revolutionized highthroughput functional genomics. The platform continuously facilitates scientific inquiries and reveals exciting biological findings, including host-microbe interactions, the functionality of immune cells, and cancer treatment candidates. CRISPR/Cas9-based gene perturbations also advance our capacity to study the functions of coding and noncoding genomic elements.

Considering that not all CRISPR/Cas9 tools are compatible with high-throughput purposes, collective efforts to optimize the CRISPR/Cas9 system are highly appreciated. Systematic approaches to optimize the platform would be beneficial to all researchers. Beyond the optimization of libraries and Cas9 effector-related issues, the development of new readouts and friendly pipelines is expected, including bioinformatics-derived insights.

CRISPR/Cas9 knockout screens are widely used and exhibit high consistency between independent screens from different laboratories with similar experimental settings (Goh et al., 2021; Shalem et al., 2014). Despite this encouraging reproducibility, it should be kept in mind that not all significant factors can be identified using one system, relating to the efficacy of guides, the specificity of effectors, heterogeneity of gene expression in different cells, and experimental designs. Thus, applying different Cas9 effectors and experimental settings to study the same scientific question could be complementary to some prior studies.

\section{The application of gene editing in animal model construction}

An increasing number of pathogenic mutations have been identified in the postgenomic era. An animal model paves the way for studying the disease's progression and finding new therapies in medicine. With the development of gene editing tools that can precisely edit target sites, it has become increasingly efficient to generate the desired animals. Hundreds of different kinds of genetically modified animals made with editing tools, especially CRISPR/Cas, have been reported. Here, we review the progress of animal models produced by ZFN, TALEN, and CRISPR/Cas. We will then briefly introduce some established models related to preventing human diseases and discuss the future development of animal models.

\section{Methods to produce the animal model}

RNAi and antisense oligonucleotides (ASOs) can knockdown target gene expression in animals, but neither allows the generation of stable gene knockouts. Homologous recombination (HR) in embryo stem cells is a conventional method to generate gene-editing animals, which requires more than one year to obtain the desired phenotypes. (Dow and Lowe, 2012). Gene-editing technologies significantly improve editing efficiency by more than two orders of magnitude by making DSBs (Rouet et al., 1994). The main process to generate genetically modified animals is to deliver the editing system into the target cells or tissues. For gene knockout via NHEJ, only an editing system was applied. For gene substitution or insertion via HDR, the repair donor should be delivered with the targeting system. The donor may be single-stranded oligodeoxynucleotides (ssODNs) or double-stranded DNA (dsDNA) (Zhang et al., 2021). The editing system could also be used in different formats, including Cas9-sgRNA RNP (Menchaca et al., 2020), RNA (Yang et al., 2013b), virus particles (Swiech et al., 2015), plasmids (Xue et al., 2014), cationic lipid nucleic acids (Zuris et al., 2015), and lipid nanoparticles (Musunuru et al., 2021). Because of the different applications and animal species, there are three main methods to generate genetically modified animals (Figure 2). We will summarize the progress for each method and discuss its advantage and disadvantage.

\section{Embryo microinjection}

Microinjection technology is more than 100 years old. Gene editing using embryo microinjection has been widely applied in many animal species, such as mice (Wang et al., 2013), rats (Ma et al., 2014), monkeys (Niu et al., 2014), pigs (Wang et al., 2015b; Wang et al., 2016; Zhou et al., 2016), goats (Wang et al., 2015a), sheep (Menchaca et al., 2020), zebrafish (Auer et al., 2014) and many other species. All three editing systems, especially CRISPR/Cas9, have been used for embryo microinjection (Martinez-Lage et al., 2017; Sato et al., 2016). The wide application of embryo microinjection is based on editing efficiency. For example, it takes only approximately six months to obtain the desired mouse, regardless of knockout (Li et al., 2013), knock-in (Gurumurthy 


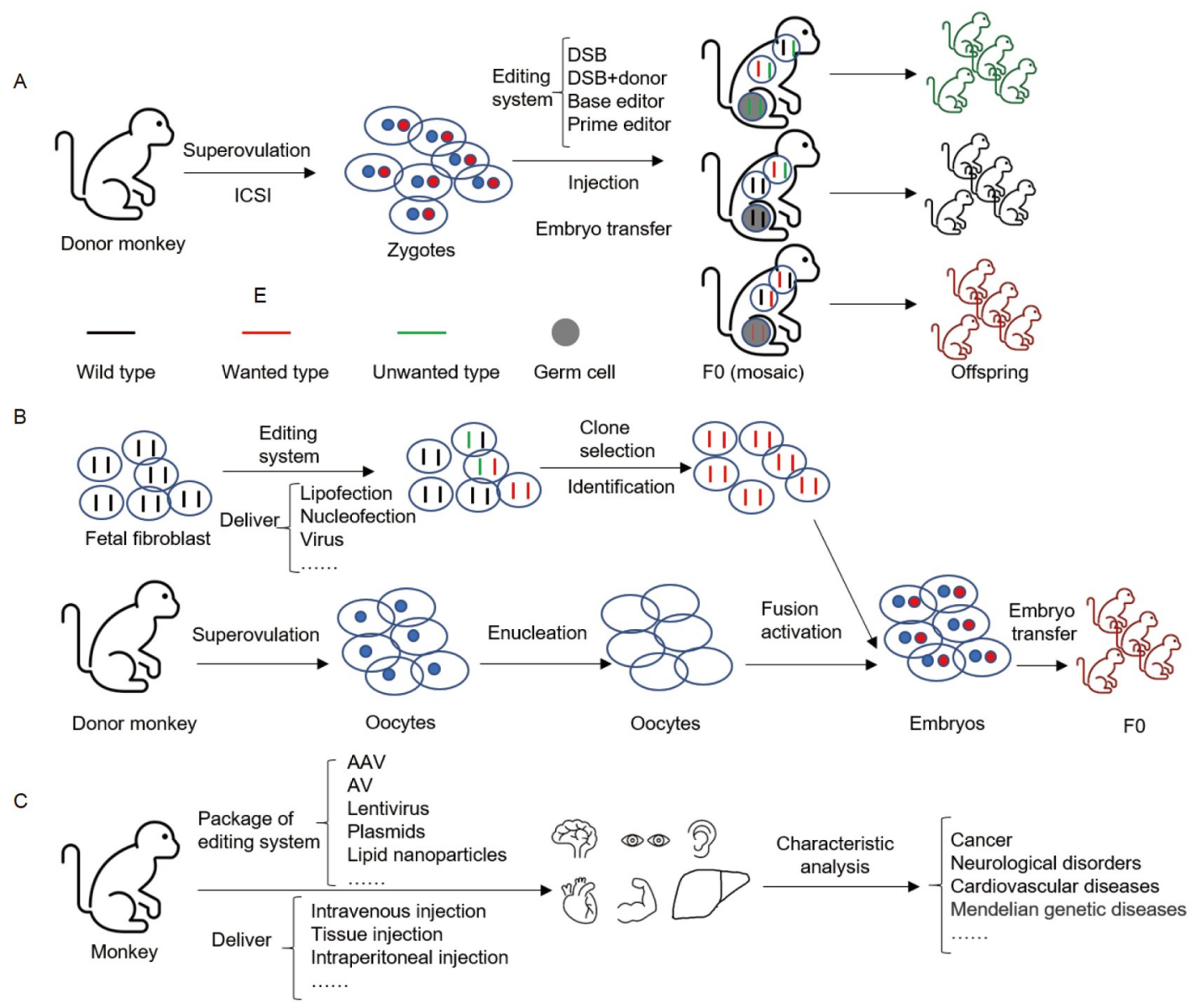

Figure 2 Animal models produced by different methods. There are many different methods to generate animal models. Here, we use monkeys as an example to summarize three main methods. A, Embryo microinjection is widely used to generate animal models. In this method, mosaic animals will be produced in the F0 generation, and only the edited germ cells could pass the desired type to the offspring. For some editing methods, such as the base editing system, the desired animals could be established in the F0 generation. B, Gene editing combined with somatic cell nuclear transfer (SCNT) is another method to generate animal models. The editing system is delivered into fetal fibroblasts, and then the desired cell line is selected. For gene knockin, both positive and negative selection are used. The positive cells are then used for SCNT. For this method, the F0 generation will be the desired animal model. C, For some diseases, such as cancer, in situ injection will satisfy the medical demand. AV, adenovirus.

et al., 2019), or base editing (Ryu et al., 2018). With the development of instruments, it has become more convenient to handle embryo injection with square-wave electroporation, which does not need an experienced operation (Xu, 2019). Because of the heterogeneity of NHEJ, which may result in mosaics, desired animals may be obtained in the F1 generation, especially for gene knock-in. It has been reported that RAD51 delivery facilitates homozygous conversion, which may be applied to generate homozygous animals in F0 in the future (Wilde et al., 2021). For base editing, it is possible to obtain homozygous mutant animals because of the homogeneity and high efficiency of the editing results. Prime editing systems are opening new avenues for point mutation, deletion, and insertion (Anzalone et al., 2019). The improved version may help produce animal models with different kinds of mutations (Liu et al., 2021b). For some species, the embryonic stem cells (ESCs) or induced pluripotent stem cells (iPSCs), edited ESCs or iPSCs could be transferred into the blastula to obtain the desired animals. In such cases, the desired animal model should be produced only when the edited cells are germline cells.

\section{Somatic cell nuclear transfer}

Somatic cell nuclear transfer (SCNT) provides another method to produce animals with a consistent genetic background. SCNT has been applied in more than 20 different species, including pigs, mice, monkeys (Liu et al., 2018b), and others. For gene editing animal models using SCNT, fetal fibroblasts are used to receive the editing system, and then the positive cell clone will be picked and used as the donor for SCNT. Pigs and monkeys are two important model animals in human medicine. Gene editing combined with SCNT has been achieved in both species (Liu et al., 2018b; Yan et al., 2018; Zhou et al., 2015). The advantage of SCNT is that the animals produced by this technology are consistent without mosaics (Ryczek et al., 2021). For large animals, 
gene editing combined with SCNT has an advantage compared with embryo microinjection. The disadvantage of SCNT is abnormal development and poor efficiency. Some effective improvements will be useful for its application (Czernik et al., 2019).

\section{In situ injection}

The animal models produced by embryo microinjection and SCNT have modified genes in all tissues. For conditional editing animal models, the Cre model could be used, and it will take a long time to observe the phenotype. For some human diseases, such as cancer, tumor cells initiate in some tissues, not the whole body. In situ injection showed an advantage for this purpose. Gene editing in adult animals could be used to simulate the development of tumor cells (Zuckermann et al., 2015). Recently, different methods have been reported to study the tissue-specific functions of genes. One of them is to establish a model with Cas9 expression in a specific tissue, and then the sgRNA could be introduced into animals (Carroll et al., 2016). Another method is to inject Cas9 and sgRNA simultaneously (Zuckermann et al., 2015). The injection methods and the package of the editing system vary. Tail intravenous injection, tissue injection (Zuckermann et al., 2015; Zuris et al., 2015), and intraperitoneal injection (Carroll et al., 2016) have all been reported. There are several options for the delivery of the editing symptoms, such as adeno-associated virus (Lin et al., 2020), adenovirus (Ding et al., 2014a), lipid nanoparticles (Musunuru et al., 2021), plasmid (Xue et al., 2014), and cationic lipid nucleic acid (Zuris et al., 2015). Furthermore, to accurately control gene expression after injection, some inducible elements are used, such as light (Konermann et al., 2013) and drugs (Dow et al., 2015). Somatic gene editing through tissue injection has been applied in several kinds of cancer (Maresch et al., 2016; Sánchez-Rivera et al., 2014; Zuckermann et al., 2015). Compared to embryo microinjection and SCNT, tissue injection requires less time to observe the desired phenotype, although the editing efficiency of this method should be improved.

\section{Animal models promote mechanistic studies and treatment of human diseases}

Gene editing technology greatly speeds up the production of animal models, which help to interrogate human diseases. Here, we summarize the progress on animal models depending on the disease categories. There are also animals that are used as tools for producing specific models, such as Cas9-expressing pigs (Wang et al., 2017b). Instead of listing all reported animal models made of editing technology, we focus on some representative cases of human diseases, especially large animals, e.g., nonhuman primates (Chen et al., 2016) and pigs (Ladowski et al., 2019).
Cancer

Cancer, as one of the most detrimental diseases involving multiple processes, is responsible for the loss of tremendous amounts of life every year. Suitable animal models may help to recapitulate the underlying mechanism of cancer development (Mao et al., 2016; Torres-Ruiz and Rodriguez-Perales, 2015). The gene-editing system makes it possible to generate multiple gene mutations and paves the way to interrogate the development and progression of cancers. Some animal models have been established to target the genes related to given cancers. Xue et al. (2014) used hydrodynamic injection to deliver a CRISPR plasmid to the liver in mice. Three genes, Pten, p53, and CTNNB1, were targeted. The editing efficiency for Pten was approximately $2.6 \% \pm 1.4 \%$. They found that a mutation of Pten would elevate Akt phosphorylation and lipid accumulation in hepatocytes. Platt et al. (2014) demonstrated that targeting KRAS, p53, and $L K B 1$ in Cre-dependent Cas9 knock-in mice would lead to macroscopic tumors of adenocarcinoma pathology in lung adenocarcinoma. They used AAV as a donor to generate the $K R A S^{\mathrm{G} 12 \mathrm{D}}$ mutation, and the efficiency was approximately $1.8 \%$ at nine weeks postdelivery. Maresch et al. (2016) delivered CRISPR/Cas9 targeting multiple genes to the pancreas of adult mice, and they observed that $54 \%$ of the mice developed pancreatic cancer within 24 weeks. There will be large animal models and potential target genes available in the future. Thus far, mice and zebrafish are the two main species that have been leveraged to produce models for cancer research ( $\mathrm{Li}$ et al., 2021d). There will be more large animal studies and novel genes to be targeted in the future.

\section{Neurological disorders}

Neurological disorders are the leading cause of disability and greatly compromise the quality of life, such as Alzheimer's disease, Huntington's disease, Parkinson's disease, amyotrophic lateral sclerosis, and stroke (Choong et al., 2016). There are many mouse models for neurological disorders (Swiech et al., 2015; Tsuchiya et al., 2015). Because of the difference between human and rodent models, most neuroprotective therapies fail at the stage of translation despite the fact that the animal model plays a vital role in studying such diseases (Chesselet and Carmichael, 2012). The wide use of gene editing systems makes it convenient to simulate gene mutations in large animals, such as monkeys (Kang et al., 2019). Large animals may be more suitable for neurological disorders. Zhou et al. (2019b) generated the SHANK3-mutant macaques. The sgRNA targeted exon 21 of the SHANK3 gene, and the editing system was microinjected into the embryos. Finally, they obtained five live newborns, 2 of them did not have the wild-type genotype. The correct phenotype of animals exhibited sleep disturbances, motor deficits, and increased repetitive behaviors, which is consistent with autism 
spectrum disorder. Chen et al. reported that they generated TALEN-edited MECP2 mutant cynomolgus monkeys. They microinjected TALEN-RNA into embryos and obtained five female mutants. After a comprehensive analysis of the mutant monkeys, they found a series of physiological, behavioral, and structural abnormalities resembling clinical manifestations of Rett syndromes (Chen et al., 2017b; Novarino, 2017). Yan et al. (2018) used CRISPR/ Cas9 and SCNT to establish a knockin pig model that expresses full-length mutant huntingtin. They transfected fetal pig fibroblast cells with Cas9, sgRNA and the donor. A total of 2,430 fetal pig fibroblast cell clones were picked, and 9 of them contained heterozygously expanded human HTT exon 1. One of these was chosen for SCNT. The mutant pigs showed consistent movement, behavioral abnormalities, and early death, which conformed to the phenotype of Huntington's disease. Gene editing and SCNT are widely used in large animal models to avoid mosaics and heterogeneity (Cibelli and Gurdon, 2018; Cyranoski, 2018).

\section{Cardiovascular disease}

Cardiovascular disease (CVD) is the first-largest disease in terms of morbidity and mortality. There are several types of cardiovascular disease that are the leading causes of morbidity and mortality (Savoji et al., 2019). There is an urgent need to develop animal models for mechanistic studies and therapeutic evaluation. Many genes or single nucleotide polymorphisms are related to CVD, such as PCSK9 and $A P O E$ (Heianza and Qi, 2019). Animal models targeting different genes have been established (Carreras et al., 2019; Carroll et al., 2016; Chadwick et al., 2017; Ding et al., 2014a; Musunuru et al., 2021; Wang et al., 2018d; Yuan et al., 2018). Ding et al. (2014a) used adenovirus to deliver a CRISPR/ Cas9 system targeting the Pcsk9 gene in mouse liver. The editing efficiency of $P \operatorname{csk} 9$ was as high as $>50 \%$. They found that the mouse had decreased plasma levels of PCSK9. Carreras et al. (2019) generated a liver-specific human PCSK9 knock-in mouse model. The human PCSK9 gene was inserted at the Rosa 26 locus. The positive ES clone was picked using neo and DTA elements. The mutant model showed a human-like hypercholesterolemia phenotype. Musunuru et al. (2021) used the base editor in living cynomolgus monkeys to target the PCSK9 gene and demonstrated that the monkeys had reduced blood levels of PCSK9 and low-density lipoprotein cholesterol. Carroll et al. (2016) generated cardiac-specific Cas9 transgenic mice and then used adeno-associated virus 9 to target Myh6. The mice displayed severe cardiomyopathy and loss of cardiac function. Considering the complexity of CVD, an increasing number of animal models that target different genes should be produced. Animal models will benefit drug screening (Savoji et al., 2019).

\section{Xenotransplantation}

Xenotransplantation provides an alternative method for alleviating the shortage of organs for human transplantation. Most recent xenotransplantation research has focused on genetically modified pigs. Niu et al. (2017) used CRISPR/ Cas9 to inactive all the porcine endogenous retroviruses in pigs. The cell surface carbohydrate antigens in pigs preclude the success of porcine xenografts. Many studies have focused on the elimination of the surface carbohydrate antigens (Butler et al., 2016; Fischer et al., 2020; Fu et al., 2020; Ladowski et al., 2019; Ryczek et al., 2021). In the future, the "ideal" modified pig could be produced with advanced geneediting technology for the benefit of patients.

\section{Regeneration}

Regeneration means the ability to replace or restore the injured body. The success of regeneration in mammals will greatly benefit patients. The mechanism of regeneration remains to be elucidated. Lizards, salamanders, and zebrafish show the ability to regenerate (Daponte et al., 2021). Modified animal models should be generated to uncover the mechanism of regeneration. Recently, genetically modified lizard and zebrafish have been reported (Auer et al., 2014; Rasys et al., 2019). In the future, with the help of gene editing, animal models may help to achieve regeneration in mammals.

\section{Development}

Some gene mutations result in aberrant development, such as adrenal hypoplasia congenita (AHC), which is caused by a $D A X 1$ mutation. Kang et al. generated the $D A X 1$ mutation in cynomolgus monkeys. These $D A X 1$-deficient monkeys showed defects in adrenal gland development and abnormal testis architecture with small cords. The observed phenotype resembles the findings in human patients, demonstrating that the animal models were suitable for AHC. The SIRT6 gene encodes a longevity protein in rodents, and its function in primates remains unknown. Zhang et al. (2018c) produced the SIRT6 knockout in cynomolgus monkeys, which showed developmental retardation. These results may provide mechanistic insight into human perinatal lethality syndrome. African turquoise killifish is a naturally short-lived vertebrate and a good model to study aging. Harel et al. (2015) reported a platform that could generate genetically modified killifish for aging research. They acquired Tert mutant killifish using CRISPR/Cas9 and found that $\operatorname{Tert}^{48 / \Delta 8}$ fish exhibited a progressive loss of fertility in the first generation.

\section{Other Mendelian genetic diseases}

There are many other disease-related animal models. Huang et al. (2019) produced HBB-deficient Macaca fascicularis monkeys manifesting severe $\beta$-thalassemia phenotypes, 
which could be a valuable model for studying the mechanism of $\beta$-thalassemia and evaluating therapeutic interventions and drug effects. Chen et al. (2015b) reported the disruption of the dystrophin gene in rhesus monkeys and found that the mutant monkeys presented markedly depleted dystrophin and muscle degeneration. Lin et al. (2020) generated a nonhuman primate model by subretinal delivery of an AAV-mediated CRISPR-Cas9 system targeting $C N G B 3$. The editing efficiency was $12 \%-14 \%$. They observed a reduced response of electroretinogram in the central retina. Menchaca et al. (2020) produced a sheep model with mutations in the Otof gene. They used CRISPR/ Cas9 combined with an ssODN to generate mutant lambs. A total of $17.8 \%$ of the lambs showed indels, and $61.5 \%$ of the edited lambs carried knock-in mutations. Large animals with disease-relevant mutation(s) may pave the way to treat malfunctions and facilitate successful translation from animals to humans.

\section{Perspective}

To date, mice are the most popular animals for simulating human diseases. Genome editing technology is opening a new avenue for efficiently producing animal models (Martinez-Lage et al., 2017). Considering the complexity of human diseases, it is pivotal to build suitable animal models. Recently, most genetically modified animals have been produced by knocking out target genes. In the future, animal models not only provide tools to understand disease mechanisms but are also valuable tools to evaluate therapeutics and clinical interventions, such as large animals and humanized animals.

Some characteristics of large animals, such as neuroanatomical, physiological, perceptual, and behavioral characteristics, are relatively similar to those of humans (Izpisua Belmonte et al., 2015). Large animals, such as chimpanzees, monkeys, pigs, and dogs, not only provide materials to accurately dissect disease mechanisms but are also suitable models to cure diseases, especially in the brain.

Previously reported animal models were produced mainly via gene knockout or point mutations. Base editing system and prime editing could efficiently achieve the base transition, transversion, insertion or deletion of a small fragment. It is still difficult to precisely handle large fragments. Few animals were obtained by replacement or knocking in the large fragment. Although some methods have been reported to insert large fragments, such as the INTEGRATE system (Vo et al., 2021), transposon-directed integration (Klompe et al., 2019; Strecker et al., 2019b) and CRISPRRetron system (Sharon et al., 2018), their applications in mammals remain to be tested. The efficient generation of humanized animals, especially large animals, will be a future direction.

\section{Diagnosis}

\section{The development of CRISPR diagnosis}

In addition to its application in genome editing, CRISPR technology has recently been repurposed for the detection of target nucleic acids, among other tasks (Leung et al., 2021; $\mathrm{Li}$ et al., 2019b). Compared with traditional molecular diagnostic methods such as polymerase chain reaction (PCR), CRISPR-based diagnostic methods have shown many advantages in rapidness, accuracy, and portability and have been called next-generation molecular diagnostics (MDx) (Chertow, 2018). Based on the catalytic principles of the employed Cas proteins, the recently developed CRISPR diagnosis (CRISPR-Dx) systems can be simply classified into two categories (Figure 3). The first category employs Cas9 to detect target nucleic acids with the help of its high specificity in nucleic acid recognition. The second category uses Cas proteins with trans-cleavage activities, such as Cas 12 and Cas13, and shows high specificity and sensitivity in detecting both nucleic acids and other molecules of interest ( $\mathrm{Li}$ et al., 2019b).

\section{Cas9-based CRISPR-Dx}

The utilization of Cas9 and its mutants in nucleic acid detection undoubtedly constituted the prologue of CRISPR-Dx (Deng et al., 2015; Pardee et al., 2016). With the combination of an isothermal RNA amplification method called NASBA (nucleic acid sequence-based amplification), Cas9 was successfully used for pathogen diagnostics (Pardee et al., 2016). The obtained method, namely, NASBACC (NASBACRISPR Cleavage), takes advantage of Cas9-mediated precise cleavage and discriminates between distinct viral strains with single-base resolution. Specifically, target RNA is first amplified by NASBA, and the obtained amplicons are then digested with Cas9. If the target sequence perfectly matches the sgRNA, it will be cleaved by Cas9 and unable to trigger the coupled toehold RNA sensors. However, if the target sequence contains a mutation that prevents Cas9 cleavage, the intact amplicons are then transcribed to RNA sequences to switch on the sensor, resulting in a visible color change in the paper disc (Pardee et al., 2016). Moreover, to achieve high detection specificity in strain discrimination, strainspecific PAM sites can be employed for designing sgRNAs.

Since then, several CRISPR-Dx systems have been created by integrating Cas9 with different types of nucleic acid amplification methods, including nicking endonucleasemediated nucleic acid amplification in CAS-EXPAR (Huang et al., 2018), rolling circle amplification (RCA) in CasPLA (Zhang et al., 2018b), RACE (Wang et al., 2020d), and the PCR method ctPCR (Wang et al., 2018c) and CARP (Zhang et al., 2018a). These methods mainly use Cas9-mediated precise cleavage activities and detect either fluorescent signals or amplicon sizes, all of which have shown both 


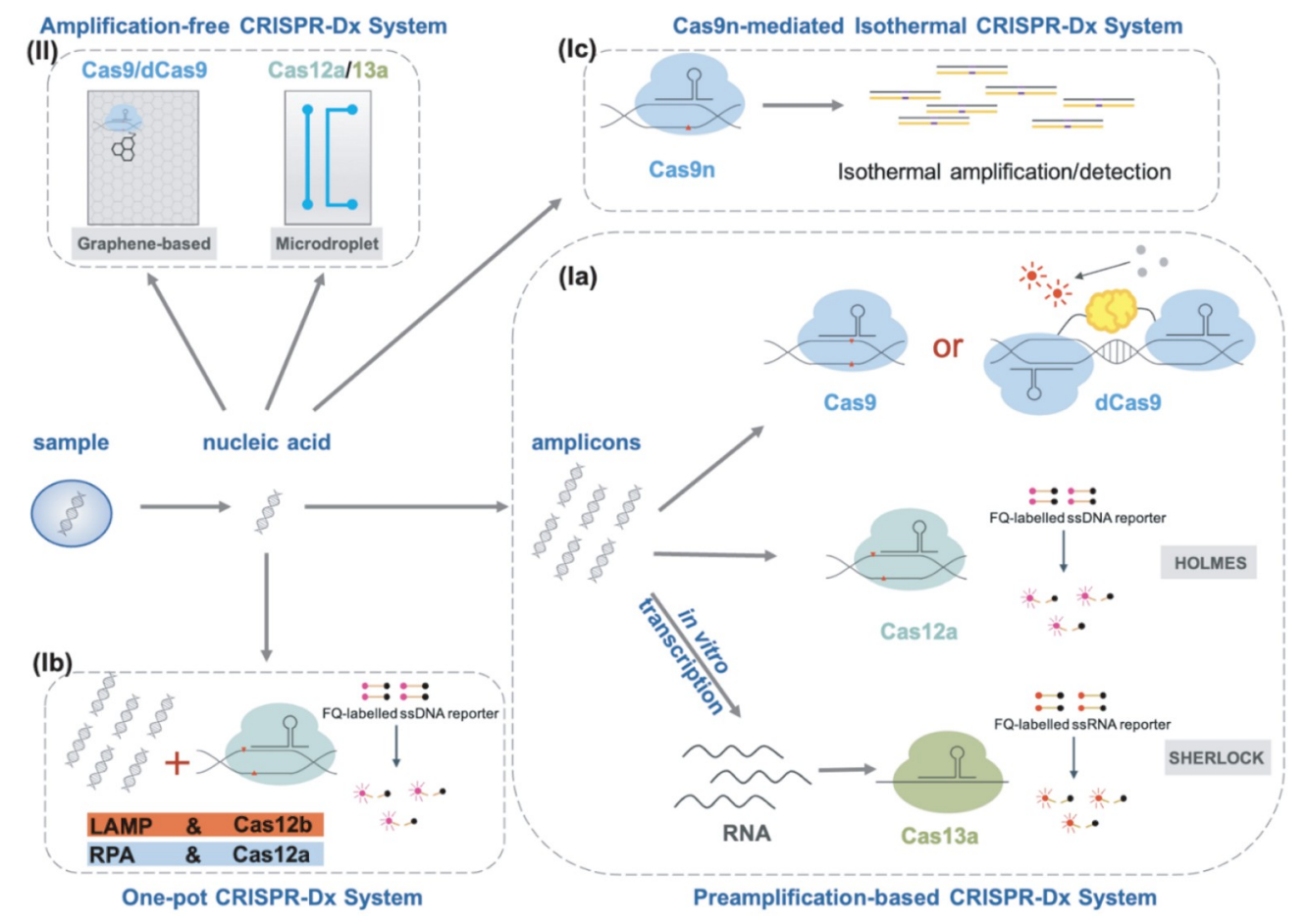

Figure 3 CRISPR-based diagnostic systems for nucleic acid detection. Nucleic acid is first released from samples via direct lysis or nucleic acid extraction procedures and detected by distinct CRISPR-Dx methods. Briefly, the CRISPR-Dx methods can be classified into two classes, including (I) amplificationdependent and (II) amplification-free methods. (Ia) Target nucleic acids are first preamplified by either PCR or isothermal amplification methods, and the amplicons are then detected by (d)Cas9-based or trans-cleavage-based next-generation CRISPR-Dx methods. (Ib) With the integration of isothermal amplification and trans-cleavage reactions, target nucleic acids can be detected in a one-pot system. Usually, Cas12a is combined with (RT-)RPA, while Cas12b and thermophilic (RT-)LAMP can be placed in one pot. (Ic) Cas9n is combined with isothermal amplification systems or Klenow polymerase to achieve accurate and isothermal nucleic acid detection. (II) CRISPR-Dx systems for amplification-free nucleic acid detection.

sensitivity and specificity in nucleic acid detection.

Another large group of detection methods employs dCas9, which has lost its nuclease activity, to specifically recognize and bind target nucleic acids. For example, the first CRISPRbased nucleic acid detection system, termed CASFISH (Cas9-mediated fluorescence in situ hybridization), employed fluorescently labeled dCas 9 and sgRNA to precisely target and visualize genomic loci, revealing their great potential in genetic diagnosis (Deng et al., 2015). Recently, with the employment of sgRNA linked with tandem MS2 aptamers and dCas9, a similar method, namely, RCasFISH (CRISPR/dCas9-MS2-based RNA fluorescence in situ hybridization assay), was developed to image and quantitate RNA transcripts at a single-molecule level (Wang et al., 2020c). Similar to Cas9, target nucleic acids can be preamplified to enhance the dCas9-based detection sensitivity. In addition to the fluorescently labeled probes, the coupled reporter can be split into two half domains, each half fused with an intact dCas9, and only when a pair of dCas9 binds to the target sequences in proximity can the split halves reconstitute an active reporter to produce signals. This strategy has been successfully employed in the development of PCR reporters (Zhang et al., 2017a) and RCH (Qiu et al., 2018), both of which are of high specificity due to the coexistence of two sgRNA binding sites as a prerequisite.

Scientists also use a Cas9 nickase (Cas9n) with only the functional RuvC domain to develop isothermal amplification systems for nucleic acid detection. For example, the CRISRDA method integrates Cas9n with the strand displacement amplification (SDA) method (Zhou et al., 2018), while the Cas9nAR method merely uses two enzymes of Cas9n and the Klenow polymerase (exo-) (Wang et al., 2019 c), both of which exhibit high sensitivity and singlebase resolution.

Other systems, such as CASLFA (Wang et al., 2020e), may use either Cas9 or dCas9 for the specific recognition of target sequences. CASLFA first uses either PCR or isothermal amplification for nucleic acid preamplification and takes advantage of the lateral flow assay (LFA) for convenient signal detection. Another promising CRISPR-Dx strategy is to apply a graphene-based field-effect transistor, which anchors the dCas9-sgRNA complex (dRNP) to a graphene monolayer and detects the on-chip electrical response after dRNP binds the target nucleic acid sequences. Notably, this method (namely, CRISPR-Chip) directly detects target sequences within $15 \mathrm{~min}$, with a sensitivity of $1.7 \mathrm{fmol} \mathrm{L}^{-1}$, and is amplification-free (Hajian et al., 2019). On the basis of CRISPR-Chip, the SNP-Chip is then created with the 
employment of either Cas9 or dCas9 and discriminates between homozygous and heterozygous DNA sequences from unamplified patient samples (Balderston et al., 2021), demonstrating its great potential in diagnosis.

\section{Cas12-based CRISPR-Dx}

Cas9-based CRISPR-Dx technology has provided a new method for rapid nucleic acid detection. In addition, the discovery of the trans-cleavage activities of some Cas proteins has accelerated the development and application of CRISPR-Dx technologies, introducing the era of next-generation MDx. Usually, a Cas protein recognizes target nucleic acid sequences with the guidance of crRNA and cleaves the target sequences at specific sites, which is called ciscleavage (Li et al., 2018b). While some Cas proteins exhibit nonspecific cleavage activities against nontarget singlestranded nucleic acids, these activities are designated transor collateral cleavage activities (Abudayyeh et al., 2016; Li et al., 2018b). To date, two types of Cas proteins have been demonstrated to possess trans-cleavage activities, including the type V Cas12-family and the type VI Cas13-family proteins (Li et al., 2019b).

The Cas 12 protein recognizes and cis-cleaves the target dsDNA or ssDNA under the guidance of crRNA or sgRNA (Yang et al., 2016; Zetsche et al., 2015). Several Cas12 family members have been characterized to possess transcleavage activities, including Cas $12 \mathrm{a}$ (previously called Cpf1), Cas12b (previously called C2c1), and Cas12F (previously called Cas14) (Chen et al., 2018; Harrington et al., 2018; Li et al., 2018b; Li et al., 2019a), and have been employed to develop diverse CRISPR-Dx systems. The transcleavage activities of Cas12a were first identified during the exploration of its cleavage activity against target ssDNA, and researchers found that once a ternary complex of Cas12a, crRNA and target dsDNA (or ssDNA) was formed, Cas12a was triggered to randomly cleave nontarget ssDNA in the system (Li et al., 2018b). Therefore, Cas12a has the properties of both target-specific binding and nonspecific ssDNA trans-cleavage and was used to create the CRISPR-Dx method, namely, HOLMES (a one-hour low-cost multipurpose highly efficient system) (Li et al., 2018c; Li et al., 2019b), which exhibits attomolar detection sensitivity and single-base resolution. Another group independently developed a similar Cas12a-based diagnostic method named DETECTR (Chen et al., 2018). Both methods employ Cas12a trans-cleavage activities to randomly cleave the fluorophore quencher (FQ)-labeled ssDNA reporter, and the only difference between them is that different preamplification methods are applied, i.e., PCR in HOLMES and RPA in DETECTR. Although the two methods were published almost at the same time, HOLMES was patented first and had a much earlier priority date than DETECTR (Wang et al., 2017a).
On the basis of HOLMES (or DETECTR), dozens of methods have recently been developed to further improve the diagnostic performance and practicability, including sample processing (Joung et al., 2020; Ning et al., 2021), target nucleic acid amplification (Aman et al., 2020; Ding et al., 2020; Lee et al., 2020), CRISPR reaction (Nguyen et al., 2020; Yue et al., 2021) and signal detection (Dai et al., 2019; Huang et al., 2020a; Nouri et al., 2020; Shao et al., 2019; Tao et al., 2020; Zhang et al., 2020). For example, nucleic acids can be released from direct sample lysis with an optimized extraction solution and concentrated by magnetic beads, eliminating tedious nucleic acid purification steps (Joung et al., 2020; Ning et al., 2021). In the original procedure of either HOLMES or DETECTR, the steps of nucleic acid amplification and CRISPR trans-cleavage are separated. Thus, a standard PCR amplification laboratory with independent areas of amplification and analysis rooms is required to prevent aerosol contamination while uncapping the amplification tubes and transferring the amplicons. To make the diagnosis more portable, Cas12a is then integrated with isothermal amplification methods such as RPA to create a one-pot detection system (Nguyen et al., 2020; Yue et al., 2021). The CRISPR reaction conditions have also been optimized, including the reaction buffer and the crRNA sequences, to further improve the cleavage efficiencies (Nguyen et al., 2020; Yue et al., 2021). In addition to fluorescent ssDNA reporters, different types of labeling and detection have been explored. For example, reporters can be dual labeled with FAM and biotin, and CRISPR-Dx can be combined with LFA technology (Bai et al., 2019; Broughton et al., 2020; Lu et al., 2020; Mukama et al., 2020) to facilitate the simple observation of diagnostic results on test strips without the requirement of ancillary instruments. Alternatively, the Cas12a-based trans-cleavage reaction can be integrated with cascade enzymatic and Fenton reactions, leading to a visible color change to indicate the diagnostic results (Huang et al., 2020a). Similar to the Cas9-based CRISPR-Chip technology that detects electrical outputs, the Cas12a-mediated trans-cleavage signals can also be read by electrochemical responses (Dai et al., 2019; Zhang et al., 2020) or solid-state nanopore sensors (Nouri et al., 2020), both of which can improve the detection sensitivities and reduce the detection time.

Cas $12 \mathrm{~b}$ is a type- $\mathrm{Vb}$ thermophilic Cas protein and exhibits trans-cleavage activities at higher temperatures (e.g., above $55^{\circ} \mathrm{C}$ ). It can be combined with high-temperature isothermal reactions such as LAMP (loop-mediated isothermal amplification). With the use of Alicyclobacillus acidoterrestris Cas12b (AacCas12b) and LAMP, the first one-pot CRISPRDx system, namely, HOLMESv2, was developed, which markedly reduces the risk of aerosol contamination (Li et al., 2019a).

Cas12F, which is of type $\mathrm{VF}$, also has trans-cleavage 
activities; however, unlike Cas 12a and Cas $12 \mathrm{~b}$, it recognizes only ssDNA. Therefore, the Cas $12 \mathrm{~F}-$ based method, namely, Cas14-DETECTR, requires a step to transform dsDNA amplicons to ssDNA before Cas12F-mediated targeting. In addition, compared to Cas12a and Cas12b, Cas12F has less efficient trans-cleavage activities, and a much longer reaction time is required, which may limit the breadth of its application in diagnosis (Harrington et al., 2018).

\section{Cas13-based CRISPR-Dx}

The Cas13-family proteins instead recognize target singlestranded RNA (ssRNA) and trans-cleave collateral ssRNA sequences in the reaction system (Abudayyeh et al., 2016). With the combination of Cas13a and RPA isothermal amplification, the first CRISPR-Dx method using Cas13 transcleavage activities, namely, SHERLOCK (Specific High Sensitivity Enzymatic Reporter UnLOCKing), was developed, exhibiting attomolar sensitivity and single-base mismatch specificity (Gootenberg et al., 2017). Because of the nonspecific properties of the trans-cleavage reactions, HOLMES and SHERLOCK are unable to detect multiple targets in one reaction, which limits the clinical applications of the CRISPR-Dx tools because an internal control is usually required in such scenarios. The multiplexed SHERLOCKv2 method was developed through the combination of Cas13 and Cas12a to solve this problem (Gootenberg et al., 2018). Different Cas13 orthologs have distinct cleavage preferences for dinucleotide RNA reporters, reflecting the orthogonality between Cas 13 enzymes and enabling the detection of multiple targets in one pot. As Cas12 is orthogonal to Cas13, the multiplexity was further increased by the addition of Cas12a, achieving 4-channel multiplexing in a single SHERLOCKv2 reaction. In addition, SHERLOCKv2 successfully detects Dengue or Zika viruses and their mutants via LFA strips, which further highlights its potential as a multiplexable and portable nucleic acid detection system. Moreover, on the basis of SHERLOCK, dozens of methods have been recently developed and used in different scenarios, including COVID-19 diagnosis (Ackerman et al., 2020; Fozouni et al., 2021; Lee et al., 2020; Myhrvold et al., 2018; Patchsung et al., 2020; Shinoda et al., 2021; Zhou et al., 2021).

\section{CRISPR-based non-nucleic acid detection}

In addition to targeting nucleic acid diagnosis, CRISPR-Dx can also be used for the detection of non-nucleic acid (NNA) targets such as small molecules (Liang et al., 2019), metal ions and sodium ions (Xiong et al., 2020), proteins (Liu et al., 2021a), extracellular vesicles (EVs) (Zhao et al., 2020a; Zhao et al., 2020b) and cells (Li et al., 2021a). During NNA detection, NNA targets first lead to the generation of target nucleic acids that can be recognized by Cas proteins and trigger trans-cleavage reactions, which actually employ CRISPR-Dx as a signal amplifier. For example, in combination with allosteric transcription factors (aTFs), Cas12a can be used to rapidly detect small molecules from clinical samples (Liang et al., 2019). Specifically, target small molecules release aTFs from binding to dsDNA sequences, which serve as the target of Cas12a activator to trigger Cas12a-mediated trans-cleavage reactions.

In addition to aTFs, functional nucleic acid aptamers can be combined with Cas proteins to detect specific NNA targets, using both the high affinity and specificity of aptamers and high trans-cleavage activities of Cas proteins. Usually, the recognition and binding of NNA targets such as proteins or small molecules can be induced to change the conformation of aptamers, which then serve as Cas $12 \mathrm{a}$ activators to produce trans-cleavage signals. In a recent study, allosteric aptamers were employed to recognize and bind the penicillin-binding protein $2 \mathrm{a}(\mathrm{PBP} 2 \mathrm{a})$ protein on the surface of methicillin-resistant Staphylococcus aureus (MRSA), which resulted in the release of the initiator sequence to subsequently unfold a second hairpin probe to serve as the Cas12a activator. The introduction of Taq DNA polymerase and $\mathrm{Nb}$.BbvCI nicking enzyme allows the continuous generation of Cas12a activators and enhances the detection sensitivity (Wei, 2021).

Similar strategies have been developed to detect target proteins on the surfaces of other bacteria, such as Acinetobacter baumannii (Li et al., 2020a) and tumor-derived EVs (Li et al., 2021b). After the binding of target proteins, aptamers can produce templates for in vitro transcription to generate crRNA and then initiate the Cas12a trans-cleavage reaction in a system that lacks crRNA (Zhao et al., 2020a). When aptamers are combined with immunoassays, one aptamer sequence is immobilized on the plate substrate to capture target proteins, and the other aptamer sequence then recognizes distinct structural conformations of the same target and links the Cas12a ternary complex to produce trans-cleavage signals (Li et al., 2021a). In addition, an aptamer-based CRISPR-Dx system can detect small organic molecules and metal ions, e.g., ATP and $\mathrm{Na}^{+}$ions (Li et al., 2021a; Xiong et al., 2020), similar to protein detection. In combination with hybridization chain reaction (HCR), aptamers may bind to tumor EV proteins and initiate the generation of Cas12a activators by HCR, assisting in the direct detection of tumor EVs with high sensitivity (Xing et al., 2020).

Deoxyribozymes (DNAzymes) are another type of functional nucleotide that cleaves and releases specific DNA sequences in the presence of certain metal ions. Using this strategy, DNAzymes are integrated with the Cas12a transcleavage system to facilitate the convenient and sensitive detection of ions such as $\mathrm{Pb}^{2+}$ (Li et al., 2020a) and $\mathrm{Na}^{+}$ (Xiong et al., 2020). 


\section{CRISPR-Dx in the COVID-19 pandemic}

The outbreak of the COVID-19 pandemic has caused millions of deaths worldwide and is still disrupting livelihoods and threatening human life and health. Diagnostic tools for the pathogenic SARS-CoV-2 virus that use either immunochromatography or reverse transcription (RT) real-time PCR (RT-qPCR) technology were successfully developed soon after the outbreak (Corman et al., 2020; He et al., 2020). Although the immunology solution is of great convenience, the low accuracy and sensitivity are a concern. PCR, in contrast, is well known for its robustness and accuracy; however, the need for specialized machines and laboratories may prevent it from being used in decentralized scenarios. With the aid of CRISPR technologies, dozens of CRISPRDx systems have been developed, aiming to provide SARSCoV-2 diagnosis with high specificity, sensitivity, rapidness, and convenience (Ding et al., 2020; Fozouni et al., 2021; Ganbaatar and Liu, 2021; He et al., 2020; Huang et al., 2020b; Joung et al., 2020; Ning et al., 2021; Patchsung et al., 2020; Shinoda et al., 2021).

By integrating Cas trans-cleavage reactions with either PCR or isothermal amplification, the CRISPR-Dx methods can further improve the specificity and sensitivity of amplification methods (Huang et al., 2020b; Joung et al., 2020). For example, due to the limitations of RT-qPCR technology, high Ctvalues are frequently encountered during SARS-CoV-2 diagnosis, possibly resulting in both false-positive and false-negative diagnostic results (Li et al., 2020c; Wernike et al., 2021; Xiao et al., 2020). HOLMES was employed to solve this problem, and the RT-qPCR amplicons can be further analyzed by Cas12a-based diagnosis, establishing a specific enhancer for the identification of nucleic acids amplified by a PCR (SENA) diagnostic system with improved sensitivity and specificity (Huang et al., 2020b). Similarly, Cas12a has been combined with RT-RPA to develop a sensitive diagnostic system for SARS-CoV-2 detection (Ning et al., 2021).

LAMP has been demonstrated as an efficient isothermal method for nucleic acid amplification and detection, but it has a high false-positive rate. To solve this problem and achieve convenient SARS-CoV-2 detection, researchers developed the one-pot STOPCovid.v2 method, which resembles HOLMESv2 in principle (Li et al., 2019a), with the use of LAMP for preamplification and thermophilic Alicyclobacillus acidophilus Cas12b (AapCas12b) for transcleavage and signal output (Joung et al., 2020). Because AapCas $12 b$ is resistant to high temperature, STOPCovid.v2 can be carried out at $60^{\circ} \mathrm{C}$ to facilitate LAMP preamplification and exhibits a higher detection sensitivity than HOLMESv2, which is performed at $55^{\circ} \mathrm{C}$ (Li et al., 2019a). In addition, magnetic beads are used to enrich the total nucleic acids from the lysed samples, which further enhances the detection sensitivity of STOPCovid.v2 (i.e., 33 copies per milliliter) far beyond the requirement of the Centers for Disease Control and Prevention (CDC) for the RT-qPCR test (Joung et al., 2020). As no more expensive instrument than a heat block is required, STOPCovid v2 can be used to facilitate COVID-19 diagnosis in areas lacking sophisticated medical instruments.

Several amplification-free CRISPR-Dx systems using Cas trans-cleavage activities have been successfully developed and shown competitiveness in COVID-19 diagnosis (Fozouni et al., 2021; Liu et al., 2021a; Shi et al., 2021; Shinoda et al., 2021; Tian et al., 2021; Yue et al., 2021). To increase the detection sensitivity, more than one crRNA targeting the SARS-CoV-2 RNA sequence can be used, and amplificationfree detection can be accomplished within 30 min with a sensitivity of approximately 100 copies per microliter (Fozouni et al., 2021); however, this sensitivity may still require improvement to meet the CDC criteria. Alternatively, unamplified target nucleic acids can be remarkably concentrated in picolitre-sized droplets with the ultralocalization of Cas13 trans-cleavage reactions by means of droplet microfluidics (Tian et al., 2021), and the system shows extremely high sensitivity and enables the absolute digital quantification of target RNAs at the single-molecule level without preamplification.

\section{Summary}

CRISPR-Dx began in 2015 when the CASFISH system with CRISPR-dCas9 was developed (Deng et al., 2015). The discovery of the trans-cleavage activities of Cas12 and Cas13 has aroused further interest in developing next-generation molecular diagnostic tools (Chertow, 2018). Because of the outstanding performance, two CRISPR-Dx products were issued an Emergency Use Authorization (EUA) for COVID-19 diagnosis last year, including the Cas13-based Sherlock CRISPR SARS-CoV-2 Kit by the Food and Drug Administration (FDA) in the US and the Cas12-based 2019nCoV Nucleic Acid Detection Kit (CRISPR Immunology) by the National Medical Products Administration (NMPA) in China. With an increasing number of in vitro diagnostic (IVD) products on the market, CRISPR-Dx might eventually enter clinical use.

Because of the specificity and the trans-cleavage activity of Cas proteins, CRISPR-Dx systems have shown great advantages in detection, including sensitivity, accuracy, rapidness, and portability (Li et al., 2019b). Several amplificationfree CRISPR-Dx systems have been developed using either Cas12 or Cas13 (Fozouni et al., 2021; Liu et al., 2021a; Shinoda et al., 2021; Tian et al., 2021) with optimization of the reaction system and readout-related sensitivity. They show great potential in the diagnosis of pathogens and tumors. Although Cas9 can be integrated with a graphenebased field-effect transistor to achieve the amplification-free 
detection of target nucleic acids (Balderston et al., 2021; Hajian et al., 2019), it might be less competitive in cost. In addition, the isothermal trans-cleavage activity has brought both sensitivity and rapidness to CRISPR-Dx methods. This may cause difficulties in the accurate quantification of target nucleic acids. In accordance with the principles of digital PCR, CRISPR-Dx is combined with droplet- or chip-based partition technology to achieve absolute quantification (Shinoda et al., 2021; Tian et al., 2021; Yue et al., 2021).

Multiplexing is another important factor that influences the application of MDx technology. Although SHERLOCKv2 supports the one-pot detection of four targets with the use of Cas 13 orthologs and Cas 12 proteins (Gootenberg et al., 2018), there are still challenges in multiplexed detection, including the fragility of the system using multiple enzymes and the relatively high background signals. Therefore, the majority of CRISPR-Dx trans systems detect one target in one reaction system, and novel solutions are needed to facilitate robust multiplexed diagnosis.

It is expected that novel CRISPR-Dx methods will be developed alongside the characterization of an increasing number of CRISPR/Cas systems. For example, a recent study found that crRNAs can be produced from cellular RNAs after being hybridized to trans-activating crRNA (tracrRNA) and cleaved by Cas9 in Campylobacter jejuni. Based on this finding, a reprogrammed tracrRNA (Rptr) was designed to cleave a desired RNA target to generate a functional crRNA, which together with Rptr guided Cas9 to cut target DNA. The method leveraging engineered tracr RNAs and on-target DNAs for parallel RNA detection (LEOPARD) was developed to enable the one-pot detection of multiplexed RNA sequences with single-base resolution (Jiao et al., 2021). However, to facilitate the use of LEOPARD for molecular diagnosis, the gel-based readouts in LEOPARD could be replaced by more convenient approaches such as LFA or fluorescence in the future.

\section{Therapeutic gene editing}

The goal of gene therapy is to use various strategies to correct or manipulate gene expression to restore defective cell functions or reinforce the biological properties of normal cells to ameliorate symptoms or cure diseases with minimal adverse events. Since the first clinical study undertaken at the US National Institutes of Health in 1990, more than three decades of exploration have built a solid basis for gene therapy. The total number of clinical studies in this area has surpassed 2,500, targeting monogenic diseases, complex neurodegenerative disorders, infectious diseases, cancer, etc. Since 2015 , more than 10 gene therapy products have been approved worldwide for cancer and genetic disorders. Like the commentary published in Science, we would like to say "gene therapy comes of age" (Dunbar et al., 2018).

There are two basic delivery strategies to fulfill the goal of gene therapy: (i) introducing an integrating vector with genetic material into proliferating cells (usually hematopoietic cells) through an ex vivo process so that the donated DNA will be replicated during cell division and therefore passed to every daughter cell; or (ii) introducing exogenous nucleic acids into postmitotic cells via nonintegrating vectors through in vivo methods to achieve sustained expression of the target gene in the entire lifespan of the cells. However, the genetic modification of patient cells is potentially dangerous, so safety issues are the most critical factors that have raised serious concerns in gene therapy clinical trials. The data accumulated since the 1990s make it clear that the risks fall into two main aspects. The risk of insertional mutagenesis is a recognized disadvantage of integrating vectors. The clinical ramifications of insertional mutagenesis were confirmed by the development of $\mathrm{T}$ cell leukemia in four young patients who received retrovirus-mediated gene therapy for $\mathrm{X}$-linked severe combined immunodeficiency (SCID) (Hacein-Bey-Abina et al., 2008). Second, immune-mediated rejection is related to in vivo vector vehicles (Shirley et al., 2020). For example, adenoviral vectors (AdVs) may induce a strong immune response that leads to life-threatening multiple organ system failure (Raper et al., 2003). Currently, vectors that cause severe immune side effects have been weeded out. Lentiviral vectors have become the leading system for ex vivo gene transfer (Naldini, 2011), while in vivo therapies usually use AAV vectors (Wang et al., 2019a; Witzigmann et al., 2020). AAV is considered a relatively safe vector since it induces minimal immune responses. Its vector DNA predominantly forms a stable episome that prevents insertional mutagenesis at the cost of expression duration. The limited duration of AAV vector and random insertioninduced lentiviral vector tumorigenic potential are major deficiencies of "traditional" gene therapy.

In contrast to traditional gene therapy technologies, which can mediate only "gene addition" through various delivery vectors, genome editing technologies, such as ZFNs, meganucleases, TALENs and Cas9 nucleases, are able to generate permanent, precise and flexible gene ablation, insertion or correction at one or multiple target genes. The versatility and simplicity of the revolutionary programmable nucleases, especially the CRISPR/Cas9 system, quickly made them the most critical technology in gene therapy. This chapter provides a brief summary of recent progress in genome editingmediated gene therapy, from bench to bedside.

\section{In vivo gene therapy}

Genetic diseases of the nervous system, vision, and hearing loss as examples

Combining the progress of several research areas, including 
molecular biology, human genetics, delivery approaches, and clinical translation, retinal gene therapy is progressively being recognized as a potential intervention with great potential for treating inherited retinal dystrophies (IRDs). Early proof-of-concept clinical trials (NCT00481546, NCT00516477, and NCT00643747) used AAV vectors to deliver the gene encoding a $65-\mathrm{kD}$ retinal pigment epithelium-associated protein (RPE65) into the eyes of patients carrying biallelic RPE65 recessive mutations (Bainbridge et al., 2008; Cideciyan et al., 2009; Maguire et al., 2008). Subsequent studies and clinical trials (NCT00481546, NCT00643747, NCT01208389, and NCT00999609) demonstrated a statistically significant and clinically meaningful difference between the intervention and control groups without significant safety issues (Bainbridge et al., 2015; Bennett et al., 2012; Bennett et al., 2016; Jacobson et al., 2015; Maguire et al., 2009; Russell et al., 2017). All these efforts allowed the FDA and EMA to approve Luxturna (Spark Therapeutics) at the end of 2017 as a gene therapy product to treat the monogenic disorder Leber congenital amaurosis (LCA-2). The approval of Luxturna fueled additional trials for other IRDs (Chiu et al., 2021) focused on LCA (NCT00481546, NCT02946879), retinitis pigmentosa (RP) (NCT03328130, NCT04611503), X-linked retinitis pigmentosa (XLRP) (NCT04671433, NCT03316560), and achromatopsia (NCT02341807, NCT02077361). In addition to AAV-mediated gene therapy, a few studies have tried to correct the mutation at the mRNA level via antisense oligonucleotides to restore the splicing of mutant pre-mRNA (NCT03913143) (Chiu et al., 2021).

Most recently, preclinical studies have demonstrated the promising application of CRISPR-Cas9-based therapeutics in LCA, RP, or other retinal diseases. After Zhong et al. (2015) used the CRISPR-Cas9 system to validate that the expression of $\mathrm{KCNJ} 13$ is related to photoreceptor survival and plays a role in LCA pathogenesis, researchers employed it to edit a disease-associated gene in LCA. IVS26, an adenine-to-guanine point mutation (c. $2991+1655 \mathrm{~A}>\mathrm{G})$ in intron 26 , is a common mutation involved in LCA subtype 10 (LCA10) (Boye et al., 2014; den Hollander et al., 2006). This mutation forms a new splicing donor site in the mRNA, creating a premature stop codon that completely inactivates CEP290. Thus, the Cas9 proteins from $S$. pyogenes or $S$. aureus with dual sgRNAs were tested to remove or reverse the mutation in the CEP290 gene to correct splicing and then generate a functional CEP290 transcript. The experimental data suggested that Cas9-based removal of the pathogenic variant in the intron of CEP290 could be a potential treatment strategy for LCA (Maeder et al., 2019; Ruan et al., 2017). In the first Cas9-mediated in vivo gene therapy trial, Allergan and Editas Medicine initiated the first patient dosing in the phase $1 / 2$ clinical trial of AGN-151587 (EDIT101) for the treatment of LCA10 (NCT03872479) with the above strategy. In this trial, SaCas9 and sgRNAs were packaged in AAV vectors and delivered via subretinal injection.

As a monogenic hereditary retinal disorder, RP causes irreversible blindness due to pathogenic (loss-of-function or gain-of-function) mutations in more than 50 candidate genes. Therefore, the CRISPR-Cas9 genome editing system is a prominent tool to treat autosomal dominant RP (adRP). For example, in rodent models, a recent study employed CRISPR-Cas9 to knock out the mutant allele of the rhodopsin $(R H O)$ gene, which is vital for retinal cell survival, in rodent models (Bakondi et al., 2016). The disruption of the murine S334ter mutation (Rho(S334)) halted retinal degeneration and improved visual function. In three other similar human studies, the modification $\mathrm{P} 23 \mathrm{H}$ in the $R H O$ gene by CRISPR-Cas9 significantly reduced the abundance of mutant RHO protein (Giannelli et al., 2018; Latella et al., 2016; Li et al., 2018a). Moreover, this genome editing system has been utilized to understand the molecular biology of RP for clinical interventions. For example, a nonsense point mutation (Y347X) and an Xmv-28 insertion in the phosphodiesterase $6 B(P D E 6 B)$ gene are associated with RP in a rodless (rd1) mouse model. One study demonstrated that the outer nuclear layer (ONL) could be restored after Cas9 repair of the Y347X mutation (Wu et al., 2016). In another study, Cas9 was assembled with the E. coli RecA protein to repair this mutation in rd1 mice. The most important contribution of this study is the demonstration of the feasibility of Cas9induced HDR to treat $\mathrm{rd} 1$ disease and of the Cas9/RecA system to improve HDR efficiency in vivo, which may also benefit the treatment of other diseases (Cai et al., 2019).

A large number of people suffering from hearing loss would also benefit from gene-editing technologies, since half of hearing loss is caused by genetic mutations, among which $20 \%$ are gain-of-function mutation (Müller and Barr-Gillespie, 2015). Theoretically, these mutant alleles can be targeted by CRISPR-Cas9 to correct the production of malfunctional proteins. TMC1 is an essential protein for the conversion of mechanical signals to bioelectrical signals (Pan et al., 2013). The TMC1 c.T1253A mutation can cause the hearing loss disorder DFNA36, a dominant genetic disorder. To test whether disruption of the c.T1253A allele via Cas9 is able to rescue hearing loss in a DFNA36 mouse model. Gao et al. (2018) utilized the lipid delivery system to transmit Cas $9 / \mathrm{sgRNA}$ into the inner ear of a mouse model. They designed a series of sgRNAs containing the TMC1 mutation at the PAM proximal region to prevent cleavage of the WT TMC1 allele. Their results showed that the treatment robustly reduced progressive hearing loss, with higher hair cell survival rates and lower auditory brainstem response thresholds in the neonatal mouse model. In contrast to Gao's strategy, György et al. (2019) used AAV2/Anc80 as the delivery vehicle and SaCas9-KKH as the targeting nuclease. 
They placed the TMC1 mutation in the PAM region to selectively target the mutant $\mathrm{TMC} 1$ allele and found that the postnatal delivery of AAV-SaCas9-KKH/sgRNA durably preserved the hearing capability of the mice for at least one year. However, some TMC1 mutations, for example, c. A545G, result in a recessive loss-of-function point mutation, which is not suitable for the above disruption strategy. To correct the point mutation and rescue the hearing ability of TMC1 c.A545G mutated mice, Yeh et al. (2020) tested several base editors. They chose the most efficient AIDBE4max as the effector and selected the split-intein delivery system to fit the base editor into a dual AAV package system. They achieved approximately $2.3 \%$ desired base correction in Tmc1 with ear injection of the dual AAV. Importantly, mice with this correction rate could produce normal Tmc1 mRNA that reached $51 \%$ of the WT level, which restored the low-frequency hearing of the mice 4 weeks after the treatment.

Both retinal and hair cells are suitable targets for genome editing-based gene therapy, since they can be efficiently transduced through local injection via AAV, and the dose of AAV required is much lower than the systemic injection doses required for other diseases. Notably, even limited editing efficiency demonstrated impressive efficacy in these studies. This suggests that editing efficiency may not be highly demanded in these applications.

\section{Gene editing in liver gene therapy}

The liver is one of the most targeted organs for gene therapy because many metabolic diseases are caused by the dysfunction of genes related to the liver. In addition, the liver can be efficiently transduced by viral or nonviral vectors. In early clinical trials, researchers utilized the gene addition technique to deliver healthy genes into the liver via lentivirus or AAV. Several clinical trials aiming to cure hemophilia had already shown encouraging results before the new geneediting tools were developed (Nguyen et al., 2021). The development of efficient delivery technologies and the advances in gene editing tools have resulted in practical approaches to treat several genetic diseases via NHEJ or, in some cases, even via HDR in the liver.

The PCSK9 gene, which is expressed exclusively in the liver, is one of the candidate genes for treating cardiovascular diseases (Seidah et al., 2014). Evidence has shown that a gain-of-function variant of PCSK9 is linked to a high level of blood low-density lipoprotein cholesterol (LDL-C) (Seidah et al., 2014), a primary cause of cardiovascular diseases. In contrast, people carrying loss-of-function PCSK9 variants are asymptomatic, showing lower levels of LDL-C and reduced risks of cardiovascular diseases (Guo et al., 2020b). Ding et al. (2014a) delivered Cas9/SgRNA targeting Pcsk9 into the mouse liver via injection of AAV8 particles. They observed a mutagenesis rate of over $50 \%$ in $P \operatorname{csk} 9$, resulting in ten times lower plasma Pcsk 9 and a $35 \%-40 \%$ decrease in blood LDL-C. Soon after Ding's promising results, this strategy's long-term efficacy and safety issues were further studied in mice and nonhuman primates via different programmable nucleases, including ZFN (Conway et al., 2019) or meganucleases (Wang et al., 2018b). The results were encouraging, as PCSK9 was efficiently and durably knocked out in those experiments. All studies demonstrated a considerable reduction in blood LDL-C, and no significant offtarget events or adverse effects were observed.

Apo (apolipoprotein) C3 is another risk factor associated with a number of cardiovascular diseases through mediating the metabolism of triglyceride (TG)-rich lipoproteins. Lossof-function mutations are associated with low levels of TG and a decreased incidence of cardiovascular diseases (Crosby et al., 2014). ApoC3 knockout in rabbits using ZFN has shown that ApoC3 deficiency significantly accelerates the catabolism of TG-rich lipoproteins in the liver and leads to the resistance of $\mathrm{KO}$ rabbits to cholesterol diet-induced hyperlipidemia and atherosclerosis (Yan et al., 2020; Yang et al., 2013a). A recent work using CRISPR/Cas9 to inactivate ApoC3 also showed a protective effect against atherosclerosis in hamsters (Guo et al., 2020a). These results support therapeutic inactivation of $\mathrm{ApoC} 3$ as a strategy for the treatment of hyperlipidemia and atherosclerosis.

For other metabolic liver diseases, such as hereditary tyrosinemia-I (HT-1) and primary hyperoxaluria type I (PH-I), targeting an upstream gene to reprogram the metabolic pathway could also be an effective approach. In HT-I, mutations in the gene fumarylacetoacetase (FAH) result in the accumulation of tyrosine and other toxic metabolic intermediates, such as succinylacetone (Shao et al., 2014). The current treatment for HT-I requires the daily ingestion of nitisinone, an inhibitor of 4-hydroxyphenylpyruvate dioxygenase (HPD), which is the upstream enzyme for tyrosine degradation (Holme and Lindstedt, 1998). The inhibition of HPD will convert the accumulation of toxic succinylacetone into its upstream intermediate - 4-hydroxyphenylpyruvate (4HPP), which is observed in the more benign disease HT-III (Pankowicz et al., 2016). Following the above mechanism, Pankowicz et al. (2016) delivered Cas9/sgRNA targeting the Hpdl gene into the murine liver via the hydrodynamic tail vein injection of plasmids. A week after the injection, up to $20 \%$ of the Hpd allele was deleted. As the edited hepatocytes have a growth advantage over diseased cells, they quickly repopulated $99 \%$ of hepatocytes 8 weeks after treatment.

Similar to the treatment of HT-I, metabolic reprogramming was performed in the treatment of PH-I. In PH-I, a mutation in the AGXT gene leads to the failure of glyoxylate to glycine conversion. The accumulated glycosylate is oxidized by lactate dehydrogenase (LDH), causing the overproduction of highly insoluble calcium oxalate $(\mathrm{CaOx})$ crystals in the kidneys (Zheng et al., 2020). An ongoing clinical trial to treat 
PH-I targeting the HAO1 gene via RNA interference reduces the expression of glycolate oxidase, an upstream enzyme that generates glyoxylate (Garrelfs et al., 2021) (NCT03681184). Soon, the therapeutic effect of HAO1 disruption via CRISPR/Cas9 was also validated in mice (Zabaleta et al., 2018) and rats (Zheng et al., 2020). In both studies, the treated animals exhibited alleviated symptoms, showing that this strategy has great promise. Notably, although the use of CRISPR/Cas9 or other nucleases to treat genetic diseases via deletion of endogenous genes to reprogram metabolic pathways is effective, the long-term safety issue of this strategy has yet to be extensively examined. Due to these concerns, current clinical trials have focused mainly on RNAi strategies to treat those diseases through in vivo gene manipulation. However, an advantage of programmable nucleases is that they avoid the repeat delivery of therapeutic vectors, which could be painful and costly to patients. Recently, Intellia and Regeneron revealed milestone clinical data on therapy for transthyretin (ATTR) amyloidosis, a disease caused by misfolding and deposition of the transthyretin protein. Their study delivered nanoparticles encapsulating the mRNA of Cas9 and sgRNA targeting the transthyretin (TTR) gene into the patients. Taking advantage of mRNA avoids the long-term in vivo expression of Cas9, thus minimizing the possibilities of off-target events and exogenous DNA integration. More importantly, they demonstrated that a single dose of NTLA-200 was able to reduce transthyretin by $87 \%$ on average, which lasted for over a year without relapse (NCT04601051) (Gillmore et al., 2021). As the capacity of AAVs is limited (approximately $4.7 \mathrm{~kb}$ ), it is difficult to deliver genome-editing materials through a single AAV vector. The clinical success of LNP-based Cas9/ sgRNA delivery is a critical landmark for both mRNA medicine and genome editing therapeutics, as the short life of mRNA would greatly reduce the potential off-targeting effects, and the LNPs would not induce immune tolerance, which is the problem with repetitive dosing of AAV products in patients with AAV antibodies.

In vivo gene therapy via DNA fragment integration may be the greatest challenge in this field because conventional DNA integration use the HDR pathway, which has an extremely low intrinsic frequency, especially in quiescent somatic cells. However, in some diseases, partial correction of the mutated genes might significantly and durably relieve their symptoms. A case in point is the potential gene therapy for hemophilia. Guan et al. (2016) achieved a 0.5\% correction of the $f 9$ gene through hydrodynamic tail vein injection of the Cas9/sgRNA plasmid and a donor fragment into the mouse liver. They found that the treated mice had shortened blood coagulation time and survived the tail-clip challenge, although only a tiny portion of the liver cells was corrected. Similar results were observed in the study for hemophilia A (Chen et al., 2019a). To further increase the integration fre- quency of large DNA fragments, Zhang et al. (2019a) harnessed NHEJ-mediated knockin and an AAV donor fragment to treat hemophilia A. They achieved an in vivo knockin frequency of $2 \%$ for the $B D D F 8$ donor fragment (over $4.4 \mathrm{~kb}$ ). Since AAV vectors are commonly used as delivery vectors for nucleases and donors in gene therapies, an increase in delivery efficiency might elevate both cleavage and HDR efficiency. Yin et al. (2020) attempted to codeliver an AAV receptor (AAVR) in a study on gene therapy for phenylketonuria (PKU). The coexpression of AAVR significantly increased the in vivo HDR efficiency by up to 20fold and ameliorated the blood Phe level, although the longterm effects of AAVR overexpression need full investigation in the future.

It is worth mentioning that most of the above editing strategies are based on the creation of DSBs in the genome. However, due to the potential risks of DSBs, researchers are making gene-editing tools more precise on the one hand, while on the other hand, they are looking for potential substitutes for the programmable nucleases. The invention of base editors offered scientists alternative choices. Unlike conventional programmable nucleases, base editors produce few DSBs in the genome, and their efficiency outperforms the traditional HDR pathway. Villiger et al. (2018) attempted to treat a PKU mouse model via in vivo base editing. They engineered the cytidine base editor (ABE) into an intein-split system to fit each portion of the base editor into the capacity limitation of the AAV particles. The AAV was then delivered via tail vein injection. During the next 26 weeks, they observed a continuous increase in the DNA and mRNA correction rates and a significant drop in the L-Phe level in the treated mice, indicating the feasibility of this strategy. Moreover, the high efficiency and flexibility of base editors give researchers a greater capability to cure diseases. Beyond correcting mutated base pairs, scientists are able to manipulate mRNA splicing, including creating de novo start codons and installing stop codons in the genome. In 2020, the first gene therapy study evaluating $\mathrm{ABE}$ was performed by Song et al. They combined ABE and the hydrodynamic tail vein injection technique to restore a mutated splice donor site in the $f a h$ gene in an HT-I mouse model (Song et al., 2020). In the same year, Yang et al. (2020) managed to create a de novo "ATG" via in vivo base editing, which helped to read through the mutant fah gene in an HT-I mouse model. Recently, Rothgangl et al. (2021) used an ABE to target the splice donor site in intron 1 of the $P C S K 9$ gene in macaques. Their strategy led to a decrease of $95 \%$ in the PCSK9 level within $48 \mathrm{~h}$, and more importantly, a corresponding decrease of $58 \%$ in LDL was observed as well.

While base editors are efficient and effective in gene therapy studies, two major concerns affect the research field. First, the bystander editing effect could narrow the potential target scope of the base editors. Second, with the emergence 
of more sensitive detection methods, new off-target phenomena of base editors could be discovered (Lei et al., 2021). Therefore, a systematic off-target event inspection is suggested for each target site before a clinical trial is initiated.

\section{Ex vivo gene therapy}

\section{Gene therapy in hematopoietic stem cells}

Due to the maturation of blood separation techniques, ex vivo gene therapies mainly adopt hematopoietic progenitor/stem cells (HSPCs) as the target. Initially, clinical trials took advantage of gene addition technology to treat inherited immunodeficiency disorders, including X-linked SCID and adenosine deaminase (ADA) deficiency. In the above trials, healthy donor genes encoding the $\gamma \mathrm{c}$ or ADA protein were delivered into the immune cells or later into the HSPCs of the patients via retroviral or lentiviral particles. Treated cells were then transplanted back to the patients to supplement the proteins needed as the building blocks of the immune system. The overall efficacy of the first attempts was quite exciting. Although the therapeutic effect lasted only months in some cases, others demonstrated that the concept of longterm gene augmentation was feasible (Aiuti et al., 2002; Aiuti et al., 2009).

Based on prior knowledge, when it became plausible that genetic disorders could be cured by HSPC manipulation and transplantation, $\beta$-thalassemia and sickle cell disease were the first diseases to be targeted. Both diseases are caused by mutations in the gene encoding $\beta$-globin, which normally forms the hemoglobin tetramer with $\alpha$-globin. Patients with a severe type of both diseases have low oxygen-carrying hemoglobin and depend on regular blood transfusions (Thompson et al., 2018). Reactivation of the silenced gene $\gamma$ globin, which acts as a substitute for $\beta$-globin, could cure these two diseases. More importantly, studies suggested that $\gamma$-globin could be reactivated by creating indels in the regulatory elements of the $\gamma$-globin promoter $(-112$ to -117$)$ region (Traxler et al., 2016) or of the +58 enhancer of a $\gamma-$ globin repressor, namely, BCL11A (Canver et al., 2015; Wu et al., 2019). Immediately, various gene-editing studies were performed using HSPCs to prove this concept via ZFN (Bjurström et al., 2016), TALEN (Humbert and Kiem, 2015), or, in most cases, via CRISPR/Cas9 (Canver et al., 2015; Wu et al., 2019). In addition, edited HSPCs from patients were viable and functional in nonhuman primates (Humbert and Kiem, 2015; Humbert et al., 2018). In 2018, gene editing companies, including Sangamo and CRISPR Therapeutics, initiated phase $1 / 2$ clinical trials evaluating the efficacy and safety of editing the BCL11A enhancer to treat sickle cell disease (NCT03745287) or $\beta$-thalassemia (NCT03432364, NCT03655678). A year later, similar trials targeting BCL11A were launched in China (NCT04211480, NCT04390971). The results from these trials are astounding.
All patients receiving the treatment are able to produce sufficient fetal hemoglobin, which helps them become independent of transfusions (Frangoul et al., 2021). In addition to the $\gamma$-globin reactivation strategy for $\beta$-thalassemia, scientists discovered other methods to restore $\beta$-globin. For example, in $\beta$-thalassemia IVS-654, a C654-A transition leads to aberrant $\beta$-globin pre-mRNA splicing, preventing the synthesis of $\beta$-globin protein. Using programmable nucleases, scientists successfully restored $\beta$-globin mRNA splicing by targeting intron 2 of the $\beta$-globin gene (Xu et al., $2015)$. Another more direct strategy for $\beta$-globinopathies is to correct the disease-causing mutations via CRISPR/Cas9stimulated HDR (Dever et al., 2016; Wilkinson et al., 2021). However, since HDR has a low spontaneous frequency, whether enough corrected HSPCs can be enriched is the key to the success of the treatment. Currently, a clinical trial based on the HDR strategy has been initiated (NCT04819841) by Graphite Bio. Notably, in addition to nuclease-based gene-editing tools, base editors are beginning to play an important role in the treatment of $\beta$-globinopathies. Reports have revealed that it is possible to reactivate $\gamma$-globin by editing the BCL11A +58 enhancer (Zeng et al., 2020 ) or HBG1/2 promoter region (Wang et al., 2020b). Recently, results obtained by Gregory et al. demonstrated that through an adenine base editor (ABE8e-NRCH), the GAG(E) to GTG(V) mutation, which causes sickle cell disease, could be efficiently converted to a benign Makassar variant (Newby et al., 2021). Since ABEs are able to convert base pairs efficiently without inducing DSBs, which is superior to Cas9-mediated mutagenesis, the sgRNA-independent off-targeting effects of the base editors still require close attention. Scientists are devoting attention to improving base editing technology through engineering base editors and developing delivery technologies for Cas9 protein or mRNA to reduce off-target edits.

\section{Gene editing in primary T cells for immunotherapy}

In 1994, zinc finger proteins were first shown to repress the function of oncogenic BCR-ABL fusion sequences by directly binding DNA (Choo et al., 1994). Later, zinc finger proteins were coupled with nuclease to mediate gene disruption in various cell lines and primary cells, such as T cells (Urnov et al., 2005). A double genetic disruption of TCR- $\beta$ and $\alpha$-chain genes followed by the lentiviral transfer of a new TCR into human primary T cells was first reported in 2012 (Provasi et al., 2012). The edited T cells treated with ZNFs lacked surface expression of CD3-TCR and expanded with the addition of interleukin-7 (IL-7) and IL-15. Another study also disrupted TRAC, TRBC1, and TRBC2 using ZFNs to generate universal chimeric antigen receptor (CAR) T cells for multiple allogenic patients (Torikai et al., 2012). Later, the same group used ZFNs to knock out the HLA-A gene in CAR-T cells, further improving the universal CAR-T 
strategy (Torikai et al., 2013). Another study knocked down the glucocorticoid receptor using ZFNs in modified cytolytic $\mathrm{T}$ lymphocytes (CTLs), which were generated by introducing a chimeric T-cell receptor consisting of an extracellular IL13 domain and a cytoplasmic CD3 domain (IL13-zetakine). Thus, ZFN-modified glucocorticoid-resistant IL13-zetakinetargeted CTLs retained their function in cancer patients regardless of glucocorticoid treatment (Reik et al., 2008).

Only one year after the elucidation of TALE-DNA recognition codes, TALEs were designed and linked to FokI to establish the TALEN platform. It is worth mentioning that after scaffold optimization, TALE showed comparable ontarget activity to benchmark ZFNs but lower cytotoxicity, at least at three human loci tested (CCR5, IL2RG, AAVS1) (Mussolino et al., 2011; Mussolino et al., 2014). The first TALEN-mediated cell therapy to enter clinical trials has focused mainly on the generation of universal CAR-T cells. Cellectis SA has developed an allogeneic approach named universal chimeric antigen receptor (CAR) T-cells targeting CD19 (UCART19), which aims to offer standardized therapeutics with good consistency and immediate availability. Genetic manipulation involves TALEN mRNA-mediated gene disruption against TCR $\alpha$ constant gene (TRAC) and CD52. The preliminary results reported two phase 1 clinical trials to evaluate the safety and antileukemic activity of UCART19 in children and adults with relapsed or refractory B-cell acute lymphoblastic leukemia (Benjamin et al., 2020). Fourteen $(67 \%)$ of 21 patients had a complete response or complete response with incomplete hematological recovery $28 \mathrm{~d}$ after infusion. Patients not receiving CD52 targeting alemtuzumab $(n=4)$ showed no UCART19 expansion or antileukemic activity. The median duration of response was 4.1 months, with ten $(71 \%)$ of 14 responders proceeding to a subsequent allogeneic stem-cell transplant. Progression-free survival at 6 months was $27 \%$, and overall survival was $55 \%$ (NCT02808442, NCT02746952). A similar strategy was applied to other CAR-T products in the Cellectis pipeline, such as UCART123 targeting CD123, the primary low-affinity subunit of the IL-3 receptor, which is highly expressed in some hematological cancers. The phase I UCART19 trial was underway for AML (NCT03190278). Several other TALEN-edited allogeneic UCART products have also entered phase I clinical trials, including UCART22, UCARTCS1, and ALLO-751 (NCT040993596, NCT04142619, NCT04150497).

The first CRISPR/Cas9-mediated multiplex gene editing in CAR-T cells was reported by the June group and Wang group (Liu et al., 2017b; Ren et al., 2017). TRAC, B2M, and PDCD1 were chosen as target genes in both studies. Electrotransfer of Cas9 mRNA-sgRNAs was applied in June's study. After optimizing the electroporation conditions for delivery, $80 \%$ single-gene KO efficiency and $65 \%$ dKO efficiency were obtained. Instead of Cas9 mRNA, the Wang group delivered a RNP consisting of the Cas9 protein in complex with sgRNAs and achieved efficient single- and multiple-gene KO. In both studies, potent antitumor efficacy of gene-edited CAR-T cells was demonstrated in both in vitro assays and tumor xenograft mouse models. Later, CRISPR/ Cas9-mediated $\mathrm{KO}$ of various genes in CAR-T cells or $\mathrm{CD} 8^{+}$ $\mathrm{T}$ cells to eliminate various immune checkpoint genes (PDCD1, LAG3, CTLA4, DGK $\alpha$ ) (Jung et al., 2018; Rupp et al., 2017; Zhang et al., 2019b; Zhang et al., 2017b), to inhibit immune-suppressive signaling (TGF $\beta$, A2AR) (Li et al., 2020b), or to generate allogeneic CAR-T cells for the treatment of $\mathrm{T}$ cell hematologic malignancies (CD7\&TRAC) was reported (Cooper et al., 2018). It is worth noting that triphosphate at the $5^{\prime}$ end of IVT (in vitro transcription)-sgRNA could initiate the innate immune responses of primary $\mathrm{T}$ cells, impairing cell viability. Eliminating the triphosphate at the $5^{\prime}$ end of IVT-sgRNA by CIP or using chemically synthesized sgRNA solved this problem (Kim et al., 2018; Mu et al., 2019). As with TALENs, the first CRISPR trial for geneedited cells was also T cells. They were first applied to treat a patient with advanced non-small cell lung cancer (NCT02793865). Many subsequent registered CRISPR trials focused on the use of autologous T-cells, knocking out the immune checkpoint inhibitor programmed cell death-1 (PD1) prior to reinfusion. Seven clinical trials targeting PD1 are currently in phase 1 (NCT02793856, NCT02863913, NCT02867332, NCT02867345, NCT03545815, NCT03747965, NCT04417764), mainly in China.

\section{Gene editing in infectious diseases}

The main strategy of antiviral therapeutics involves altering the host genes required for viral infection or targeting the viral genes essential for viral productivity (Kennedy and Cullen, 2017). The strategy of genome editing-based HIV therapy is to edit the gene encoding the receptor of HIV infection in $\mathrm{CD}^{+} \mathrm{T}$ or $\mathrm{CD} 34^{+}$hematopoietic stem/progenitor cells and reinfuse the modified cells into patients.

The CCR5 gene encodes the HIV coreceptor, which is utilized by HIV-dominant strains. Homozygosity of the naturally occurring 32 bp deletion (delta32) in CCR5 confers resistance to HIV-1 infection (Liu et al., 1996), and people heterozygous for delta32 have a slower progression after HIV infection, suggesting the feasibility of obtaining HIV resistance by mutating CCR5 using genome editing technologies (Huang et al., 1996; Zimmerman et al., 1997). The pioneering work in this field was done using ZFNs. In a preclinical study, Perez et al. delivered ZFN into human primary $\mathrm{CD}^{+} \mathrm{T}$ cells using a chimeric Ad5/F35 adenoviral vector and achieved a 50\% CCR5 disruption rate. HIV-1infected mice engrafted with CCR5-edited T cells had lower viral loads and higher $\mathrm{CD}^{+}$T-cell counts than mice engrafted with control T cells (Perez et al., 2008). Based on this promising therapeutic outcome, the first clinical usage of 
gene-edited cell therapy was conducted in 2009 by the University of Pennsylvania in collaboration with Sangamo Therapeutics, aiming to disrupt CCR5 by using ZFNs in autologous $\mathrm{CD}^{+}{ }^{+}$T-cells of HIV patients (NCT00842634) (Tebas et al., 2014). The clinical results were revealed in 2014 with only 1 serious adverse event due to a transfusion reaction, proving that the method was safe. After treatment, blood HIV DNA was decreased in all patients, and HIV RNA became undetectable in one patient, suggesting the feasibility of clinical application of gene editing. In addition to $\mathrm{CD} 4^{+} \mathrm{T}$ cells, CD $34^{+}$HSPCs were also engineered to provide a more durable source of HIV-resistant T cells (Allen et al., 2018). Clinical trials using ZNFs (NCT02500849) and CRISPR/ Cas9 (NCT03164135) to disrupt CCR5 in CD34 ${ }^{+}$HSPCs have also been reported (Xu et al., 2019). Although the successful transplantation and long-term engraftment of CRISPR-edited HSPCs were observed, the percentage of CCR5 disruption in lymphocytes was only approximately $5 \%$, which indicates the need for further improvement of this approach (NCT03164135). In addition, TALEN was used to disrupt CCR5, with relatively lower cytotoxicity and higher specificity than ZFNs (Mussolino et al., 2014).

In addition to CCR5, C-X-C chemokine receptor 4 (CXCR4) is an important coreceptor for CXCR4-tropic HIV-1. Thus, HIV-resistant $\mathrm{CD}^{+} \mathrm{T}$ cells that simultaneously inactivate the coreceptors CCR5 and CXCR4 confer protection against HIV that uses either of the coreceptors (Didigu et al., 2014). Similar ex vivo work was also performed using the CRISPR/Cas9 platform (Hendel et al., 2015; Mandal et al., 2014). Instead of direct disruption of the viral genome, researchers have also created alternative methods to eliminate HIV infection. For example, taking advantage of CRISPRa, a gene activation system that consists of a catalytically dead Cas9 and a transcriptional activation domain, scientists are able to reactivate HIV from its latency state (Ji et al., 2016; Saayman et al., 2016). As a result of latency reversal, cells that carry dormant HIV are exposed to immune cells and eliminated.

Infection with high-risk human papillomavirus (HR-HPV) is the main cause of cervical cancer, usually due to the persistent expression of HR-HPV oncogenes E6 and E7. Using gene editing to disrupt viral oncogenes is a potential therapeutic strategy. The proof-of-concept work was done by the Wang group in 2014 using ZFNs against E7 DNA in HPV16/ 18-positive cervical cancer cells. Repressed xenograft formation was also evidenced in vivo (Ding et al., 2014b). Later, they reported similar work using the CRISPR/Cas system (Hu et al., 2014) and TALENs (Hu et al., 2015). Based on these promising preclinical data, four clinical trials were registered to study these approaches in humans (NCT02800369, NCT03057912, NCT03226470, NCT03057912).

The same strategy was also performed to remove other viruses, such as herpes simplex virus (HSV) or hepatitis B virus (HBV), from the genome. Recently, Yin et al. (2021) proved that intracorneal delivery of lentiviral particles encapsulating Cas9/sgRNA targeting HSV could not only cure herpetic stromal keratitis in a mouse disease model, but also prevent HSV infection in a prevention model. Their meaningful data also boosted the initiation of the corresponding clinical trial (NCT04560790). HBV is one of the most important pathogens responsible for liver diseases. The HBV genome exists as a double-stranded covalently closed circular DNA (cccDNA), which can be targeted by gene-editing tools. Expression of ZFN pairs targeting the HBV genome resulted in specific DNA cleavage and inhibition of active HBV replication (Cradick et al., 2010; Weber et al., 2014). A similar strategy was also applied using TALEN (Bloom et al., 2013; Chen et al., 2014). Recently, CRISPR/Cas9 was also employed to achieve highly efficient HBV genome elimination (Dong et al., 2015; Ramanan et al., 2015). Notably, Dong et al. (2015) showed that injection of sgRNACas9 plasmids via the tail vein reduced the levels of cccDNA and HBV protein in a mouse model carrying HBV cccDNA.

Another successful application of CRISPR/Cas for antiviral therapy was reported in 2014. Patient-derived cells from Burkitt's lymphoma patient with latent Epstein-Barr virus infection showed dramatic proliferation arrest and a concomitant decrease in viral load after exposure to a CRISPR/Cas9 vector targeted to the viral genome (Wang and Quake, 2014). All these studies indicate that disrupting viral genes using gene editing is a promising strategy to treat infectious diseases.

\section{Therapeutic gene editing: challenges and future directions}

Advances in gene-editing technologies have laid the foundation for next-generation therapies to cure a wide range of genetic and nongenetic diseases. However, great effort in diverse fields is still required to enable safe, effective and affordable clinical translation. Here, we provide an overview of the existing challenges and potential approaches to surmount them. We also discuss issues remaining to be addressed in the future to enable gene-editing technology to fulfill unmet biomedical needs.

\section{Challenges faced by current technologies}

Immune responses elicited by gene-editing tools could influence the therapeutic effects

As reviewed in the above sections, gene-editing tools can introduce double-stranded breaks and perform precise genetic modifications in the genome by removing, replacing, or adding pieces of DNA at targeted sites. It is worth 
highlighting that the majority of the enzymatic parts of these tools are derived from nonhuman species. For example, Cas proteins originate exclusively from bacteria, archaea or phages (Al-Shayeb et al., 2020; Makarova et al., 2020). Hence, the problem of immunity against exogenous proteins could not be avoided. On the one hand, preexisting neutralizing antibodies, along with antagonistic $\mathrm{T}$ cells against commonly used Cas nucleases, were found in the human body (Charlesworth et al., 2019; Wagner et al., 2019). Thus, the therapeutic efficacy may be compromised. Theoretically, these humoral and cellular immunity obstacles may be bypassed by exploiting new Cas orthologs from non-human-related microbes (Moreno et al., 2019) or by modifying the antigen epitope of the Cas proteins used if possible. On the other hand, adaptive immune responses against various components of CRISPR therapeutics could also be elicited. The effect of this problem is even more obvious when multiple dosing of gene-editing tools is needed. For example, Cas13-mediated RNA editing might require repeated dosing for long-lasting, effective transcriptional repression. In this situation, the therapeutic effects of the second dose or later doses could be impaired by the antibody produced following the first dose. In this case, using different Cas orthologs in each dosing to possibly evade adaptive immune responses might be one potential solution (Moreno et al., 2019).

\section{Current gene-editing technologies can elicit unintended DNA or RNA modifications}

The strong efficacy of existing gene-editing tools is usually accompanied by unintended DNA or RNA modifications. Taking the CRISPR system as an example, DNA recognition of the Cas-sgRNA complex cannot guarantee $100 \%$ specificity, as mismatches or other subtle changes in the target DNA can be tolerated (Hsu et al., 2013; Kleinstiver et al., 2016b; Strecker et al., 2019a; Tsai et al., 2015). In addition, scrambled trans-cleavage activity of Cas 12 or Cas 13 might occur following the cis-cleavage of targets (Abudayyeh et al., 2016; Chen et al., 2018; East-Seletsky et al., 2016). Other proteinic components, such as the deaminase of base editors, could also cause nonspecific genome- and transcriptomewide off-target deamination (Doman et al., 2020; Grünewald et al., 2019a; Grünewald et al., 2019b; Zhou et al., 2019a). More seriously, aside from off-target effects, nuclease-based gene editing can cause undesired on-target alterations in the genome, such as large elongated DNA deletions around the on-target DSBs (Adikusuma et al., 2018; Kosicki et al., 2018; Zuccaro et al., 2020) and changes to the chromosome structure, such as inversions or translocations (Frock et al., 2015; Maeder et al., 2019). To diminish these unintended gene editing outcomes, researchers have made great efforts to optimize these tools (Kim et al., 2019). However, to a certain degree, sacrificing on-target efficacy seems un- avoidable for engineered tools (Schmid-Burgk et al., 2020). Recently, one work showed that AsCas12a and LbCas12a induced no trans-cleavage off-target effects in mouse embryos, possibly due to the low concentration of genomic DNA in the cell nucleus (Wei et al., 2021). This result may partially ease our anxiety about the adverse effects of Cas nucleases and indicate that unintended gene editing in vivo might be distinct from that detected at the cellular level. In any case, systematically evaluating the gene-editing outcomes of the therapeutic cargo is required before clinical treatment. Importantly, the safety issues mentioned above are largely influenced by the delivery approach, which we will discuss in the next section.

An important therapeutic application related to off-target issues is performing allele-specific gene editing to treat heterozygous dominant genetic diseases. It is crucial to prevent off-target editing at normal gene sites while efficiently disrupting the pathogenic allele. A suitable PAM sequence may not be located near the mutated sites for DNA editing ( $\mathrm{Li}$ et al., 2018a). However, even when a canonical PAM exists, the mutations might not be enough for a 20 -nt spacer to distinguish the two alleles (Li et al., 2018a). The rational design of spacers may partially solve these problems, for example, by using truncated or tuned sgRNA ( $\mathrm{Li}$ et al., 2018a). A high-fidelity Cas enzyme to perfectly distinguish one-nucleotide difference between the wild-type and gain-of-function mutant allele is highly desired for this application. Nevertheless, this strategy is helpless when facing some complicated mutation types, for example, the expansion of repeated sequences (Rodriguez and Todd, 2019; Rudich and Lamitina, 2018). It seems that this problem may be addressed by RNA editing (Batra et al., 2017), as unproportioned RNA knockdown of transcripts from the two alleles can be accepted.

\section{In vivo delivery of gene-editing tools}

Benefiting from quality-controllable cell engineering with highly efficient biomacromolecule delivery methods such as electroporation, ex vivo genome editing has achieved impressive milestones in clinical trials (Esrick et al., 2021; Frangoul et al., 2021). However, compared with ex vivo gene editing, in vivo gene editing has advanced relatively slowly. One of the main contributing factors is the considerable complexity of our human body.

Therapeutic delivery vectors can be generally assigned into two distinct categories, viral and nonviral vector systems, according to their biological characteristics. AAV has become one of the most promising and commonly used viral vectors for gene therapy due to its high infection efficiency, broad tissue tropism, and low immunogenicity (Wang et al., 2019a; Wang et al., 2020a). However, the limited DNA packaging capability of AAV prevents the efficacious delivery of the commonly used SpCas9 nuclease and base and 
prime editors. Endeavors to discover smaller natural Cas nucleases or to evolve minimal Cas variants are an important need (Ran et al., 2015; Shams et al., 2021).

The wide application of AAV vectors for gene therapy also benefits from the resulting long-term gene expression. However, for gene-editing tools, transient expression is highly favorable because a long duration of efficacy can increase the safety risks mentioned above. From this perspective, it might be suboptimal to choose AAV to deliver gene-editing tools. However, a recent study reported that in vivo base editing in hepatocytes using SaCas9 KKH-CBE delivered by dual AAVs had no detectable off-target effects at either the RNA or DNA level (Villiger et al., 2021). The authors attributed this phenomenon to the low expression level of CBE in vivo due to the dual AAV strategy. Thus, it might be acceptable to carefully design the AAV vector to minimize the expression of its therapeutic cargo while maintaining its treatment efficacy.

On the other hand, nonviral vectors, especially nanoparticles, are highly suitable for the transient expression of the delivered cargos. A comprehensive summary of nonviral delivery strategies can be found in another review (Xu et al., 2021b). mRNAs commonly serve as therapeutic cargos for nanoparticles. The advantages of using nanoparticles to deliver mRNA-expressed gene-editing proteins are obvious. First, large base editors, prime editors, or multiple regulators can be introduced in mRNA format and packaged in nanoparticles to achieve efficient genome modifications (Song et al., 2020; Villiger et al., 2021). Moreover, some commonly used nanoparticles, such as lipid nanoparticles (LNPs), can be engineered with good manufacturing practices (GMPs) to ensure large-scale production and high quality (Kaczmarek et al., 2017). However, we should also note the limited tissue specificity of nanoparticles. Taking LNPs as an example, the predominant target site of most LNPs upon systemic delivery is usually restricted to the liver (Akinc et al., 2010). Although much effort has been made to engineer LNPs for broader tissue tropism (Samaridou et al., 2020), more information is needed on the mechanisms of the interactions between LNPs and targeted tissues.

The advantages of existing viral and nonviral vectors can be combined for an improved delivery strategy. One such strategy packaged mRNA in lentivirus virions (Ling et al., 2021). The authors further attached an MS2 recognition stem loop to the mRNA to mimic the package signal of the long terminal region (LTR), mediating an effective interaction with the capsid protein-fused MS2 protein. In this way, the high efficiency of transduction and transient expression of the two systems are combined together. This promising delivery method has been used to cure wet agerelated macular degeneration and virus-induced herpetic stromal keratitis in a mouse model (Ling et al., 2021; Yin et al., 2021).

\section{Future directions}

Thanks to today's powerful technologies, a major step forward was taken toward curing various diseases. However, patients still have unmet medical needs, which continues to drive scientists to develop novel gene-editing tools and therapeutic strategies. In this section, we will consider prospective future directions of gene-editing technology and discuss several tools with great potential to become novel therapeutic platforms.

Manipulating the human genome at a larger scale: correcting larger genomic variations

Nuclease-based genome editing may be used to correct mutations in many genetic diseases, especially HDR strategies, which can theoretically correct any genome alteration. In fact, this technology has been developing relatively slowly due to its extremely low efficiency in nonmitotic somatic cells (Cox et al., 2015; Zheng et al., 2014). New alternative tools, including base editors and prime editors, were developed to meet the need to correct mutations. These two editors can precisely and efficiently correct gene alterations such as point mutations, small genomic deletions, or insertions (<60 bp) (Anzalone et al., 2019; Gaudelli et al., 2017; Komor et al., 2016). However, it is very difficult for them to correct larger genome variations (>100 bp), such as genetic deletions, insertions, duplications, or inversions (insertions plus deletions), which cause many types of severe genetic diseases (Landrum et al., 2014). Although multiple guides can be employed to direct Cas nucleases to induce deletions or inversions of large DNA fragments (Maeder et al., 2019), undesired byproducts occur, increasing safety risks. It is worth noting that several works reported on bioRixv repurposed the prime editor to delete large DNA fragments using dual peg-sgRNAs (Choi et al., 2021; Jiang et al., 2021). However, the efficacy of this approach needs to be improved.

Accordingly, new technologies enabling manipulation of the human genome on a larger scale with high efficiency and precision are an important need. Here, we regard recombinase as a candidate, some types of which have already been widely applied in human cells to create large gene inversions and deletions with high efficiency and precision (Meinke et al., 2016; Van Duyne, 2015). One significant problem is that the recognizable DNA sequence for recombinase is highly restricted, making it difficult to apply to the natural human genome. In addition, engineerable sites on the human genome conforming to the canonical recognition model of recombinase are extremely limited (Lansing et al., 2020b), leaves little space for directed evolution or rational design. Fortunately, heterodimers of recombinases can be employed to recognize targets with asymmetric half-sites (Lansing et al., 2020b). Increasing the number of sites on the human genome can be chosen as templates for protein 
engineering. Encouragingly, this strategy has been used successfully to correct genomic inversions in a gene that encodes factor VIII causing hemophilia A in human cells (Lansing et al., 2020a).

A more general strategy for curing genetic diseases: from gene scissors to gene glue

Precisely correcting gene mutations in situ is ideal for curing genetic diseases. However, the dysfunction of one gene can be attributable to hundreds of different genetic alterations, which makes such personalized correction unaffordable in terms of time and expenses. Thus, developing a more general strategy to treat diseases caused by different genotypes is highly desirable. One elegant idea is to insert an extra copy of a gene in the human genome regardless of the existing aberrant one. A cDNA could be precisely integrated behind a normal endogenous gene, or a complete gene cassette could be inserted into safe harbors (Barzel et al., 2015; Wang et al., 2019b). It is unavoidable to refer again to HDR, which may have the ability to achieve this goal. However, as discussed above, the working mechanism of HDR relies heavily on the endogenous DNA repair machinery, which causes low efficiency in nonmitotic cells. Nevertheless, we should emphasize the availability of HDR when integrating gene fragments into the genomes of mitotic cells such as hepatocytes or stem cells (Barzel et al., 2015; Martin et al., 2019; Yin et al., 2014; Yin et al., 2016).

Transposase can integrate large DNA fragments into the genomes of diverse cell types with high efficiency (Hickman and Dyda, 2016; Ivics et al., 2009). In contrast to that of recombinase, the recognition sequence of the commonly used transposase is quite simple, which leads to random integration of the donor in the human genome. Thus, efforts should be made to restrict the function of transposase to unique sites in the human genome. One simple idea is to conjugate transposase with specific DNA recognition modules, but the efficiency and specificity of these systems remain to be further optimized (Feng et al., 2010; Kovač et al., 2020; Luo et al., 2017; Owens et al., 2012; Owens et al., 2013). In addition, we should note that the fused specific DNA recognition modules concentrated the transposase near the targeted sites, whereas the intact transposase domain still performed its genome-wide functions. This obstacle can potentially be addressed by two newly discovered CRISPR transposase systems: CRISPR-V-K and CRISPR-I-F (Klompe et al., 2019; Strecker et al., 2019b). Both of them enable efficient and programmable targeted integrations in bacterial genomes (Klompe et al., 2019; Strecker et al., 2019b; Vo et al., 2021). More work should be focused on elucidating the efficacy of these two systems in mammalian cells in the future.

Compliance and ethics The author(s) declare that they have no conflict of interest.

Acknowledgements This work was supported by the National Natural Science Foundation of China (81830004, 31922046, 31770057, 31722036, 31930016, 31870893), the Sanming Project of Medicine in Shenzhen (SZSM202011017), the National Key Research and Development Program of China (2018YFA0801401, 2019YFA0110800, 2018YFA0107703, 2019YFA0110000, 2020YFA0707800, 2020YFA0707600), the Strategic Priority Research Program of the Chinese Academy of Sciences (XDA16030403, XDA16010503), Beijing Municipal Science \& Technology Commission (Z181100001318009), Beijing Advanced Innovation Center for Genomics at Peking University and the Peking-Tsinghua Center for Life Sciences, and the National Major Science \& Technology Project for Control and Prevention of Major Infectious Diseases in China (2018ZX10301401). We are grateful to Dr. Gaofeng Fan from ShanghaiTech University for manuscript editing.

\section{References}

Abudayyeh, O.O., Gootenberg, J.S., Konermann, S., Joung, J., Slaymaker, I.M., Cox, D.B.T., Shmakov, S., Makarova, K.S., Semenova, E., Minakhin, L., et al. (2016). C2c2 is a single-component programmable RNA-guided RNA-targeting CRISPR effector. Science 353, aaf5573.

Abudayyeh, O.O., Gootenberg, J.S., Essletzbichler, P., Han, S., Joung, J., Belanto, J.J., Verdine, V., Cox, D.B.T., Kellner, M.J., Regev, A., et al. (2017). RNA targeting with CRISPR-Cas13. Nature 550, 280-284.

Abudayyeh, O.O., Gootenberg, J.S., Franklin, B., Koob, J., Kellner, M.J., Ladha, A., Joung, J., Kirchgatterer, P., Cox, D.B.T., and Zhang, F. (2019). A cytosine deaminase for programmable single-base RNA editing. Science 365, 382-386.

Ackerman, C.M., Myhrvold, C., Thakku, S.G., Freije, C.A., Metsky, H.C., Yang, D.K., Ye, S.H., Boehm, C.K., Kosoko-Thoroddsen, T.S.F., Kehe, J., et al. (2020). Massively multiplexed nucleic acid detection with Cas13. Nature 582, 277-282.

Adamson, B., Norman, T.M., Jost, M., Cho, M.Y., Nuñez, J.K., Chen, Y., Villalta, J.E., Gilbert, L.A., Horlbeck, M.A., Hein, M.Y., et al. (2016). A multiplexed single-cell CRISPR screening platform enables systematic dissection of the unfolded protein response. Cell 167, 1867-1882.e21.

Adikusuma, F., Piltz, S., Corbett, M.A., Turvey, M., McColl, S.R., Helbig, K.J., Beard, M.R., Hughes, J., Pomerantz, R.T., and Thomas, P.Q. (2018). Large deletions induced by Cas9 cleavage. Nature 560, E8-E9.

Aiuti, A., Slavin, S., Aker, M., Ficara, F., Deola, S., Mortellaro, A., Morecki, S., Andolfi, G., Tabucchi, A., Carlucci, F., et al. (2002). Correction of ADA-SCID by stem cell gene therapy combined with nonmyeloablative conditioning. Science 296, 2410-2413.

Aiuti, A., Cattaneo, F., Galimberti, S., Benninghoff, U., Cassani, B., Callegaro, L., Scaramuzza, S., Andolfi, G., Mirolo, M., Brigida, I., et al. (2009). Gene therapy for immunodeficiency due to adenosine deaminase deficiency. N Engl J Med 360, 447-458.

Akinc, A., Querbes, W., De, S., Qin, J., Frank-Kamenetsky, M., Jayaprakash, K.N., Jayaraman, M., Rajeev, K.G., Cantley, W.L., Dorkin, J.R., et al. (2010). Targeted delivery of RNAi therapeutics with endogenous and exogenous ligand-based mechanisms. Mol Ther 18, 1357-1364.

Al-Shayeb, B., Sachdeva, R., Chen, L.X., Ward, F., Munk, P., Devoto, A., Castelle, C.J., Olm, M.R., Bouma-Gregson, K., Amano, Y., et al. (2020). Clades of huge phages from across Earth's ecosystems. Nature 578, 425-431.

Allen, A.G., Chung, C.H., Atkins, A., Dampier, W., Khalili, K., Nonnemacher, M.R., and Wigdahl, B. (2018). Gene editing of HIV-1 co-receptors to prevent and/or cure virus infection. Front Microbiol 9, 2940.

Aman, R., Mahas, A., Marsic, T., Hassan, N., and Mahfouz, M.M. (2020). Efficient, rapid, and sensitive detection of plant RNA viruses with onepot RT-RPA-CRISPR/Cas12a assay. Front Microbiol 11, 610872.

Anders, C., Niewoehner, O., Duerst, A., and Jinek, M. (2014). Structural basis of PAM-dependent target DNA recognition by the Cas9 
endonuclease. Nature 513, 569-573.

Anzalone, A.V., Randolph, P.B., Davis, J.R., Sousa, A.A., Koblan, L.W., Levy, J.M., Chen, P.J., Wilson, C., Newby, G.A., Raguram, A., et al. (2019). Search-and-replace genome editing without double-strand breaks or donor DNA. Nature 576, 149-157.

Anzalone, A.V., Koblan, L.W., and Liu, D.R. (2020). Genome editing with CRISPR-Cas nucleases, base editors, transposases and prime editors. Nat Biotechnol 38, 824-844.

Auer, T.O., Duroure, K., De Cian, A., Concordet, J.P., and Del Bene, F. (2014). Highly efficient CRISPR/Cas9-mediated knock-in in zebrafish by homology-independent DNA repair. Genome Res 24, 142-153.

Bai, J., Lin, H., Li, H., Zhou, Y., Liu, J., Zhong, G., Wu, L., Jiang, W., Du, H., Yang, J., et al. (2019). Cas12a-based on-site and rapid nucleic acid detection of African swine fever. Front Microbiol 10, 2830.

Bainbridge, J.W.B., Smith, A.J., Barker, S.S., Robbie, S., Henderson, R., Balaggan, K., Viswanathan, A., Holder, G.E., Stockman, A., Tyler, N., et al. (2008). Effect of gene therapy on visual function in Leber's congenital amaurosis. N Engl J Med 358, 2231-2239.

Bainbridge, J.W.B., Mehat, M.S., Sundaram, V., Robbie, S.J., Barker, S.E., Ripamonti, C., Georgiadis, A., Mowat, F.M., Beattie, S.G., Gardner, P. J., et al. (2015). Long-term effect of gene therapy on Leber's congenital amaurosis. N Engl J Med 372, 1887-1897.

Bakondi, B., Lv, W., Lu, B., Jones, M.K., Tsai, Y., Kim, K.J., Levy, R., Akhtar, A.A., Breunig, J.J., Svendsen, C.N., et al. (2016). In vivo CRISPR/Cas9 gene editing corrects retinal dystrophy in the S334ter-3 rat model of autosomal dominant retinitis pigmentosa. Mol Ther 24 , 556-563.

Balderston, S., Taulbee, J.J., Celaya, E., Fung, K., Jiao, A., Smith, K., Hajian, R., Gasiunas, G., Kutanovas, S., Kim, D., et al. (2021). Discrimination of single-point mutations in unamplified genomic DNA via Cas9 immobilized on a graphene field-effect transistor. Nat Biomed Eng 5, 713-725.

Barrangou, R., Fremaux, C., Deveau, H..., Richards, M., Boyaval, P., Moineau, S., Romero, D.A., and Horvath, P. (2007). CRISPR provides acquired resistance against viruses in prokaryotes. Science 315, 1709 1712.

Barzel, A., Paulk, N.K., Shi, Y., Huang, Y., Chu, K., Zhang, F., Valdmanis, P.N., Spector, L.P., Porteus, M.H., Gaensler, K.M., et al. (2015). Promoterless gene targeting without nucleases ameliorates haemophilia B in mice. Nature 517, 360-364.

Batra, R., Nelles, D.A., Pirie, E., Blue, S.M., Marina, R.J., Wang, H., Chaim, I.A., Thomas, J.D., Zhang, N., Nguyen, V., et al. (2017). Elimination of toxic microsatellite repeat expansion RNA by RNAtargeting Cas9. Cell 170, 899-912.e10.

Beerli, R.R., and Barbas, C.F. (2002). Engineering polydactyl zinc-finger transcription factors. Nat Biotechnol 20, 135-141.

Benjamin, R., Graham, C., Yallop, D., Jozwik, A., Mirci-Danicar, O.C., Lucchini, G., Pinner, D., Jain, N., Kantarjian, H., Boissel, N., et al. (2020). Genome-edited, donor-derived allogeneic anti-CD19 chimeric antigen receptor $\mathrm{T}$ cells in paediatric and adult B-cell acute lymphoblastic leukaemia: results of two phase 1 studies. Lancet 396, 1885-1894.

Bennett, J., Ashtari, M., Wellman, J., Marshall, K.A., Cyckowski, L.L., Chung, D.C., McCague, S., Pierce, E.A., Chen, Y., Bennicelli, J.L., et al. (2012). AAV2 gene therapy readministration in three adults with congenital blindness. Sci Transl Med 4, 120 ra115.

Bennett, J., Wellman, J., Marshall, K.A., McCague, S., Ashtari, M., DiStefano-Pappas, J., Elci, O.U., Chung, D.C., Sun, J., Wright, J.F., et al. (2016). Safety and durability of effect of contralateral-eye administration of AAV2 gene therapy in patients with childhood-onset blindness caused by RPE65 mutations: a follow-on phase 1 trial. Lancet $388,661-672$.

Bibikova, M., Golic, M., Golic, K.G., and Carroll, D. (2002). Targeted chromosomal cleavage and mutagenesis in Drosophila using zinc-finger nucleases. Genetics 161, 1169-1175.

Billon, P., Bryant, E.E., Joseph, S.A., Nambiar, T.S., Hayward, S.B., Rothstein, R., and Ciccia, A. (2017). CRISPR-mediated base editing enables efficient disruption of eukaryotic genes through induction of STOP codons. Mol Cell 67, 1068-1079.e4.

Bjurström, C.F., Mojadidi, M., Phillips, J., Kuo, C., Lai, S., Lill, G.R., Cooper, A., Kaufman, M., Urbinati, F., Wang, X., et al. (2016). Reactivating fetal hemoglobin expression in human adult erythroblasts through BCL11A Knockdown Using Targeted Endonucleases. Mol Ther Nucleic Acids 5, e351.

Bloom, K., Ely, A., Mussolino, C., Cathomen, T., and Arbuthnot, P. (2013). Inactivation of hepatitis $\mathrm{B}$ virus replication in cultured cells and in vivo with engineered transcription activator-like effector nucleases. Mol Ther 21, 1889-1897.

Boch, J., and Bonas, U. (2010). Xanthomonas AvrBs3 family-type III effectors: discovery and function. Annu Rev Phytopathol 48, 419-436.

Boch, J., Scholze, H., Schornack, S., Landgraf, A., Hahn, S., Kay, S., Lahaye, T., Nickstadt, A., and Bonas, U. (2009). Breaking the code of DNA binding specificity of TAL-type III effectors. Science 326, 15091512.

Boettcher, M., Tian, R., Blau, J.A., Markegard, E., Wagner, R.T., Wu, D., Mo, X., Biton, A., Zaitlen, N., Fu, H., et al. (2018). Dual gene activation and knockout screen reveals directional dependencies in genetic networks. Nat Biotechnol 36, 170-178.

Bolotin, A., Quinquis, B., Sorokin, A., and Ehrlich, S.D. (2005). Clustered regularly interspaced short palindrome repeats (CRISPRs) have spacers of extrachromosomal origin. Microbiology 151, 2551-2561.

Boye, S.E., Huang, W.C., Roman, A.J., Sumaroka, A., Boye, S.L., Ryals, R. C., Olivares, M.B., Ruan, Q., Tucker, B.A., Stone, E.M., et al. (2014). Natural history of cone disease in the murine model of Leber congenital amaurosis due to CEP290 mutation: determining the timing and expectation of therapy. PLoS ONE 9, e92928.

Broughton, J.P., Deng, X., Yu, G., Fasching, C.L., Servellita, V., Singh, J., Miao, X., Streithorst, J.A., Granados, A., Sotomayor-Gonzalez, A., et al. (2020). CRISPR-Cas12-based detection of SARS-CoV-2. Nat Biotechnol 38, 870-874.

Burr, M.L., Sparbier, C.E., Chan, Y.C., Williamson, J.C., Woods, K., Beavis, P.A., Lam, E.Y.N., Henderson, M.A., Bell, C.C., Stolzenburg, S., et al. (2017). CMTM6 maintains the expression of PD-L1 and regulates anti-tumour immunity. Nature 549, 101-105.

Butler, J.R., Martens, G.R., Estrada, J.L., Reyes, L.M., Ladowski, J.M., Galli, C., Perota, A., Cunningham, C.M., Tector, M., and Joseph Tector, A. (2016). Silencing porcine genes significantly reduces human-anti-pig cytotoxicity profiles: an alternative to direct complement regulation. Transgenic Res 25, 751-759.

Cai, P., Otten, A.B.C., Cheng, B., Ishii, M.A., Zhang, W., Huang, B., Qu, K., and Sun, B.K. (2020). A genome-wide long noncoding RNA CRISPRi screen identifies PRANCR as a novel regulator of epidermal homeostasis. Genome Res 30, 22-34.

Cai, Y., Cheng, T., Yao, Y., Li, X., Ma, Y., Li, L., Zhao, H., Bao, J., Zhang, M., Qiu, Z., et al. (2019). In vivo genome editing rescues photoreceptor degeneration via a Cas9/RecA-mediated homology-directed repair pathway. Sci Adv 5, eaav3335.

Canver, M.C., Smith, E.C., Sher, F., Pinello, L., Sanjana, N.E., Shalem, O., Chen, D.D., Schupp, P.G., Vinjamur, D.S., Garcia, S.P., et al. (2015). BCL11A enhancer dissection by Cas9-mediated in situ saturating mutagenesis. Nature 527, 192-197.

Carreras, A., Pane, L.S., Nitsch, R., Madeyski-Bengtson, K., Porritt, M., Akcakaya, P., Taheri-Ghahfarokhi, A., Ericson, E., Bjursell, M., PerezAlcazar, M., et al. (2019). In vivo genome and base editing of a human PCSK9 knock-in hypercholesterolemic mouse model. BMC Biol 17, 4.

Carroll, K.J., Makarewich, C.A., McAnally, J., Anderson, D.M., Zentilin, L., Liu, N., Giacca, M., Bassel-Duby, R., and Olson, E.N. (2016). A mouse model for adult cardiac-specific gene deletion with CRISPR/ Cas9. Proc Natl Acad Sci USA 113, 338-343.

Ceccaldi, R., Rondinelli, B., and D'Andrea, A.D. (2016). Repair pathway choices and consequences at the double-strand break. Trends Cell Biol $26,52-64$.

Cermak, T., Doyle, E.L., Christian, M., Wang, L., Zhang, Y., Schmidt, C., Baller, J.A., Somia, N.V., Bogdanove, A.J., and Voytas, D.F. (2011). 
Efficient design and assembly of custom TALEN and other TAL effector-based constructs for DNA targeting. Nucleic Acids Res 39, e82.

Chadwick, A.C., Wang, X., and Musunuru, K. (2017). In vivo base editing of PCSK9 (proprotein convertase subtilisin/kexin type 9) as a therapeutic alternative to genome editing. Arterioscler Thromb Vasc Biol 37, 1741-1747.

Chang, N., Sun, C., Gao, L., Zhu, D., Xu, X., Zhu, X., Xiong, J.W., and Xi, J.J. (2013). Genome editing with RNA-guided Cas9 nuclease in zebrafish embryos. Cell Res 23, 465-472.

Charlesworth, C.T., Deshpande, P.S., Dever, D.P., Camarena, J., Lemgart, V.T., Cromer, M.K., Vakulskas, C.A., Collingwood, M.A., Zhang, L., Bode, N.M., et al. (2019). Identification of preexisting adaptive immunity to Cas9 proteins in humans. Nat Med 25, 249-254.

Chen, H., Shi, M., Gilam, A., Zheng, Q., Zhang, Y., Afrikanova, I., Li, J., Gluzman, Z., Jiang, R., Kong, L.J., et al. (2019a). Hemophilia A ameliorated in mice by CRISPR-based in vivo genome editing of human factor VIII. Sci Rep 9, 16838.

Chen, J., Yang, B., and Yang, L. (2019b). To BE or not to BE, that is the question. Nat Biotechnol 37, 520-522.

Chen, J., Zhang, W., Lin, J., Wang, F., Wu, M., Chen, C., Zheng, Y., Peng, X., Li, J., and Yuan, Z. (2014). An efficient antiviral strategy for targeting hepatitis $\mathrm{B}$ virus genome using transcription activator-like effector nucleases. Mol Ther 22, 303-311.

Chen, J.S., Dagdas, Y.S., Kleinstiver, B.P., Welch, M.M., Sousa, A.A., Harrington, L.B., Sternberg, S.H., Joung, J.K., Yildiz, A., and Doudna, J.A. (2017a). Enhanced proofreading governs CRISPR-Cas9 targeting accuracy. Nature 550, 407-410.

Chen, J.S., Ma, E., Harrington, L.B., Da Costa, M., Tian, X., Palefsky, J.M., and Doudna, J.A. (2018). CRISPR-Cas12a target binding unleashes indiscriminate single-stranded DNase activity. Science 360, 436-439.

Chen, S., Sanjana, N.E., Zheng, K., Shalem, O., Lee, K., Shi, X., Scott, D. A., Song, J., Pan, J.Q., Weissleder, R., et al. (2015a). Genome-wide CRISPR screen in a mouse model of tumor growth and metastasis. Cell 160, 1246-1260.

Chen, Y., Zheng, Y., Kang, Y., Yang, W., Niu, Y., Guo, X., Tu, Z., Si, C., Wang, H., Xing, R., et al. (2015b). Functional disruption of the dystrophin gene in rhesus monkey using CRISPR/Cas9. Hum Mol Genet 24, 3764-3774.

Chen, Y., Niu, Y., and Ji, W. (2016). Genome editing in nonhuman primates: approach to generating human disease models. J Intern Med 280, 246-251.

Chen, Y., Yu, J., Niu, Y., Qin, D., Liu, H., Li, G., Hu, Y., Wang, J., Lu, Y., Kang, Y., et al. (2017b). Modeling rett syndrome using TALEN-edited MECP2 mutant cynomolgus monkeys. Cell 169, 945-955.e10.

Chen, Z., Arai, E., Khan, O., Zhang, Z., Ngiow, S.F., He, Y., Huang, H., Manne, S., Cao, Z., Baxter, A.E., et al. (2021). In vivo $\mathrm{CD}^{+}{ }^{\mathrm{T}}$ cell CRISPR screening reveals control by Fli1 in infection and cancer. Cell 184, 1262-1280.e22.

Cheng, L., Li, Y., Qi, Q., Xu, P., Feng, R., Palmer, L., Chen, J., Wu, R., Yee, T., Zhang, J., et al. (2021). Single-nucleotide-level mapping of DNA regulatory elements that control fetal hemoglobin expression. Nat Genet 53, 869-880.

Chertow, D.S. (2018). Next-generation diagnostics with CRISPR. Science 360, 381-382.

Chesselet, M.F., and Carmichael, S.T. (2012). Animal models of neurological disorders. Neurotherapeutics 9, 241-244.

Chiu, W., Lin, T.Y., Chang, Y.C., Isahwan-Ahmad Mulyadi Lai, H., Lin, S. C., Ma, C., Yarmishyn, A.A., Lin, S.C., Chang, K.J., Chou, Y.B., et al. (2021). An update on gene therapy for inherited retinal dystrophy: experience in Leber congenital amaurosis clinical trials. Int J Mol Sci 22,4534

Choi, J., Chen, W., Suiter, C.C., Lee, C., Chardon, F.M., Yang, W., Leith, A., Daza, R.M., Martin, B., and Shendure, J. (2021). Precise genomic deletions using paired prime editing. Nat Biotechnol doi: 10.1038/ s41587-021-01025-z.

Choo, Y., Sánchez-García, I., and Klug, A. (1994). In vivo repression by a site-specific DNA-binding protein designed against an oncogenic sequence. Nature 372, 642-645.

Choong, C.J., Baba, K., and Mochizuki, H. (2016). Gene therapy for neurological disorders. Expert Opin Biol Ther 16, 143-159.

Chow, R.D., and Chen, S. (2018). Cancer CRISPR screens in vivo. Trends Cancer 4, 349-358.

Chow, R.D., Guzman, C.D., Wang, G., Schmidt, F., Youngblood, M.W., Ye, L., Errami, Y., Dong, M.B., Martinez, M.A., Zhang, S., et al. (2017). AAV-mediated direct in vivo CRISPR screen identifies functional suppressors in glioblastoma. Nat Neurosci 20, 1329-1341.

Christian, M., Cermak, T., Doyle, E.L., Schmidt, C., Zhang, F., Hummel, A., Bogdanove, A.J., and Voytas, D.F. (2010). Targeting DNA doublestrand breaks with TAL effector nucleases. Genetics 186, 757-761.

Cibelli, J.B., and Gurdon, J.B. (2018). Custom-made oocytes to clone nonhuman primates. Cell 172, 647-649.

Cideciyan, A.V., Hauswirth, W.W., Aleman, T.S., Kaushal, S., Schwartz, S. B., Boye, S.L., Windsor, E.A.M., Conlon, T.J., Sumaroka, A., Pang, J.J., et al. (2009). Human RPE65 gene therapy for Leber congenital amaurosis: persistence of early visual improvements and safety at 1 year. Hum Gene Ther 20, 999-1004.

Cimprich, K.A., and Cortez, D. (2008). ATR: an essential regulator of genome integrity. Nat Rev Mol Cell Biol 9, 616-627.

Cong, L., Ran, F.A., Cox, D., Lin, S., Barretto, R., Habib, N., Hsu, P.D., Wu, X., Jiang, W., Marraffini, L.A., et al. (2013). Multiplex genome engineering using CRISPR/Cas systems. Science 339, 819-823.

Conway, A., Mendel, M., Kim, K., McGovern, K., Boyko, A., Zhang, L., Miller, J.C., DeKelver, R.C., Paschon, D.E., Mui, B.L., et al. (2019). Non-viral delivery of zinc finger nuclease mRNA enables highly efficient in vivo genome editing of multiple therapeutic gene targets. Mol Ther 27, 866-877.

Cooper, M.L., Choi, J., Staser, K., Ritchey, J.K., Devenport, J.M., Eckardt, K., Rettig, M.P., Wang, B., Eissenberg, L.G., Ghobadi, A., et al. (2018). An "off-the-shelf" fratricide-resistant CAR-T for the treatment of T cell hematologic malignancies. Leukemia 32, 1970-1983.

Corman, V.M., Landt, O., Kaiser, M., Molenkamp, R., Meijer, A., Chu, D. K., Bleicker, T., Brünink, S., Schneider, J., Schmidt, M.L., et al. (2020). Detection of 2019 novel coronavirus (2019-nCoV) by real-time RTPCR. Eurosurveillance 25.

Cortez, J.T., Montauti, E., Shifrut, E., Gatchalian, J., Zhang, Y., Shaked, O., Xu, Y., Roth, T.L., Simeonov, D.R., Zhang, Y., et al. (2020). CRISPR screen in regulatory $\mathrm{T}$ cells reveals modulators of Foxp3. Nature 582, 416-420.

Cox, D.B.T., Platt, R.J., and Zhang, F. (2015). Therapeutic genome editing: prospects and challenges. Nat Med 21, 121-131.

Cox, D.B.T., Gootenberg, J.S., Abudayyeh, O.O., Franklin, B., Kellner, M. J., Joung, J., and Zhang, F. (2017). RNA editing with CRISPR-Cas13. Science 358, 1019-1027.

Cradick, T.J., Keck, K., Bradshaw, S., Jamieson, A.C., and McCaffrey, A.P. (2010). Zinc-finger nucleases as a novel therapeutic strategy for targeting hepatitis B virus DNAs. Mol Ther 18, 947-954.

Crosby, J., Peloso, G.M., Auer, P.L., Crosslin, D.R., Stitziel, N.O., Lange, L.A., Lu, Y., Tang, Z., Zhang, H., Hindy, G., et al. (2014). Loss-offunction mutations in $A P O C 3$, triglycerides, and coronary disease. $\mathrm{N}$ Engl J Med 371, 22-31.

Cuella-Martin, R., Hayward, S.B., Fan, X., Chen, X., Huang, J.W., Taglialatela, A., Leuzzi, G., Zhao, J., Rabadan, R., Lu, C., et al. (2021). Functional interrogation of DNA damage response variants with base editing screens. Cell 184, 1081-1097.e19.

Cui, C., Liu, Y., Gerloff, D., Rohde, C., Pauli, C., Köhn, M., Misiak, D., Oellerich, T., Schwartz, S., Schmidt, L.H., et al. (2021). NOP10 predicts lung cancer prognosis and its associated small nucleolar RNAs drive proliferation and migration. Oncogene 40, 909-921.

Cyranoski, D. (2018). First monkeys cloned with technique that made Dolly the sheep. Nature 553, 387-388.

Czernik, M., Anzalone, D.A., Palazzese, L., Oikawa, M., and Loi, P. (2019). Somatic cell nuclear transfer: failures, successes and the challenges ahead. Int J Dev Biol 63, 123-130. 
Dai, Y., Somoza, R.A., Wang, L., Welter, J.F., Li, Y., Caplan, A.I., and Liu, C.C. (2019). Exploring the trans-cleavage activity of CRISPR-Cas12a (cpf1) for the development of a universal electrochemical biosensor. Angew Chem Int Ed 58, 17399-17405.

Daponte, V., Tylzanowski, P., and Forlino, A. (2021). Appendage regeneration in vertebrates: what makes this possible? Cells 10, 242.

den Hollander, A.I., Koenekoop, R.K., Yzer, S., Lopez, I., Arends, M.L., Voesenek, K.E.J., Zonneveld, M.N., Strom, T.M., Meitinger, T., Brunner, H.G., et al. (2006). Mutations in the CEP290 (NPHP6) gene are a frequent cause of Leber congenital amaurosis. Am J Hum Genet 79, 556-561.

Deng, W., Shi, X., Tjian, R., Lionnet, T., and Singer, R.H. (2015). CASFISH: CRISPR/Cas9-mediated in situ labeling of genomic loci in fixed cells. Proc Natl Acad Sci USA 112, 11870-11875.

Deriano, L., and Roth, D.B. (2013). Modernizing the nonhomologous endjoining repertoire: alternative and classical NHEJ share the stage. Annu Rev Genet 47, 433-455.

Dersh, D., Phelan, J.D., Gumina, M.E., Wang, B., Arbuckle, J.H., Holly, J., Kishton, R.J., Markowitz, T.E., Seedhom, M.O., Fridlyand, N., et al. (2021). Genome-wide screens identify lineage- and tumor-specific genes modulating MHC-I- and MHC-II-restricted immunosurveillance of human lymphomas. Immunity 54, 116-131.e10.

Dever, D.P., Bak, R.O., Reinisch, A., Camarena, J., Washington, G., Nicolas, C.E., Pavel-Dinu, M., Saxena, N., Wilkens, A.B., Mantri, S., et al. (2016). CRISPR/Cas9 $\beta$-globin gene targeting in human haematopoietic stem cells. Nature 539, 384-389.

Diao, Y., Li, B., Meng, Z., Jung, I., Lee, A.Y., Dixon, J., Maliskova, L., Guan, K.L., Shen, Y., and Ren, B. (2016). A new class of temporarily phenotypic enhancers identified by CRISPR/Cas9-mediated genetic screening. Genome Res 26, 397-405.

Diao, Y., Fang, R., Li, B., Meng, Z., Yu, J., Qiu, Y., Lin, K.C., Huang, H., Liu, T., Marina, R.J., et al. (2017). A tiling-deletion-based genetic screen for cis-regulatory element identification in mammalian cells. Nat Methods 14, 629-635.

Didigu, C.A., Wilen, C.B., Wang, J., Duong, J., Secreto, A.J., DanetDesnoyers, G.A., Riley, J.L., Gregory, P.D., June, C.H., Holmes, M.C., et al. (2014). Simultaneous zinc-finger nuclease editing of the HIV coreceptors $c c r 5$ and $c x c r 4$ protects $\mathrm{CD}^{+}{ }^{+} \mathrm{T}$ cells from HIV-1 infection. Blood 123, 61-69.

Ding, Q., Strong, A., Patel, K.M., Ng, S.L., Gosis, B.S., Regan, S.N., Cowan, C.A., Rader, D.J., and Musunuru, K. (2014a). Permanent alteration of PCSK9 with in vivo CRISPR-Cas9 genome editing. Circ Res 115, 488-492.

Ding, W., Hu, Z., Zhu, D., Jiang, X., Yu, L., Wang, X., Zhang, C., Wang, L., Ji, T., Li, K., et al. (2014b). Zinc finger nucleases targeting the human papillomavirus $E 7$ oncogene induce $E 7$ disruption and a transformed phenotype in HPV16/18-positive cervical cancer cells. Clin Cancer Res 20, 6495-6503.

Ding, X., Yin, K., Li, Z., Lalla, R.V., Ballesteros, E., Sfeir, M.M., and Liu, C. (2020). Ultrasensitive and visual detection of SARS-CoV-2 using allin-one dual CRISPR-Cas12a assay. Nat Commun 11, 4711.

Dixit, A., Parnas, O., Li, B., Chen, J., Fulco, C.P., Jerby-Arnon, L., Marjanovic, N.D., Dionne, D., Burks, T., Raychowdhury, R., et al. (2016). Perturb-Seq: dissecting molecular circuits with scalable singlecell RNA profiling of pooled genetic screens. Cell 167, 1853-1866.e17.

Doman, J.L., Raguram, A., Newby, G.A., and Liu, D.R. (2020). Evaluation and minimization of Cas9-independent off-target DNA editing by cytosine base editors. Nat Biotechnol 38, 620-628.

Dong, C., Qu, L., Wang, H., Wei, L., Dong, Y., and Xiong, S. (2015). Targeting hepatitis B virus cccDNA by CRISPR/Cas9 nuclease efficiently inhibits viral replication. Antiviral Res 118, 110-117.

Dong, M.B., Wang, G., Chow, R.D., Ye, L., Zhu, L., Dai, X., Park, J.J., Kim, H.R., Errami, Y., Guzman, C.D., et al. (2019). Systematic immunotherapy target discovery using genome-scale in vivo CRISPR screens in CD8 T cells. Cell 178, 1189-1204.e23.

Dow, L.E., and Lowe, S.W. (2012). Life in the fast lane: mammalian disease models in the genomics era. Cell 148, 1099-1109.
Dow, L.E., Fisher, J., O’Rourke, K.P., Muley, A., Kastenhuber, E.R., Livshits, G., Tschaharganeh, D.F., Socci, N.D., and Lowe, S.W. (2015). Inducible in vivo genome editing with CRISPR-Cas9. Nat Biotechnol 33, 390-394.

Dunbar, C.E., High, K.A., Joung, J.K., Kohn, D.B., Ozawa, K., and Sadelain, M. (2018). Gene therapy comes of age. Science 359.

East-Seletsky, A., O'Connell, M.R., Knight, S.C., Burstein, D., Cate, J.H. D., Tjian, R., and Doudna, J.A. (2016). Two distinct RNase activities of CRISPR-C2c2 enable guide-RNA processing and RNA detection. Nature 538, 270-273.

Ebbesen, K.K., Kjems, J., and Hansen, T.B. (2016). Circular RNAs: identification, biogenesis and function. Biochim Biophys Acta 1859, $163-168$.

Esrick, E.B., Lehmann, L.E., Biffi, A., Achebe, M., Brendel, C., Ciuculescu, M.F., Daley, H., MacKinnon, B., Morris, E., Federico, A., et al. (2021). Post-transcriptional genetic silencing of BCL11A to treat sickle cell disease. N Engl J Med 384, 205-215.

Feng, X., Bednarz, A.L., and Colloms, S.D. (2010). Precise targeted integration by a chimaeric transposase zinc-finger fusion protein. Nucleic Acids Res 38, 1204-1216.

Fischer, K., Rieblinger, B., Hein, R., Sfriso, R., Zuber, J., Fischer, A., Klinger, B., Liang, W., Flisikowski, K., Kurome, M., et al. (2020). Viable pigs after simultaneous inactivation of porcine MHC class I and three xenoreactive antigen genes GGTA1, CMAH and B4GALNT2. Xenotransplantation 27.

Fozouni, P., Son, S., Díaz de León Derby, M., Knott, G.J., Gray, C.N., D'Ambrosio, M.V., Zhao, C., Switz, N.A., Kumar, G.R., Stephens, S.I., et al. (2021). Amplification-free detection of SARS-CoV-2 with CRISPR-Cas13a and mobile phone microscopy. Cell 184, 323-333.e9.

Frangoul, H., Altshuler, D., Cappellini, M.D., Chen, Y.S., Domm, J., Eustace, B.K., Foell, J., de la Fuente, J., Grupp, S., Handgretinger, R., et al. (2021). CRISPR-Cas9 gene editing for sickle cell disease and $\beta$ thalassemia. N Engl J Med 384, 252-260.

Frock, R.L., Hu, J., Meyers, R.M., Ho, Y.J., Kii, E., and Alt, F.W. (2015). Genome-wide detection of DNA double-stranded breaks induced by engineered nucleases. Nat Biotechnol 33, 179-186.

Fu, R., Fang, M., Xu, K., Ren, J., Zou, J., Su, L., Chen, X., An, P.P., Yu, D., $\mathrm{Ka}$, M., et al. (2020). Generation of $G G T A 1^{-/-} \beta 2 \mathrm{M}^{-1} \mathrm{CIITA}^{-/-}$pigs using CRISPR/Cas9 technology to alleviate xenogeneic immune reactions. Transplantation 104, 1566-1573.

Fu, Y., Sander, J.D., Reyon, D., Cascio, V.M., and Joung, J.K. (2014). Improving CRISPR-Cas nuclease specificity using truncated guide RNAs. Nat Biotechnol 32, 279-284.

Fulco, C.P., Munschauer, M., Anyoha, R., Munson, G., Grossman, S.R., Perez, E.M., Kane, M., Cleary, B., Lander, E.S., and Engreitz, J.M. (2016). Systematic mapping of functional enhancer-promoter connections with CRISPR interference. Science 354, 769-773.

Ganbaatar, U., and Liu, C. (2021). CRISPR-based COVID-19 testing: toward next-generation point-of-care diagnostics. Front Cell Infect Microbiol 11, 663949.

Gao, X., Tao, Y., Lamas, V., Huang, M., Yeh, W.H., Pan, B., Hu, Y.J., Hu, J. H., Thompson, D.B., Shu, Y., et al. (2018). Treatment of autosomal dominant hearing loss by in vivo delivery of genome editing agents. Nature 553, 217-221.

Garrelfs, S.F., Frishberg, Y., Hulton, S.A., Koren, M.J., O'Riordan, W.D., Cochat, P., Deschênes, G., Shasha-Lavsky, H., Saland, J.M., Van’t Hoff, W.G., et al. (2021). Lumasiran, an RNAi therapeutic for primary hyperoxaluria type 1. N Engl J Med 384, 1216-1226.

Gasiunas, G., Barrangou, R., Horvath, P., and Siksnys, V. (2012). Cas9crRNA ribonucleoprotein complex mediates specific DNA cleavage for adaptive immunity in bacteria. Proc Natl Acad Sci USA 109, E2579E2586.

Gasperini, M., Findlay, G.M., McKenna, A., Milbank, J.H., Lee, C., Zhang, M.D., Cusanovich, D.A., and Shendure, J. (2017). CRISPR/Cas9mediated scanning for regulatory elements required for HPRT1 expression via thousands of large, programmed genomic deletions. Am J Hum Genet 101, 192-205. 
Gaudelli, N.M., Komor, A.C., Rees, H.A., Packer, M.S., Badran, A.H., Bryson, D.I., and Liu, D.R. (2017). Programmable base editing of A $\bullet T$ to $\mathrm{G} \cdot \mathrm{C}$ in genomic DNA without DNA cleavage. Nature $551,464-471$.

Giannelli, S.G., Luoni, M., Castoldi, V., Massimino, L., Cabassi, T., Angeloni, D., Demontis, G.C., Leocani, L., Andreazzoli, M., and Broccoli, V. (2018). Cas9/sgRNA selective targeting of the P23H Rhodopsin mutant allele for treating retinitis pigmentosa by intravitreal AAV9.PHP.B-based delivery. Hum Mol Genet 27, 761-779.

Gilbert, L.A., Larson, M.H., Morsut, L., Liu, Z., Brar, G.A., Torres, S.E., Stern-Ginossar, N., Brandman, O., Whitehead, E.H., Doudna, J.A., et al. (2013). CRISPR-mediated modular RNA-guided regulation of transcription in eukaryotes. Cell 154, 442-451.

Gillmore, J.D., Gane, E., Taubel, J., Kao, J., Fontana, M., Maitland, M.L., Seitzer, J., O'Connell, D., Walsh, K.R., Wood, K., et al. (2021). CRISPR-Cas9 in vivo gene editing for transthyretin amyloidosis. N Engl J Med 385, 493-502.

Goh, C.J.H., Wong, J.H., El Farran, C., Tan, B.X., Coffill, C.R., Loh, Y.H., Lane, D., and Arumugam, P. (2021). Identification of pathways modulating vemurafenib resistance in melanoma cells via a genomewide CRISPR/Cas9 screen. G3 11.

Gootenberg, J.S., Abudayyeh, O.O., Kellner, M.J., Joung, J., Collins, J.J., and Zhang, F. (2018). Multiplexed and portable nucleic acid detection platform with Cas13, Cas12a, and Csm6. Science 360, 439-444.

Gootenberg, J.S., Abudayyeh, O.O., Lee, J.W., Essletzbichler, P., Dy, A.J., Joung, J., Verdine, V., Donghia, N., Daringer, N.M., Freije, C.A., et al. (2017). Nucleic acid detection with CRISPR-Cas13a/C2c2. Science $356,438-442$.

Grünewald, J., Zhou, R., Garcia, S.P., Iyer, S., Lareau, C.A., Aryee, M.J., and Joung, J.K. (2019a). Transcriptome-wide off-target RNA editing induced by CRISPR-guided DNA base editors. Nature 569, 433-437.

Grünewald, J., Zhou, R., Iyer, S., Lareau, C.A., Garcia, S.P., Aryee, M.J., and Joung, J.K. (2019b). CRISPR DNA base editors with reduced RNA off-target and self-editing activities. Nat Biotechnol 37, 1041-1048.

Guan, Y., Ma, Y., Li, Q., Sun, Z., Ma, L., Wu, L., Wang, L., Zeng, L., Shao, Y., Chen, Y., et al. (2016). CRISPR/Cas9-mediated somatic correction of a novel coagulator factor ${ }^{\mathrm{IX}}$ gene mutation ameliorates hemophilia in mouse. EMBO Mol Med 8, 477-488.

Guilinger, J.P., Thompson, D.B., and Liu, D.R. (2014). Fusion of catalytically inactive Cas9 to FokI nuclease improves the specificity of genome modification. Nat Biotechnol 32, 577-582.

Guo, M., Xu, Y., Dong, Z., Zhou, Z., Cong, N., Gao, M., Huang, W., Wang, Y., Liu, G., and Xian, X. (2020a). Inactivation of ApoC3 by CRISPR/ Cas9 protects against atherosclerosis in hamsters. Circ Res 127, 14561458.

Guo, Q., Feng, X., and Zhou, Y. (2020b). PCSK9 variants in familial hypercholesterolemia: a comprehensive synopsis. Front Genet 11, 1020.

Guo, S., Chen, Y., Liu, J., Zhang, X., Liu, Z., Zhou, Z., and Wei, W. (2022). Low-density lipoprotein receptor-related protein 1 is a CROPsassociated receptor for Clostridioides infection toxin B. Sci China Life Sci 65, 107-118.

Gurumurthy, C.B., O’Brien, A.R., Quadros, R.M., Adams Jr, J., Alcaide, P., Ayabe, S., Ballard, J., Batra, S.K., Beauchamp, M.C., Becker, K.A., et al. (2019). Reproducibility of CRISPR-Cas9 methods for generation of conditional mouse alleles: a multi-center evaluation. Genome Biol 20, 171.

György, B., Nist-Lund, C., Pan, B., Asai, Y., Karavitaki, K.D., Kleinstiver, B.P., Garcia, S.P., Zaborowski, M.P., Solanes, P., Spataro, S., et al. (2019). Allele-specific gene editing prevents deafness in a model of dominant progressive hearing loss. Nat Med 25, 1123-1130.

Haapaniemi, E., Botla, S., Persson, J., Schmierer, B., and Taipale, J. (2018). CRISPR-Cas9 genome editing induces a p53-mediated DNA damage response. Nat Med 24, 927-930.

Hacein-Bey-Abina, S., Garrigue, A., Wang, G.P., Soulier, J., Lim, A., Morillon, E., Clappier, E., Caccavelli, L., Delabesse, E., Beldjord, K., et al. (2008). Insertional oncogenesis in 4 patients after retrovirusmediated gene therapy of SCID-X1. J Clin Invest 118, 3132-3142.

Hajian, R., Balderston, S., Tran, T., deBoer, T., Etienne, J., Sandhu, M.,
Wauford, N.A., Chung, J.Y., Nokes, J., Athaiya, M., et al. (2019). Detection of unamplified target genes via CRISPR-Cas9 immobilized on a graphene field-effect transistor. Nat Biomed Eng 3, 427-437.

Han, K., Jeng, E.E., Hess, G.T., Morgens, D.W., Li, A., and Bassik, M.C. (2017). Synergistic drug combinations for cancer identified in a CRISPR screen for pairwise genetic interactions. Nat Biotechnol 35, 463-474.

Hanna, R.E., Hegde, M., Fagre, C.R., DeWeirdt, P.C., Sangree, A.K., Szegletes, Z., Griffith, A., Feeley, M.N., Sanson, K.R., Baidi, Y., et al. (2021). Massively parallel assessment of human variants with base editor screens. Cell 184, 1064-1080.e20.

Harel, I., Benayoun, B.A., Machado, B., Singh, P.P., Hu, C.K., Pech, M.F., Valenzano, D.R., Zhang, E., Sharp, S.C., Artandi, S.E., et al. (2015). A platform for rapid exploration of aging and diseases in a naturally shortlived vertebrate. Cell 160, 1013-1026.

Harrington, L.B., Burstein, D., Chen, J.S., Paez-Espino, D., Ma, E., Witte, I.P., Cofsky, J.C., Kyrpides, N.C., Banfield, J.F., and Doudna, J.A. (2018). Programmed DNA destruction by miniature CRISPR-Cas 14 enzymes. Science 362, 839-842.

Harris, R.S., and Liddament, M.T. (2004). Retroviral restriction by APOBEC proteins. Nat Rev Immunol 4, 868-877.

He, J., Hu, P., Gao, Y., Zheng, S., Xu, C., Liu, R., Fang, L., Li, R., Han, C., An, J., et al. (2020). Comparison and application of different immunoassay methods for the detection of SARS-CoV-2. J Med Virol 92, 2777-2784.

Heianza, Y., and Qi, L. (2019). Impact of genes and environment on obesity and cardiovascular disease. Endocrinology 160, 81-100.

Hendel, A., Bak, R.O., Clark, J.T., Kennedy, A.B., Ryan, D.E., Roy, S., Steinfeld, I., Lunstad, B.D., Kaiser, R.J., Wilkens, A.B., et al. (2015). Chemically modified guide RNAs enhance CRISPR-Cas genome editing in human primary cells. Nat Biotechnol 33, 985-989.

Hess, G.T., Frésard, L., Han, K., Lee, C.H., Li, A., Cimprich, K.A., Montgomery, S.B., and Bassik, M.C. (2016). Directed evolution using dCas9-targeted somatic hypermutation in mammalian cells. Nat Methods 13, 1036-1042.

Hickman, A.B., and Dyda, F. (2016). DNA transposition at work. Chem Rev 116, 12758-12784.

Holme, E., and Lindstedt, S. (1998). Tyrosinaemia type I and NTBC (2-(2nitro-4-trifluoromethylbenzoyl)-1,3-cyclohexanedione). J Inherit Metab Dis $21,507-517$.

Horlbeck, M.A., Gilbert, L.A., Villalta, J.E., Adamson, B., Pak, R.A., Chen, Y., Fields, A.P., Park, C.Y., Corn, J.E., Kampmann, M., et al. (2016). Compact and highly active next-generation libraries for CRISPR-mediated gene repression and activation. eLife 5, e19760.

Horlbeck, M.A., Liu, S.J., Chang, H.Y., Lim, D.A., and Weissman, J.S. (2020). Fitness effects of CRISPR/Cas9-targeting of long noncoding RNA genes. Nat Biotechnol 38, 573-576.

Hsu, P.D., Scott, D.A., Weinstein, J.A., Ran, F.A., Konermann, S., Agarwala, V., Li, Y., Fine, E.J., Wu, X., Shalem, O., et al. (2013). DNA targeting specificity of RNA-guided Cas9 nucleases. Nat Biotechnol 31, 827-832.

Hu, J.H., Miller, S.M., Geurts, M.H., Tang, W., Chen, L., Sun, N., Zeina, C. M., Gao, X., Rees, H.A., Lin, Z., et al. (2018). Evolved Cas9 variants with broad PAM compatibility and high DNA specificity. Nature 556, 57-63.

Hu, Z., Yu, L., Zhu, D., Ding, W., Wang, X., Zhang, C., Wang, L., Jiang, X., Shen, H., He, D., et al. (2014). Disruption of HPV16-E7 by CRISPR/Cas system induces apoptosis and growth inhibition in HPV16 positive human cervical cancer cells. Biomed Res Int 2014, 1-9.

Hu, Z., Ding, W., Zhu, D., Yu, L., Jiang, X., Wang, X., Zhang, C., Wang, L., Ji, T., Liu, D., et al. (2015). TALEN-mediated targeting of HPV oncogenes ameliorates HPV-related cervical malignancy. J Clin Invest $125,425-436$.

Huang, D., Qian, J., Shi, Z., Zhao, J., Fang, M., and Xu, Z. (2020a). CRISPR-Cas12a-assisted multicolor biosensor for semiquantitative point-of-use testing of the nopaline synthase terminator in genetically modified crops by unaided eyes. ACS Synth Biol 9, 3114-3123. 
Huang, M., Zhou, X., Wang, H., and Xing, D. (2018). Clustered regularly interspaced short palindromic repeats/Cas9 triggered isothermal amplification for site-specific nucleic acid detection. Anal Chem 90, 2193-2200.

Huang, W., Yu, L., Wen, D., Wei, D., Sun, Y., Zhao, H., Ye, Y., Chen, W., Zhu, Y., Wang, L., et al. (2020b). A CRISPR-Cas12a-based specific enhancer for more sensitive detection of SARS-CoV-2 infection. Ebiomedicine 61, 103036.

Huang, Y., Ding, C., Liang, P., Li, D., Tang, Y., Meng, W., Sun, H., Lu, H., Chen, Y., Chen, X., et al. (2019). HBB-deficient Macaca fascicularis monkey presents with human $\beta$-thalassemia. Protein Cell 10, 538-542.

Huang, Y., Paxton, W.A., Wolinsky, S.M., Neumann, A.U., Zhang, L., He, T., Kang, S., Ceradini, D., Jin, Z., Yazdanbakhsh, K., et al. (1996). The role of a mutant CCR5 allele in HIV-1 transmission and disease progression. Nat Med 2, 1240-1243.

Humbert, O., and Kiem, H.P. (2015). Long-term increase in fetal hemoglobin expression in nonhuman primates following transplantation of autologous Bcl11a nuclease-edited HSCs. Blood 126, 2035.

Humbert, O., Peterson, C.W., Norgaard, Z.K., Radtke, S., and Kiem, H.P. (2018). A nonhuman primate transplantation model to evaluate hematopoietic stem cell gene editing strategies for $\beta$ hemoglobinopathies. Mol Ther Methods Clin Dev 8, 75-86.

Ihry, R.J., Worringer, K.A., Salick, M.R., Frias, E., Ho, D., Theriault, K., Kommineni, S., Chen, J., Sondey, M., Ye, C., et al. (2018). p53 inhibits CRISPR-Cas9 engineering in human pluripotent stem cells. Nat Med 24, 939-946.

Ivics, Z., Li, M.A., Mátés, L., Boeke, J.D., Nagy, A., Bradley, A., and Izsvák, Z. (2009). Transposon-mediated genome manipulation in vertebrates. Nat Methods 6, 415-422.

Izpisua Belmonte, J.C., Callaway, E.M., Caddick, S.J., Churchland, P., Feng, G., Homanics, G.E., Lee, K.F., Leopold, D.A., Miller, C.T., Mitchell, J.F., et al. (2015). Brains, genes, and primates. Neuron 86, 617-631.

Jacobson, S.G., Cideciyan, A.V., Roman, A.J., Sumaroka, A., Schwartz, S. B., Heon, E., and Hauswirth, W.W. (2015). Improvement and decline in vision with gene therapy in childhood blindness. N Engl J Med 372, 1920-1926.

Jaitin, D.A., Weiner, A., Yofe, I., Lara-Astiaso, D., Keren-Shaul, H., David, E., Salame, T.M., Tanay, A., van Oudenaarden, A., and Amit, I. (2016). Dissecting immune circuits by linking CRISPR-pooled screens with single-cell RNA-seq. Cell 167, 1883-1896.e15.

Jasin, M., and Rothstein, R. (2013). Repair of strand breaks by homologous recombination. Cold Spring Harb Perspect Biol 5, a012740.

Jeong, Y.K., Song, B., and Bae, S. (2020). Current status and challenges of DNA base editing tools. Mol Ther 28, 1938-1952.

Ji, H., Jiang, Z., Lu, P., Ma, L., Li, C., Pan, H., Fu, Z., Qu, X., Wang, P., Deng, J., et al. (2016). Specific reactivation of latent HIV-1 by dCas9SunTag-VP64-mediated guide RNA targeting the HIV-1 promoter. Mol Ther 24, 508-521.

Jiang, C., Trudeau, S.J., Cheong, T.C., Guo, R., Teng, M., Wang, L.W., Wang, Z., Pighi, C., Gautier-Courteille, C., Ma, Y., et al. (2019). CRISPR/Cas9 screens reveal multiple layers of B cell CD40 regulation. Cell Rep 28, 1307-1322.e8.

Jiang, T., Zhang, X.O., Weng, Z., and Xue, W. (2021). Programming large target genomic deletion and concurrent insertion via a prime editingbased method: PEDAR. bioRxiv 2021.2005.2012.443800.

Jiao, C., Sharma, S., Dugar, G., Peeck, N.L., Bischler, T., Wimmer, F., Yu, Y., Barquist, L., Schoen, C., Kurzai, O., et al. (2021). Noncanonical crRNAs derived from host transcripts enable multiplexable RNA detection by Cas9. Science 372, 941-948.

Jin, S., Fei, H., Zhu, Z., Luo, Y., Liu, J., Gao, S., Zhang, F., Chen, Y.H., Wang, Y., and Gao, C. (2020). Rationally designed APOBEC3B cytosine base editors with improved specificity. Mol Cell 79, 728-740. e6.

Jin, S., Lin, Q., Luo, Y., Zhu, Z., Liu, G., Li, Y., Chen, K., Qiu, J.L., and Gao, C. (2021). Genome-wide specificity of prime editors in plants. Nat
Biotechnol 39, 1292-1299.

Jin, S., Zong, Y., Gao, Q., Zhu, Z., Wang, Y., Qin, P., Liang, C., Wang, D., Qiu, J.L., Zhang, F., et al. (2019). Cytosine, but not adenine, base editors induce genome-wide off-target mutations in rice. Science 364 , 292-295.

Jinek, M., Chylinski, K., Fonfara, I., Hauer, M., Doudna, J.A., and Charpentier, E. (2012). A programmable dual-RNA-guided DNA endonuclease in adaptive bacterial immunity. Science 337, 816-821.

Jinek, M., East, A., Cheng, A., Lin, S., Ma, E., and Doudna, J. (2013). RNA-programmed genome editing in human cells. eLife 2, e00471.

Joung, J., Engreitz, J.M., Konermann, S., Abudayyeh, O.O., Verdine, V.K., Aguet, F., Gootenberg, J.S., Sanjana, N.E., Wright, J.B., Fulco, C.P., et al. (2017). Genome-scale activation screen identifies a lncRNA locus regulating a gene neighbourhood. Nature 548, 343-346.

Joung, J., Ladha, A., Saito, M., Kim, N.G., Woolley, A.E., Segel, M., Barretto, R.P.J., Ranu, A., Macrae, R.K., Faure, G., et al. (2020). Detection of SARS-CoV-2 with SHERLOCK one-pot testing. N Engl J Med 383, 1492-1494.

Jung, I.Y., Kim, Y.Y., Yu, H.S., Lee, M., Kim, S., and Lee, J. (2018). CRISPR/Cas9-mediated knockout of DGK improves antitumor activities of human T cells. Cancer Res 78, 4692-4703.

Kaczmarek, J.C., Kowalski, P.S., and Anderson, D.G. (2017). Advances in the delivery of RNA therapeutics: from concept to clinical reality. Genome Med 9, 60.

Kang, Y., Chu, C., Wang, F., and Niu, Y. (2019). CRISPR/Cas9-mediated genome editing in nonhuman primates. Dis Model Mech 12.

Kennedy, E.M., and Cullen, B.R. (2017). Gene editing: A new tool for viral disease. Annu Rev Med 68, 401-411.

Khanna, K.K., Keating, K.E., Kozlov, S., Scott, S., Gatei, M., Hobson, K., Taya, Y., Gabrielli, B., Chan, D., Lees-Miller, S.P., et al. (1998). ATM associates with and phosphorylates p53: mapping the region of interaction. Nat Genet 20, 398-400.

Kim, D., Bae, S., Park, J., Kim, E., Kim, S., Yu, H.R., Hwang, J., Kim, J.I., and Kim, J.S. (2015). Digenome-seq: genome-wide profiling of CRISPR-Cas9 off-target effects in human cells. Nat Methods 12, 237-243.

Kim, D., Luk, K., Wolfe, S.A., and Kim, J.S. (2019). Evaluating and enhancing target specificity of gene-editing nucleases and deaminases. Annu Rev Biochem 88, 191-220.

Kim, D.Y., Moon, S.B., Ko, J.H., Kim, Y.S., and Kim, D. (2020). Unbiased investigation of specificities of prime editing systems in human cells. Nucleic Acids Res 48, 10576-10589.

Kim, S., Kim, D., Cho, S.W., Kim, J., and Kim, J.S. (2014). Highly efficient RNA-guided genome editing in human cells via delivery of purified Cas9 ribonucleoproteins. Genome Res 24, 1012-1019.

Kim, S., Koo, T., Jee, H.G., Cho, H.Y., Lee, G., Lim, D.G., Shin, H.S., and Kim, J.S. (2018). CRISPR RNAs trigger innate immune responses in human cells. Genome Res 28, 367-373.

Kim, Y.B., Komor, A.C., Levy, J.M., Packer, M.S., Zhao, K.T., and Liu, D. R. (2017). Increasing the genome-targeting scope and precision of base editing with engineered Cas9-cytidine deaminase fusions. Nat Biotechnol 35, 371-376.

Kim, Y.G., Cha, J., and Chandrasegaran, S. (1996). Hybrid restriction enzymes: zinc finger fusions to Fok I cleavage domain. Proc Natl Acad Sci USA 93, 1156-1160.

Klann, T.S., Black, J.B., Chellappan, M., Safi, A., Song, L., Hilton, I.B., Crawford, G.E., Reddy, T.E., and Gersbach, C.A. (2017). CRISPR-Cas9 epigenome editing enables high-throughput screening for functional regulatory elements in the human genome. Nat Biotechnol 35, 561-568.

Kleinstiver, B.P., Pattanayak, V., Prew, M.S., Tsai, S.Q., Nguyen, N.T., Zheng, Z., and Joung, J.K. (2016a). High-fidelity CRISPR-Cas9 nucleases with no detectable genome-wide off-target effects. Nature 529, 490-495.

Kleinstiver, B.P., Prew, M.S., Tsai, S.Q., Topkar, V.V., Nguyen, N.T., Zheng, Z., Gonzales, A.P.W., Li, Z., Peterson, R.T., Yeh, J.R.J., et al. (2015). Engineered CRISPR-Cas9 nucleases with altered PAM specificities. Nature 523, 481-485. 
Kleinstiver, B.P., Tsai, S.Q., Prew, M.S., Nguyen, N.T., Welch, M.M., Lopez, J.M., McCaw, Z.R., Aryee, M.J., and Joung, J.K. (2016b) Genome-wide specificities of CRISPR-Cas Cpf1 nucleases in human cells. Nat Biotechnol 34, 869-874.

Klompe, S.E., Vo, P.L.H., Halpin-Healy, T.S., and Sternberg, S.H. (2019). Transposon-encoded CRISPR-Cas systems direct RNA-guided DNA integration. Nature 571, 219-225.

Klug, A. (2010). The discovery of zinc fingers and their applications in gene regulation and genome manipulation. Annu Rev Biochem 79, 213-231.

Kocak, D.D., Josephs, E.A., Bhandarkar, V., Adkar, S.S., Kwon, J.B., and Gersbach, C.A. (2019). Increasing the specificity of CRISPR systems with engineered RNA secondary structures. Nat Biotechnol 37, 657666.

Komor, A.C., Kim, Y.B., Packer, M.S., Zuris, J.A., and Liu, D.R. (2016). Programmable editing of a target base in genomic DNA without doublestranded DNA cleavage. Nature 533, 420-424.

Komor, A.C., Zhao, K.T., Packer, M.S., Gaudelli, N.M., Waterbury, A.L., Koblan, L.W., Kim, Y.B., Badran, A.H., and Liu, D.R. (2017). Improved base excision repair inhibition and bacteriophage $\mathrm{Mu}$ Gam protein yields C:G-to-T:A base editors with higher efficiency and product purity. Sci Adv 3, eaao4774.

Konermann, S., Brigham, M.D., Trevino, A.E., Hsu, P.D., Heidenreich, M., Le Cong, M., Platt, R.J., Scott, D.A., Church, G.M., and Zhang, F. (2013). Optical control of mammalian endogenous transcription and epigenetic states. Nature 500, 472-476.

Konermann, S., Lotfy, P., Brideau, N.J., Oki, J., Shokhirev, M.N., and Hsu, P.D. (2018). Transcriptome engineering with RNA-targeting type VI-D CRISPR effectors. Cell 173, 665-676.e14.

Korkmaz, G., Lopes, R., Ugalde, A.P., Nevedomskaya, E., Han, R., Myacheva, K., Zwart, W., Elkon, R., and Agami, R. (2016). Functional genetic screens for enhancer elements in the human genome using CRISPR-Cas9. Nat Biotechnol 34, 192-198.

Korkmaz, G., Manber, Z., Lopes, R., Prekovic, S., Schuurman, K., Kim, Y., Teunissen, H., Flach, K., Wit, E., Galli, G.G., et al. (2019). A CRISPRCas9 screen identifies essential CTCF anchor sites for estrogen receptor-driven breast cancer cell proliferation. Nucleic Acids Res 47, 9557-9572.

Kosicki, M., Tomberg, K., and Bradley, A. (2018). Repair of double-strand breaks induced by CRISPR-Cas9 leads to large deletions and complex rearrangements. Nat Biotechnol 36, 765-771.

Kovač, A., Miskey, C., Menzel, M., Grueso, E., Gogol-Döring, A., and Ivics, Z. (2020). RNA-guided retargeting of Sleeping Beauty transposition in human cells. eLife 9, e53868.

Kunkel, T.A., and Erie, D.A. (2015). Eukaryotic mismatch repair in relation to DNA replication. Annu Rev Genet 49, 291-313.

Kurata, J.S., and Lin, R.J. (2018). MicroRNA-focused CRISPR-Cas9 library screen reveals fitness-associated miRNAs. RNA 24, 966-981.

Ladowski, J., Martens, G., Estrada, J., Tector, M., and Tector, J. (2019). The desirable donor pig to eliminate all xenoreactive antigens. Xenotransplantation 26, e12504.

Landrum, M.J., Lee, J.M., Riley, G.R., Jang, W., Rubinstein, W.S., Church, D.M., and Maglott, D.R. (2014). ClinVar: public archive of relationships among sequence variation and human phenotype. Nucl Acids Res 42, D980-D985.

Lansing, F., Mukhametzyanova, L., Rojo-Romanos, T., Iwasawa, K., Kimura, M., Paszkowski-Rogacz, M., Karpinski, J., Grass, T., Sonntag, J., Schneider, P.M., et al. (2020a). Correction of a Factor VIII genomic inversion with designer-recombinases. bioRxiv 2020.2011.2002.328013

Lansing, F., Paszkowski-Rogacz, M., Schmitt, L.T., Schneider, P.M., Rojo Romanos, T., Sonntag, J., and Buchholz, F. (2020b). A heterodimer of evolved designer-recombinases precisely excises a human genomic DNA locus. Nucleic Acids Res 48, 472-485.

Latella, M.C., Di Salvo, M.T., Cocchiarella, F., Benati, D., Grisendi, G., Comitato, A., Marigo, V., and Recchia, A. (2016). In vivo editing of the human mutant rhodopsin gene by electroporation of plasmid-based
CRISPR/Cas9 in the mouse retina. Mol Ther Nucleic Acids 5, e389.

Lee, J.K., Jeong, E., Lee, J., Jung, M., Shin, E., Kim, Y.H., Lee, K., Jung, I., Kim, D., Kim, S., et al. (2018). Directed evolution of CRISPR-Cas9 to increase its specificity. Nat Commun 9, 3048.

Lee, R.A., Puig, H.D., Nguyen, P.Q., Angenent-Mari, N.M., Donghia, N. M., McGee, J.P., Dvorin, J.D., Klapperich, C.M., Pollock, N.R., and Collins, J.J. (2020). Ultrasensitive CRISPR-based diagnostic for fieldapplicable detection of Plasmodium species in symptomatic and asymptomatic malaria. Proc Natl Acad Sci USA 117, 25722-25731.

Lei, L., Chen, H., Xue, W., Yang, B., Hu, B., Wei, J., Wang, L., Cui, Y., Li, W., Wang, J., et al. (2018). APOBEC3 induces mutations during repair of CRISPR-Cas9-generated DNA breaks. Nat Struct Mol Biol 25, 4552 .

Lei, Z., Meng, H., Lv, Z., Liu, M., Zhao, H., Wu, H., Zhang, X., Liu, L., Zhuang, Y., Yin, K., et al. (2021). Detect-seq reveals out-of-protospacer editing and target-strand editing by cytosine base editors. Nat Methods $18,643-651$.

Leung, R.K.K., Cheng, Q.X., Wu, Z.L., Khan, G., Liu, Y., Xia, H.Y., and Wang, J. (2021). CRISPR-Cas12-based nucleic acids detection systems. Methods doi: 10.1016/j.ymeth.2021.02.018.

Li, D., Qiu, Z., Shao, Y., Chen, Y., Guan, Y., Liu, M., Li, Y., Gao, N., Wang, L., Lu, X., et al. (2013). Heritable gene targeting in the mouse and rat using a CRISPR-Cas system. Nat Biotechnol 31, 681-683.

Li, H., Li, M., Yang, Y., Wang, F., Wang, F., and Li, C. (2021a). Aptamerlinked CRISPR/Cas12a-based immunoassay. Anal Chem 93, 32093216.

Li, H., Xing, S., Xu, J., He, Y., Lai, Y., Wang, Y., Zhang, G., Guo, S., Deng, M., Zeng, M., et al. (2021b). Aptamer-based CRISPR/Cas12a assay for the ultrasensitive detection of extracellular vesicle proteins. Talanta 221,121670

Li, J., Yang, S., Zuo, C., Dai, L., Guo, Y., and Xie, G. (2020a). Applying CRISPR-Cas12a as a signal amplifier to construct biosensors for NonDNA targets in ultralow concentrations. ACS Sens 5, 970-977.

Li, L., Li, S., Wu, N., Wu, J., Wang, G., Zhao, G., and Wang, J. (2019a). HOLMESv2: a CRISPR-Cas12b-assisted platform for nucleic acid detection and DNA methylation quantitation. ACS Synth Biol 8, 22282237.

Li, N., Tang, N., Cheng, C., Hu, T., Wei, X., Han, W., and Wang, H. (2020b). Improving the anti-solid tumor efficacy of CAR-T cells by inhibiting adenosine signaling pathway. Oncoimmunology 9, 1824643.

Li, P., Kleinstiver, B.P., Leon, M.Y., Prew, M.S., Navarro-Gomez, D., Greenwald, S.H., Pierce, E.A., Joung, J.K., and Liu, Q. (2018a). Allelespecific CRISPR-Cas9 genome editing of the single-base P23H mutation for rhodopsin-associated dominant retinitis pigmentosa. CRISPR J 1, 55-64.

Li, S., Li, X., Xue, W., Zhang, L., Yang, L.Z., Cao, S.M., Lei, Y.N., Liu, C. X., Guo, S.K., Shan, L., et al. (2021c). Screening for functional circular RNAs using the CRISPR-Cas13 system. Nat Methods 18, 51-59.

Li, S.Y., Cheng, Q.X., Liu, J.K., Nie, X.Q., Zhao, G.P., and Wang, J. (2018b). CRISPR-Cas12a has both cis-and trans-cleavage activities on single-stranded DNA. Cell Res 28, 491-493.

Li, S.Y., Cheng, Q.X., Wang, J.M., Li, X.Y., Zhang, Z.L., Gao, S., Cao, R. B., Zhao, G.P., and Wang, J. (2018c). CRISPR-Cas12a-assisted nucleic acid detection. Cell Discov 4, 20.

Li, X., Wang, Y., Liu, Y., Yang, B., Wang, X., Wei, J., Lu, Z., Zhang, Y., Wu, J., Huang, X., et al. (2018d). Base editing with a Cpfl-cytidine deaminase fusion. Nat Biotechnol 36, 324-327.

Li, Y., Li, S., Wang, J., and Liu, G. (2019b). CRISPR/Cas systems towards next-generation biosensing. Trends Biotechnol 37, 730-743.

Li, Y., Yao, L., Li, J., Chen, L., Song, Y., Cai, Z., and Yang, C. (2020c). Stability issues of RT-PCR testing of SARS-CoV-2 for hospitalized patients clinically diagnosed with COVID-19. J Med Virol 92, 903-908.

Li, Z., Zheng, W., Wang, H., Cheng, Y., Fang, Y., Wu, F., Sun, G., Sun, G., Lv, C., and Hui, B. (2021d). Application of animal models in cancer research: recent progress and future prospects. Cancer Manag Res Volume 13, 2455-2475.

Liang, M., Li, Z., Wang, W., Liu, J., Liu, L., Zhu, G., Karthik, L., Wang, 
M., Wang, K.F., Wang, Z., et al. (2019). A CRISPR-Cas12a-derived biosensing platform for the highly sensitive detection of diverse small molecules. Nat Commun 10, 3672.

Liang, Y., Zhang, G., Li, Q., Han, L., Hu, X., Guo, Y., Tao, W., Zhao, X., Guo, M., Gan, T., et al. (2021). TRIM26 is a critical host factor for HCV replication and contributes to host tropism. Sci Adv 7, eabd9732.

Lin, Q., Jin, S., Zong, Y., Yu, H., Zhu, Z., Liu, G., Kou, L., Wang, Y., Qiu, J.L., Li, J., et al. (2021). High-efficiency prime editing with optimized, paired pegRNAs in plants. Nat Biotechnol 39, 923-927.

Lin, Q., Lv, J.N., Wu, K.C., Zhang, C.J., Liu, Q., and Jin, Z.B. (2020). Generation of nonhuman primate model of cone dysfunction through in situ AAV-mediated CNGB3 ablation. Mol Ther Methods Clin Dev 18, 869-879.

Ling, S., Yang, S., Hu, X., Yin, D., Dai, Y., Qian, X., Wang, D., Pan, X., Hong, J., Sun, X., et al. (2021). Lentiviral delivery of co-packaged Cas9 mRNA and a Vegfa-targeting guide RNA prevents wet age-related macular degeneration in mice. Nat Biomed Eng 5, 144-156.

Liu, P.F., Zhao, K.R., Liu, Z.J., Wang, L., Ye, S.Y., and Liang, G.X. (2021a). Cas12a-based electrochemiluminescence biosensor for target amplification-free DNA detection. Biosens Bioelectron 176, 112954.

Liu, R., Paxton, W.A., Choe, S., Ceradini, D., Martin, S.R., Horuk, R., MacDonald, M.E., Stuhlmann, H., Koup, R.A., and Landau, N.R. (1996). Homozygous defect in HIV-1 coreceptor accounts for resistance of some multiply-exposed individuals to HIV-1 infection. Cell 86, 367377.

Liu, S.J., Horlbeck, M.A., Cho, S.W., Birk, H.S., Malatesta, M., He, D., Attenello, F.J., Villalta, J.E., Cho, M.Y., Chen, Y., et al. (2017a). CRISPRi-based genome-scale identification of functional long noncoding RNA loci in human cells. Science 355.

Liu, X., Zhang, Y., Cheng, C., Cheng, A.W., Zhang, X., Li, N., Xia, C., Wei, X., Liu, X., and Wang, H. (2017b). CRISPR-Cas9-mediated multiplex gene editing in CAR-T cells. Cell Res 27, 154-157.

Liu, X.S., Wu, H., Ji, X., Stelzer, Y., Wu, X., Czauderna, S., Shu, J., Dadon, D., Young, R.A., and Jaenisch, R. (2016). Editing DNA methylation in the mammalian genome. Cell 167, 233-247.e17.

Liu, Y., Cao, Z., Wang, Y., Guo, Y., Xu, P., Yuan, P., Liu, Z., He, Y., and Wei, W. (2018a). Genome-wide screening for functional long noncoding RNAs in human cells by Cas 9 targeting of splice sites. Nat Biotechnol 36, 1203-1210.

Liu, Y., Liu, Z., Cao, Z., and Wei, W. (2020). Reply to: fitness effects of CRISPR/Cas9-targeting of long noncoding RNA genes. Nat Biotechnol 38, 577-578.

Liu, Y., Yang, G., Huang, S., Li, X., Wang, X., Li, G., Chi, T., Chen, Y., Huang, X., and Wang, X. (2021b). Enhancing prime editing by Csy4mediated processing of pegRNA. Cell Res 31, 1134-1136.

Liu, Z., Cai, Y., Wang, Y., Nie, Y., Zhang, C., Xu, Y., Zhang, X., Lu, Y., Wang, Z., Poo, M., et al. (2018b). Cloning of macaque monkeys by somatic cell nuclear transfer. Cell 172, 881-887.e7.

Lu, S., Li, F., Chen, Q., Wu, J., Duan, J., Lei, X., Zhang, Y., Zhao, D., Bu, Z., and Yin, H. (2020). Rapid detection of African swine fever virus using Cas12a-based portable paper diagnostics. Cell Discov 6, 18.

Luo, W., Galvan, D.L., Woodard, L.E., Dorset, D., Levy, S., and Wilson, M. H. (2017). Comparative analysis of chimeric ZFP-, TALE- and Cas9piggyBac transposases for integration into a single locus in human cells. Nucleic Acids Res 45, 8411-8422.

Ma, Y., Zhang, J., Yin, W., Zhang, Z., Song, Y., and Chang, X. (2016). Targeted AID-mediated mutagenesis (TAM) enables efficient genomic diversification in mammalian cells. Nat Methods 13, 1029-1035.

Ma, Y., Zhang, X., Shen, B., Lu, Y., Chen, W., Ma, J., Bai, L., Huang, X., and Zhang, L. (2014). Generating rats with conditional alleles using CRISPR/Cas9. Cell Res 24, 122-125.

Maeder, M.L., Stefanidakis, M., Wilson, C.J., Baral, R., Barrera, L.A., Bounoutas, G.S., Bumcrot, D., Chao, H., Ciulla, D.M., DaSilva, J.A., et al. (2019). Development of a gene-editing approach to restore vision loss in Leber congenital amaurosis type 10. Nat Med 25, 229-233.

Maguire, A.M., High, K.A., Auricchio, A., Wright, J.F., Pierce, E.A., Testa, F., Mingozzi, F., Bennicelli, J.L., Ying, G., Rossi, S., et al. (2009). Age- dependent effects of RPE65 gene therapy for Leber's congenital amaurosis: a phase 1 dose-escalation trial. Lancet 374, 1597-1605.

Maguire, A.M., Simonelli, F., Pierce, E.A., Pugh Jr, E.N., Mingozzi, F., Bennicelli, J., Banfi, S., Marshall, K.A., Testa, F., Surace, E.M., et al. (2008). Safety and efficacy of gene transfer for Leber's congenital amaurosis. N Engl J Med 358, 2240-2248.

Makarova, K.S., Wolf, Y.I., Iranzo, J., Shmakov, S.A., Alkhnbashi, O.S., Brouns, S.J.J., Charpentier, E., Cheng, D., Haft, D.H., Horvath, P., et al. (2020). Evolutionary classification of CRISPR-Cas systems: a burst of class 2 and derived variants. Nat Rev Microbiol 18, 67-83.

Mali, P., Yang, L., Esvelt, K.M., Aach, J., Guell, M., DiCarlo, J.E., Norville, J.E., and Church, G.M. (2013). RNA-guided human genome engineering via Cas9. Science 339, 823-826.

Mandal, P.K., Ferreira, L.M.R., Collins, R., Meissner, T.B., Boutwell, C.L., Friesen, M., Vrbanac, V., Garrison, B.S., Stortchevoi, A., Bryder, D., et al. (2014). Efficient ablation of genes in human hematopoietic stem and effector cells using CRISPR/Cas9. Cell Stem Cell 15, 643-652.

Mao, X.Y., Dai, J.X., Zhou, H.H., Liu, Z.Q., and Jin, W.L. (2016). Brain tumor modeling using the CRISPR/Cas9 system: state of the art and view to the future. Oncotarget 7, 33461-33471.

Maresch, R., Mueller, S., Veltkamp, C., Öllinger, R., Friedrich, M., Heid, I., Steiger, K., Weber, J., Engleitner, T., Barenboim, M., et al. (2016). Multiplexed pancreatic genome engineering and cancer induction by transfection-based CRISPR/Cas9 delivery in mice. Nat Commun 7, 10770 .

Martin, R.M., Ikeda, K., Cromer, M.K., Uchida, N., Nishimura, T., Romano, R., Tong, A.J., Lemgart, V.T., Camarena, J., Pavel-Dinu, M., et al. (2019). Highly efficient and marker-free genome editing of human pluripotent stem cells by CRISPR-Cas9 RNP and AAV6 donor-mediated homologous recombination. Cell Stem Cell 24, 821828.e5.

Martinez-Lage, M., Torres-Ruiz, R., and Rodriguez-Perales, S. (2017). CRISPR/Cas9 technology: applications and human disease modeling. In: Progress in Molecular Biology and Translational Science. New York: Academic Press. 23-48.

Meinke, G., Bohm, A., Hauber, J., Pisabarro, M.T., and Buchholz, F. (2016). Cre recombinase and other tyrosine recombinases. Chem Rev $116,12785-12820$.

Menchaca, A., Dos Santos-Neto, P.C., Souza-Neves, M., Cuadro, F., Mulet, A.P., Tesson, L., Chenouard, V., Guiffès, A., Heslan, J.M., Gantier, M., et al. (2020). Otoferlin gene editing in sheep via CRISPR-assisted ssODN-mediated Homology Directed Repair. Sci Rep 10, 5995.

Merkle, T., Merz, S., Reautschnig, P., Blaha, A., Li, Q., Vogel, P., Wettengel, J., Li, J.B., and Stafforst, T. (2019). Precise RNA editing by recruiting endogenous ADARs with antisense oligonucleotides. Nat Biotechnol 37, 133-138.

Miller, J., McLachlan, A.D., and Klug, A. (1985). Repetitive zinc-binding domains in the protein transcription factor IIIA from Xenopus oocytes. EMBO J 4, 1609-1614.

Miller, J.C., Tan, S., Qiao, G., Barlow, K.A., Wang, J., Xia, D.F., Meng, X., Paschon, D.E., Leung, E., Hinkley, S.J., et al. (2011). A TALE nuclease architecture for efficient genome editing. Nat Biotechnol 29, 143-148.

Miller, S.M., Wang, T., Randolph, P.B., Arbab, M., Shen, M.W., Huang, T. P., Matuszek, Z., Newby, G.A., Rees, H.A., and Liu, D.R. (2020). Continuous evolution of SpCas9 variants compatible with non-G PAMs. Nat Biotechnol 38, 471-481.

Mimitou, E.P., Cheng, A., Montalbano, A., Hao, S., Stoeckius, M., Legut, M., Roush, T., Herrera, A., Papalexi, E., Ouyang, Z., et al. (2019). Multiplexed detection of proteins, transcriptomes, clonotypes and CRISPR perturbations in single cells. Nat Methods 16, 409-412.

Mojica, F.J.M., Díez-Villaseñor, C., García-Martínez, J., and Soria, E. (2005). Intervening sequences of regularly spaced prokaryotic repeats derive from foreign genetic elements. J Mol Evol 60, 174-182.

Moreno, A.M., Palmer, N., Alemán, F., Chen, G., Pla, A., Jiang, N., Leong Chew, W., Law, M., and Mali, P. (2019). Immune-orthogonal orthologues of AAV capsids and of Cas9 circumvent the immune response to the administration of gene therapy. Nat Biomed Eng 3, 806- 
816

Moscou, M.J., and Bogdanove, A.J. (2009). A simple cipher governs DNA recognition by TAL effectors. Science 326, 1501.

Mu, W., Tang, N., Cheng, C., Sun, W., Wei, X., and Wang, H. (2019). In vitro transcribed sgRNA causes cell death by inducing interferon release. Protein Cell 10, 461-465.

Mukama, O., Wu, J., Li, Z., Liang, Q., Yi, Z., Lu, X., Liu, Y., Liu, Y., Hussain, M., Makafe, G.G., et al. (2020). An ultrasensitive and specific point-of-care CRISPR/Cas 12 based lateral flow biosensor for the rapid detection of nucleic acids. Biosens Bioelectron 159, 112143.

Müller, U., and Barr-Gillespie, P.G. (2015). New treatment options for hearing loss. Nat Rev Drug Discov 14, 346-365.

Mussolino, C., Morbitzer, R., Lütge, F., Dannemann, N., Lahaye, T., and Cathomen, T. (2011). A novel TALE nuclease scaffold enables high genome editing activity in combination with low toxicity. Nucleic Acids Res 39, 9283-9293.

Mussolino, C., Alzubi, J., Fine, E.J., Morbitzer, R., Cradick, T.J., Lahaye, T., Bao, G., and Cathomen, T. (2014). TALENs facilitate targeted genome editing in human cells with high specificity and low cytotoxicity. Nucleic Acids Res 42, 6762-6773.

Musunuru, K., Chadwick, A.C., Mizoguchi, T., Garcia, S.P., DeNizio, J.E., Reiss, C.W., Wang, K., Iyer, S., Dutta, C., Clendaniel, V., et al. (2021). In vivo CRISPR base editing of PCSK9 durably lowers cholesterol in primates. Nature 593, 429-434.

Myhrvold, C., Freije, C.A., Gootenberg, J.S., Abudayyeh, O.O., Metsky, H. C., Durbin, A.F., Kellner, M.J., Tan, A.L., Paul, L.M., Parham, L.A., et al. (2018). Field-deployable viral diagnostics using CRISPR-Cas13. Science $360,444-448$.

Najm, F.J., Strand, C., Donovan, K.F., Hegde, M., Sanson, K.R., Vaimberg, E.W., Sullender, M.E., Hartenian, E., Kalani, Z., Fusi, N., et al. (2018) Orthologous CRISPR-Cas9 enzymes for combinatorial genetic screens. Nat Biotechnol 36, 179-189.

Nakamura, M., Gao, Y., Dominguez, A.A., and Qi, L.S. (2021). CRISPR technologies for precise epigenome editing. Nat Cell Biol 23, 11-22.

Naldini, L. (2011). Ex vivo gene transfer and correction for cell-based therapies. Nat Rev Genet 12, 301-315.

Newby, G.A., Yen, J.S., Woodard, K.J., Mayuranathan, T., Lazzarotto, C. R., Li, Y., Sheppard-Tillman, H., Porter, S.N., Yao, Y., Mayberry, K., et al. (2021). Base editing of haematopoietic stem cells rescues sickle cell disease in mice. Nature 595, 295-302.

Nguyen, G.N., Everett, J.K., Kafle, S., Roche, A.M., Raymond, H.E., Leiby, J., Wood, C., Assenmacher, C.A., Merricks, E.P., Long, C.T., et al. (2021). A long-term study of AAV gene therapy in dogs with hemophilia A identifies clonal expansions of transduced liver cells. Nat Biotechnol 39, 47-55.

Nguyen, L.T., Smith, B.M., and Jain, P.K. (2020). Enhancement of transcleavage activity of Cas12a with engineered crRNA enables amplified nucleic acid detection. Nat Commun 11, 4906.

Ning, B., Yu, T., Zhang, S., Huang, Z., Tian, D., Lin, Z., Niu, A., Golden, N., Hensley, K., Threeton, B., et al. (2021). A smartphone-read ultrasensitive and quantitative saliva test for COVID-19. Sci Adv 7, eabe 3703

Nishida, K., Arazoe, T., Yachie, N., Banno, S., Kakimoto, M., Tabata, M., Mochizuki, M., Miyabe, A., Araki, M., Hara, K.Y., et al. (2016). Targeted nucleotide editing using hybrid prokaryotic and vertebrate adaptive immune systems. Science 353.

Nishimasu, H., Ran, F.A., Hsu, P.D., Konermann, S., Shehata, S.I., Dohmae, N., Ishitani, R., Zhang, F., and Nureki, O. (2014). Crystal structure of Cas9 in complex with guide RNA and target DNA. Cell 156, 935-949.

Nishimasu, H., Shi, X., Ishiguro, S., Gao, L., Hirano, S., Okazaki, S., Noda, T., Abudayyeh, O.O., Gootenberg, J.S., Mori, H., et al. (2018). Engineered CRISPR-Cas9 nuclease with expanded targeting space. Science 361, 1259-1262.

Niu, D., Wei, H.J., Lin, L., George, H., Wang, T., Lee, I.H., Zhao, H.Y., Wang, Y., Kan, Y., Shrock, E., et al. (2017). Inactivation of porcine endogenous retrovirus in pigs using CRISPR-Cas9. Science 357, 1303-
1307.

Niu, Y., Shen, B., Cui, Y., Chen, Y., Wang, J., Wang, L., Kang, Y., Zhao, X., $\mathrm{Si}$, W., Li, W., et al. (2014). Generation of gene-modified cynomolgus monkey via Cas9/RNA-mediated gene targeting in one-cell embryos. Cell 156, 836-843.

Norman, T.M., Horlbeck, M.A., Replogle, J.M., Ge, A.Y., Xu, A., Jost, M., Gilbert, L.A., and Weissman, J.S. (2019). Exploring genetic interaction manifolds constructed from rich single-cell phenotypes. Science 365 , 786-793.

Nouri, R., Jiang, Y., Lian, X.L., and Guan, W. (2020). Sequence-specific recognition of HIV-1 DNA with solid-state CRISPR-Cas12a-assisted nanopores (SCAN). ACS Sens 5, 1273-1280.

Novarino, G. (2017). Rett syndrome modeling goes simian. Sci Transl Med 9.

Nuñez, J.K., Chen, J., Pommier, G.C., Cogan, J.Z., Replogle, J.M., Adriaens, C., Ramadoss, G.N., Shi, Q., Hung, K.L., Samelson, A.J., et al. (2021). Genome-wide programmable transcriptional memory by CRISPR-based epigenome editing. Cell 184, 2503-2519.e17.

Owens, J.B., Urschitz, J., Stoytchev, I., Dang, N.C., Stoytcheva, Z., Belcaid, M., Maragathavally, K.J., Coates, C.J., Segal, D.J., and Moisyadi, S. (2012). Chimeric piggyBac transposases for genomic targeting in human cells. Nucleic Acids Res 40, 6978-6991.

Owens, J.B., Mauro, D., Stoytchev, I., Bhakta, M.S., Kim, M.S., Segal, D. J., and Moisyadi, S. (2013). Transcription activator like effector (TALE)-directed piggyBac transposition in human cells. Nucleic Acids Res 41, 9197-9207.

Pan, B., Géléoc, G.S., Asai, Y., Horwitz, G.C., Kurima, K., Ishikawa, K., Kawashima, Y., Griffith, A.J., and Holt, J.R. (2013). TMC1 and TMC2 are components of the mechanotransduction channel in hair cells of the mammalian inner ear. Neuron 79, 504-515.

Pankowicz, F.P., Barzi, M., Legras, X., Hubert, L., Mi, T., Tomolonis, J.A., Ravishankar, M., Sun, Q., Yang, D., Borowiak, M., et al. (2016). Reprogramming metabolic pathways in vivo with CRISPR/Cas9 genome editing to treat hereditary tyrosinaemia. Nat Commun 7, 12642.

Pardee, K., Green, A.A., Takahashi, M.K., Braff, D., Lambert, G., Lee, J. W., Ferrante, T., Ma, D., Donghia, N., Fan, M., et al. (2016). Rapid, low-cost detection of Zika virus using programmable biomolecular components. Cell 165, 1255-1266.

Patchsung, M., Jantarug, K., Pattama, A., Aphicho, K., Suraritdechachai, S., Meesawat, P., Sappakhaw, K., Leelahakorn, N., Ruenkam, T., Wongsatit, T., et al. (2020). Clinical validation of a Cas13-based assay for the detection of SARS-CoV-2 RNA. Nat Biomed Eng 4, 1140-1149.

Pattanayak, V., Ramirez, C.L., Joung, J.K., and Liu, D.R. (2011). Revealing off-target cleavage specificities of zinc-finger nucleases by in vitro selection. Nat Methods 8, 765-770.

Peng, J., Zhou, Y., Zhu, S., and Wei, W. (2015). High-throughput screens in mammalian cells using the CRISPR-Cas9 system. FEBS J 282, 2089 2096.

Perez, E.E., Wang, J., Miller, J.C., Jouvenot, Y., Kim, K.A., Liu, O., Wang, N., Lee, G., Bartsevich, V.V., Lee, Y.L., et al. (2008). Establishment of HIV-1 resistance in $\mathrm{CD}^{+} \mathrm{T}$ cells by genome editing using zinc-finger nucleases. Nat Biotechnol 26, 808-816.

Pickar-Oliver, A., and Gersbach, C.A. (2019). The next generation of CRISPR-Cas technologies and applications. Nat Rev Mol Cell Biol 20, 490-507.

Platt, R.J., Chen, S., Zhou, Y., Yim, M.J., Swiech, L., Kempton, H.R., Dahlman, J.E., Parnas, O., Eisenhaure, T.M., Jovanovic, M., et al. (2014). CRISPR-Cas9 knockin mice for genome editing and cancer modeling. Cell 159, 440-455.

Pourcel, C., Salvignol, G., and Vergnaud, G. (2005). CRISPR elements in Yersinia pestis acquire new repeats by preferential uptake of bacteriophage DNA, and provide additional tools for evolutionary studies. Microbiology 151, 653-663.

Provasi, E., Genovese, P., Lombardo, A., Magnani, Z., Liu, P.Q., Reik, A., Chu, V., Paschon, D.E., Zhang, L., Kuball, J., et al. (2012). Editing T cell specificity towards leukemia by zinc finger nucleases and lentiviral gene transfer. Nat Med 18, 807-815. 
Qi, L.S., Larson, M.H., Gilbert, L.A., Doudna, J.A., Weissman, J.S., Arkin, A.P., and Lim, W.A. (2013). Repurposing CRISPR as an RNA-guided platform for sequence-specific control of gene expression. Cell 152, $1173-1183$.

Qiu, X.Y., Zhu, L.Y., Zhu, C.S., Ma, J.X., Hou, T., Wu, X.M., Xie, S.S., Min, L., Tan, D.A., Zhang, D.Y., et al. (2018). Highly effective and lowcost microRNA detection with CRISPR-Cas9. ACS Synth Biol 7, 807813.

Qu, L., Yi, Z., Zhu, S., Wang, C., Cao, Z., Zhou, Z., Yuan, P., Yu, Y., Tian, F., Liu, Z., et al. (2019). Programmable RNA editing by recruiting endogenous ADAR using engineered RNAs. Nat Biotechnol 37, 10591069.

Rajagopal, N., Srinivasan, S., Kooshesh, K., Guo, Y., Edwards, M.D., Banerjee, B., Syed, T., Emons, B.J.M., Gifford, D.K., and Sherwood, R. I. (2016). High-throughput mapping of regulatory DNA. Nat Biotechnol 34, 167-174.

Ramakrishna, S., Kwaku Dad, A.B., Beloor, J., Gopalappa, R., Lee, S.K., and Kim, H. (2014). Gene disruption by cell-penetrating peptidemediated delivery of Cas9 protein and guide RNA. Genome Res 24, $1020-1027$.

Ramanan, V., Shlomai, A., Cox, D.B.T., Schwartz, R.E., Michailidis, E., Bhatta, A., Scott, D.A., Zhang, F., Rice, C.M., and Bhatia, S.N. (2015). CRISPR/Cas9 cleavage of viral DNA efficiently suppresses hepatitis B virus. Sci Rep 5, 10833.

Ran, F.A., Cong, L., Yan, W.X., Scott, D.A., Gootenberg, J.S., Kriz, A.J., Zetsche, B., Shalem, O., Wu, X., Makarova, K.S., et al. (2015). In vivo genome editing using Staphylococcus aureus Cas9. Nature 520, 186191.

Ran, F.A., Hsu, P.D., Lin, C.Y., Gootenberg, J.S., Konermann, S., Trevino, A.E., Scott, D.A., Inoue, A., Matoba, S., Zhang, Y., et al. (2013). Double nicking by RNA-guided CRISPR Cas9 for enhanced genome editing specificity. Cell 154, 1380-1389.

Raper, S.E., Chirmule, N., Lee, F.S., Wivel, N.A., Bagg, A., Gao, G., Wilson, J.M., and Batshaw, M.L. (2003). Fatal systemic inflammatory response syndrome in a ornithine transcarbamylase deficient patient following adenoviral gene transfer. Mol Genet Metab 80, 148-158.

Rasys, A.M., Park, S., Ball, R.E., Alcala, A.J., Lauderdale, J.D., and Menke, D.B. (2019). CRISPR-Cas9 gene editing in lizards through microinjection of unfertilized oocytes. Cell Rep 28, 2288-2292.e3.

Rees, H.A., Komor, A.C., Yeh, W.H., Caetano-Lopes, J., Warman, M., Edge, A.S.B., and Liu, D.R. (2017). Improving the DNA specificity and applicability of base editing through protein engineering and protein delivery. Nat Commun 8, 15790.

Reik, A., Zhou, Y., Wagner, J., Hamlett, A., Mendel, M., Liu, P.Q., Lee, G., Paschon, D., Rebar, E., and Ando, D. (2008). Zinc finger nucleases targeting the glucocorticoid receptor allow IL-13 zetakine transgenic CTLs to kill glioblastoma cells in vivo in the presence of immunosuppressing glucocorticoids. In: AACR Annual Meeting. San Diego, CA.

Ren, J., Liu, X., Fang, C., Jiang, S., June, C.H., and Zhao, Y. (2017). Multiplex genome editing to generate universal CAR T cells resistant to PD1 inhibition. Clin Cancer Res 23, 2255-2266.

Ren, Q., Li, C., Yuan, P., Cai, C., Zhang, L., Luo, G.G., and Wei, W. (2015). A Dual-reporter system for real-time monitoring and high-throughput CRISPR/Cas9 library screening of the hepatitis C virus. Sci Rep 5, 8865.

Replogle, J.M., Norman, T.M., Xu, A., Hussmann, J.A., Chen, J., Cogan, J. Z., Meer, E.J., Terry, J.M., Riordan, D.P., Srinivas, N., et al. (2020). Combinatorial single-cell CRISPR screens by direct guide RNA capture and targeted sequencing. Nat Biotechnol 38, 954-961.

Richter, M.F., Zhao, K.T., Eton, E., Lapinaite, A., Newby, G.A., Thuronyi, B.W., Wilson, C., Koblan, L.W., Zeng, J., Bauer, D.E., et al. (2020). Phage-assisted evolution of an adenine base editor with improved Cas domain compatibility and activity. Nat Biotechnol 38, 883-891.

Rodriguez, C.M., and Todd, P.K. (2019). New pathologic mechanisms in nucleotide repeat expansion disorders. Neurobiol Dis 130, 104515.

Rothgangl, T., Dennis, M.K., Lin, P.J.C., Oka, R., Witzigmann, D., Villiger,
L., Qi, W., Hruzova, M., Kissling, L., Lenggenhager, D., et al. (2021). In vivo adenine base editing of PCSK9 in macaques reduces LDL cholesterol levels. Nat Biotechnol 39, 949-957.

Rouet, P., Smih, F., and Jasin, M. (1994). Introduction of double-strand breaks into the genome of mouse cells by expression of a rare-cutting endonuclease. Mol Cell Biol 14, 8096-8106.

Ruan, G.X., Barry, E., Yu, D., Lukason, M., Cheng, S.H., and Scaria, A. (2017). CRISPR/Cas9-mediated genome editing as a therapeutic approach for leber congenital amaurosis 10. Mol Ther 25, 331-341.

Rubin, A.J., Parker, K.R., Satpathy, A.T., Qi, Y., Wu, B., Ong, A.J., Mumbach, M.R., Ji, A.L., Kim, D.S., Cho, S.W., et al. (2019). Coupled single-cell CRISPR screening and epigenomic profiling reveals causal gene regulatory networks. Cell 176, 361-376.e17.

Rudich, P., and Lamitina, T. (2018). Models and mechanisms of repeat expansion disorders: a worm's eye view. J Genet 97, 665-677.

Rupp, L.J., Schumann, K., Roybal, K.T., Gate, R.E., Ye, C.J., Lim, W.A., and Marson, A. (2017). CRISPR/Cas9-mediated PD-1 disruption enhances anti-tumor efficacy of human chimeric antigen receptor $\mathrm{T}$ cells. Sci Rep 7, 737.

Russell, S., Bennett, J., Wellman, J.A., Chung, D.C., Yu, Z.F., Tillman, A., Wittes, J., Pappas, J., Elci, O., McCague, S., et al. (2017). Efficacy and safety of voretigene neparvovec (AAV2-hRPE65v2) in patients with RPE65 -mediated inherited retinal dystrophy: a randomised, controlled, open-label, phase 3 trial. Lancet 390, 849-860.

Ryczek, N., Hryhorowicz, M., Zeyland, J., Lipiński, D., and Słomski, R. (2021). CRISPR/Cas technology in pig-to-human xenotransplantation research. Int J Mol Sci 22, 3196.

Ryu, S.M., Koo, T., Kim, K., Lim, K., Baek, G., Kim, S.T., Kim, H.S., Kim, D.E., Lee, H., Chung, E., et al. (2018). Adenine base editing in mouse embryos and an adult mouse model of Duchenne muscular dystrophy. Nat Biotechnol 36, 536-539.

Saayman, S.M., Lazar, D.C., Scott, T.A., Hart, J.R., Takahashi, M., Burnett, J.C., Planelles, V., Morris, K.V., and Weinberg, M.S. (2016). Potent and targeted activation of latent HIV-1 using the CRISPR/dCas9 activator complex. Mol Ther 24, 488-498.

Salter, J.D., Bennett, R.P., and Smith, H.C. (2016). The APOBEC Protein family: united by structure, divergent in function. Trends Biochem Sci 41, 578-594.

Samaridou, E., Heyes, J., and Lutwyche, P. (2020). Lipid nanoparticles for nucleic acid delivery: current perspectives. Adv Drug Deliv Rev 154$155,37-63$.

Sánchez-Rivera, F.J., Papagiannakopoulos, T., Romero, R., Tammela, T., Bauer, M.R., Bhutkar, A., Joshi, N.S., Subbaraj, L., Bronson, R.T., Xue, W., et al. (2014). Rapid modelling of cooperating genetic events in cancer through somatic genome editing. Nature 516, 428-431.

Sato, K., Oiwa, R., Kumita, W., Henry, R., Sakuma, T., Ito, R., Nozu, R., Inoue, T., Katano, I., Sato, K., et al. (2016). Generation of a nonhuman primate model of severe combined immunodeficiency using highly efficient genome editing. Cell Stem Cell 19, 127-138.

Savoji, H., Mohammadi, M.H., Rafatian, N., Toroghi, M.K., Wang, E.Y., Zhao, Y., Korolj, A., Ahadian, S., and Radisic, M. (2019). Cardiovascular disease models: A game changing paradigm in drug discovery and screening. Biomaterials 198, 3-26.

Schmid-Burgk, J.L., Gao, L., Li, D., Gardner, Z., Strecker, J., Lash, B., and Zhang, F. (2020). Highly parallel profiling of Cas9 variant specificity. Mol Cell 78, 794-800.e8.

Seidah, N.G., Awan, Z., Chrétien, M., and Mbikay, M. (2014). PCSK9: a key modulator of cardiovascular health. Circ Res 114, 1022-1036.

Shalem, O., Sanjana, N.E., Hartenian, E., Shi, X., Scott, D.A., Mikkelsen, T.S., Heckl, D., Ebert, B.L., Root, D.E., Doench, J.G., et al. (2014). Genome-scale CRISPR-Cas9 knockout screening in human cells. Science 343, 84-87.

Shalem, O., Sanjana, N.E., and Zhang, F. (2015). High-throughput functional genomics using CRISPR-Cas9. Nat Rev Genet 16, 299-311.

Shams, A., Higgins, S.A., Fellmann, C., Laughlin, T.G., Oakes, B.L., Lew, R., Kim, S., Lukarska, M., Arnold, M., Staahl, B.T., et al. (2021). Comprehensive deletion landscape of CRISPR-Cas9 identifies minimal 
RNA-guided DNA-binding modules. Nat Commun 12, 5664.

Shao, N., Han, X., Song, Y., Zhang, P., and Qin, L. (2019). CRISPRCas12a coupled with platinum nanoreporter for visual quantification of SNVs on a volumetric bar-chart chip. Anal Chem 91, 12384-12391.

Shao, Y., Guan, Y., Wang, L., Qiu, Z., Liu, M., Chen, Y., Wu, L., Li, Y., Ma, X., Liu, M., et al. (2014). CRISPR/Cas-mediated genome editing in the rat via direct injection of one-cell embryos. Nat Protoc 9, 2493-2512.

Sharon, E., Chen, S.A.A., Khosla, N.M., Smith, J.D., Pritchard, J.K., and Fraser, H.B. (2018). Functional genetic variants revealed by massively parallel precise genome editing. Cell 175, 544-557.e16.

Shen, B., Zhang, W., Zhang, J., Zhou, J., Wang, J., Chen, L., Wang, L., Hodgkins, A., Iyer, V., Huang, X., et al. (2014). Efficient genome modification by CRISPR-Cas9 nickase with minimal off-target effects. Nat Methods 11, 399-402.

Shen, J.P., Zhao, D., Sasik, R., Luebeck, J., Birmingham, A., BojorquezGomez, A., Licon, K., Klepper, K., Pekin, D., Beckett, A.N., et al. (2017). Combinatorial CRISPR-Cas9 screens for de novo mapping of genetic interactions. Nat Methods 14, 573-576.

Shi, K., Xie, S., Tian, R., Wang, S., Lu, Q., Gao, D., Lei, C., Zhu, H., and Nie, Z. (2021). A CRISPR-Cas autocatalysis-driven feedback amplification network for supersensitive DNA diagnostics. Sci Adv 7, eabc7802.

Shiloh, Y., and Ziv, Y. (2013). The ATM protein kinase: regulating the cellular response to genotoxic stress, and more. Nat Rev Mol Cell Biol $14,197-210$.

Shinoda, H., Taguchi, Y., Nakagawa, R., Makino, A., Okazaki, S., Nakano, M., Muramoto, Y., Takahashi, C., Takahashi, I., Ando, J., et al. (2021). Amplification-free RNA detection with CRISPR-Cas13. Commun Biol 4, 476.

Shirley, J.L., de Jong, Y.P., Terhorst, C., and Herzog, R.W. (2020). Immune responses to viral gene therapy vectors. Mol Ther 28, 709-722.

Simeonov, D.R., Gowen, B.G., Boontanrart, M., Roth, T.L., Gagnon, J.D., Mumbach, M.R., Satpathy, A.T., Lee, Y., Bray, N.L., Chan, A.Y., et al. (2017). Discovery of stimulation-responsive immune enhancers with CRISPR activation. Nature 549, 111-115.

Slaymaker, I.M., Gao, L., Zetsche, B., Scott, D.A., Yan, W.X., and Zhang, F. (2016). Rationally engineered Cas 9 nucleases with improved specificity. Science $351,84-88$.

Song, C.Q., Jiang, T., Richter, M., Rhym, L.H., Koblan, L.W., Zafra, M.P., Schatoff, E.M., Doman, J.L., Cao, Y., Dow, L.E., et al. (2020). Adenine base editing in an adult mouse model of tyrosinaemia. Nat Biomed Eng $4,125-130$.

Sternberg, S.H., Redding, S., Jinek, M., Greene, E.C., and Doudna, J.A. (2014). DNA interrogation by the CRISPR RNA-guided endonuclease Cas9. Nature 507, 62-67.

Strecker, J., Jones, S., Koopal, B., Schmid-Burgk, J., Zetsche, B., Gao, L., Makarova, K.S., Koonin, E.V., and Zhang, F. (2019a). Engineering of CRISPR-Cas12b for human genome editing. Nat Commun 10, 212.

Strecker, J., Ladha, A., Gardner, Z., Schmid-Burgk, J.L., Makarova, K.S., Koonin, E.V., and Zhang, F. (2019b). RNA-guided DNA insertion with CRISPR-associated transposases. Science 365, 48-53.

Swiech, L., Heidenreich, M., Banerjee, A., Habib, N., Li, Y., Trombetta, J., Sur, M., and Zhang, F. (2015). In vivo interrogation of gene function in the mammalian brain using CRISPR-Cas9. Nat Biotechnol 33, 102106.

Tanenbaum, M.E., Gilbert, L.A., Qi, L.S., Weissman, J.S., and Vale, R.D. (2014). A protein-tagging system for signal amplification in gene expression and fluorescence imaging. Cell 159, 635-646.

Tao, D., Liu, J., Nie, X., Xu, B., Tran-Thi, T.N., Niu, L., Liu, X., Ruan, J., Lan, X., Peng, G., et al. (2020). Application of CRISPR-Cas12a enhanced fluorescence assay coupled with nucleic acid amplification for the sensitive detection of african swine fever virus. ACS Synth Biol 9, 2339-2350.

Tebas, P., Stein, D., Tang, W.W., Frank, I., Wang, S.Q., Lee, G., Spratt, S. K., Surosky, R.T., Giedlin, M.A., Nichol, G., et al. (2014). Gene editing of CCR 5 in autologous CD4 T cells of persons infected with HIV. N Engl J Med 370, 901-910.
Thompson, A.A., Walters, M.C., Kwiatkowski, J., Rasko, J.E.J., Ribeil, J. A., Hongeng, S., Magrin, E., Schiller, G.J., Payen, E., Semeraro, M., et al. (2018). Gene therapy in patients with transfusion-dependent $\beta$ thalassemia. N Engl J Med 378, 1479-1493.

Thuronyi, B.W., Koblan, L.W., Levy, J.M., Yeh, W.H., Zheng, C., Newby, G.A., Wilson, C., Bhaumik, M., Shubina-Oleinik, O., Holt, J.R., et al. (2019). Continuous evolution of base editors with expanded target compatibility and improved activity. Nat Biotechnol 37, 1070-1079.

Tian, T., Shu, B., Jiang, Y., Ye, M., Liu, L., Guo, Z., Han, Z., Wang, Z., and Zhou, X. (2021). An ultralocalized Cas13a assay enables universal and nucleic acid amplification-free single-molecule RNA diagnostics. ACS Nano 15, 1167-1178.

Tibbetts, R.S., Brumbaugh, K.M., Williams, J.M., Sarkaria, J.N., Cliby, W. A., Shieh, S.Y., Taya, Y., Prives, C., and Abraham, R.T. (1999). A role for ATR in the DNA damage-induced phosphorylation of $\mathrm{p} 53$. Genes Dev 13, 152-157.

Torikai, H., Reik, A., Liu, P.Q., Zhou, Y., Zhang, L., Maiti, S., Huls, H., Miller, J.C., Kebriaei, P., Rabinovitch, B., et al. (2012). A foundation for universal T-cell based immunotherapy: T cells engineered to express a CD19-specific chimeric-antigen-receptor and eliminate expression of endogenous TCR. Blood 119, 5697-5705.

Torikai, H., Reik, A., Soldner, F., Warren, E.H., Yuen, C., Zhou, Y., Crossland, D.L., Huls, H., Littman, N., Zhang, Z., et al. (2013). Toward eliminating HLA class I expression to generate universal cells from allogeneic donors. Blood 122, 1341-1349.

Torres-Ruiz, R., and Rodriguez-Perales, S. (2015). CRISPR-Cas9: a revolutionary tool for cancer modelling. Int J Mol Sci 16, 22151-22168.

Traxler, E.A., Yao, Y., Wang, Y.D., Woodard, K.J., Kurita, R., Nakamura, Y., Hughes, J.R., Hardison, R.C., Blobel, G.A., Li, C., et al. (2016). A genome-editing strategy to treat $\beta$-hemoglobinopathies that recapitulates a mutation associated with a benign genetic condition. Nat Med 22, 987-990.

Tsai, S.Q., Wyvekens, N., Khayter, C., Foden, J.A., Thapar, V., Reyon, D., Goodwin, M.J., Aryee, M.J., and Joung, J.K. (2014). Dimeric CRISPR RNA-guided FokI nucleases for highly specific genome editing. Nat Biotechnol 32, 569-576.

Tsai, S.Q., Zheng, Z., Nguyen, N.T., Liebers, M., Topkar, V.V., Thapar, V., Wyvekens, N., Khayter, C., Iafrate, A.J., Le, L.P., et al. (2015). GUIDEseq enables genome-wide profiling of off-target cleavage by CRISPRCas nucleases. Nat Biotechnol 33, 187-197.

Tsai, S.Q., Nguyen, N.T., Malagon-Lopez, J., Topkar, V.V., Aryee, M.J., and Joung, J.K. (2017). CIRCLE-seq: a highly sensitive in vitro screen for genome-wide CRISPR-Cas9 nuclease off-targets. Nat Methods 14, 607-614.

Tsuchiya, Y., Minami, Y., Umemura, Y., Watanabe, H., Ono, D., Nakamura, W., Takahashi, T., Honma, S., Kondoh, G., Matsuishi, T., et al. (2015). Disruption of MeCP2 attenuates circadian rhythm in CRISPR/Cas9based Rett syndrome model mouse. Genes Cells 20, 992-1005.

Urnov, F.D., Miller, J.C., Lee, Y.L., Beausejour, C.M., Rock, J.M., Augustus, S., Jamieson, A.C., Porteus, M.H., Gregory, P.D., and Holmes, M.C. (2005). Highly efficient endogenous human gene correction using designed zinc-finger nucleases. Nature 435, 646-651.

Van Duyne, G.D. (2015). Cre recombinase. Microbiol Spectr 3, MDNA3.

Veres, A., Gosis, B.S., Ding, Q., Collins, R., Ragavendran, A., Brand, H., Erdin, S., Cowan, C.A., Talkowski, M.E., and Musunuru, K. (2014). Low incidence of off-target mutations in individual CRISPR-Cas9 and TALEN targeted human stem cell clones detected by whole-genome sequencing. Cell Stem Cell 15, 27-30.

Villiger, L., Grisch-Chan, H.M., Lindsay, H., Ringnalda, F., Pogliano, C.B., Allegri, G., Fingerhut, R., Häberle, J., Matos, J., Robinson, M.D., et al. (2018). Treatment of a metabolic liver disease by in vivo genome base editing in adult mice. Nat Med 24, 1519-1525.

Villiger, L., Rothgangl, T., Witzigmann, D., Oka, R., Lin, P.J.C., Qi, W., Janjuha, S., Berk, C., Ringnalda, F., Beattie, M.B., et al. (2021). In vivo cytidine base editing of hepatocytes without detectable off-target mutations in RNA and DNA. Nat Biomed Eng 5, 179-189.

Vo, P.L.H., Ronda, C., Klompe, S.E., Chen, E.E., Acree, C., Wang, H.H., 
and Sternberg, S.H. (2021). CRISPR RNA-guided integrases for highefficiency, multiplexed bacterial genome engineering. Nat Biotechnol 39, 480-489.

Wagner, D.L., Amini, L., Wendering, D.J., Burkhardt, L.M., Akyüz, L., Reinke, P., Volk, H.D., and Schmueck-Henneresse, M. (2019). High prevalence of Streptococcus pyogenes Cas9-reactive T cells within the adult human population. Nat Med 25, 242-248.

Wallace, J., Hu, R., Mosbruger, T.L., Dahlem, T.J., Stephens, W.Z., Rao, D. S., Round, J.L., and O'Connell, R.M. (2016). Genome-wide CRISPRCas9 screen identifies microRNAs that regulate myeloid leukemia cell growth. PLoS ONE 11, e0153689.

Wang, D., Tai, P.W.L., and Gao, G. (2019a). Adeno-associated virus vector as a platform for gene therapy delivery. Nat Rev Drug Discov 18, 358378

Wang, D., Zhang, F., and Gao, G. (2020a). CRISPR-based therapeutic genome editing: strategies and in vivo delivery by AAV vectors. Cell 181, 136-150.

Wang, G., Chow, R.D., Ye, L., Guzman, C.D., Dai, X., Dong, M.B., Zhang, F., Sharp, P.A., Platt, R.J., and Chen, S. (2018a). Mapping a functional cancer genome atlas of tumor suppressors in mouse liver using AAVCRISPR-mediated direct in vivo screening. Sci Adv 4, eaao5508.

Wang, H., Yang, H., Shivalila, C.S., Dawlaty, M.M., Cheng, A.W., Zhang, F., and Jaenisch, R. (2013). One-step generation of mice carrying mutations in multiple genes by CRISPR/Cas-mediated genome engineering. Cell 153, 910-918.

Wang, J., Chen, Q., Li, S., and Li, X. (2017a). An application of a Cas protein, and a method and kit for detecting a target nucleic acid molecule. CN111094588A.

Wang, J., and Quake, S.R. (2014). RNA-guided endonuclease provides a therapeutic strategy to cure latent herpesviridae infection. Proc Natl Acad Sci USA 111, 13157-13162.

Wang, K., Jin, Q., Ruan, D., Yang, Y., Liu, Q., Wu, H., Zhou, Z., Ouyang, Z., Liu, Z., Zhao, Y., et al. (2017b). Cre-dependent Cas9-expressing pigs enable efficient in vivo genome editing. Genome Res 27, 20612071.

Wang, L., Li, L., Ma, Y., Hu, H., Li, Q., Yang, Y., Liu, W., Yin, S., Li, W., Fu, B., et al. (2020b). Reactivation of $\gamma$-globin expression through Cas9 or base editor to treat $\beta$-hemoglobinopathies. Cell Res 30, 276-278.

Wang, L., Smith, J., Breton, C., Clark, P., Zhang, J., Ying, L., Che, Y., Lape, J., Bell, P., Calcedo, R., et al. (2018b). Meganuclease targeting of PCSK9 in macaque liver leads to stable reduction in serum cholesterol. Nat Biotechnol 36, 717-725.

Wang, L., Xue, W., Yan, L., Li, X., Wei, J., Chen, M., Wu, J., Yang, B., Yang, L., and Chen, J. (2017c). Enhanced base editing by co-expression of free uracil DNA glycosylase inhibitor. Cell Res 27, 1289-1292.

Wang, L., Xue, W., Zhang, H., Gao, R., Qiu, H., Wei, J., Zhou, L., Lei, Y. N., Wu, X., Li, X., et al. (2021). Eliminating base-editor-induced genome-wide and transcriptome-wide off-target mutations. Nat Cell Biol 23, 552-563.

Wang, L., Yang, Y., Breton, C.A., White, J., Zhang, J., Che, Y., Saveliev, A., McMenamin, D., He, Z., Latshaw, C., et al. (2019b). CRISPR/Cas9mediated in vivo gene targeting corrects hemostasis in newborn and adult factor IX-knockout mice. Blood 133, 2745-2752.

Wang, M., Chen, K., Wu, Q., Peng, R., Zhang, R., and Li, J. (2020c). RCasFISH: CRISPR/dCas9-mediated in situ imaging of mRNA transcripts in fixed cells and tissues. Anal Chem 92, 2468-2475.

Wang, Q., Zhang, B., Xu, X., Long, F., and Wang, J. (2018c). CRISPRtyping PCR (ctPCR), a new Cas9-based DNA detection method. Sci Rep 8, 14126.

Wang, R., Zhao, X., Chen, X., Qiu, X., Qing, G., Zhang, H., Zhang, L., Hu, X., He, Z., Zhong, D., et al. (2020d). Rolling circular amplification (RCA)-assisted CRISPR/Cas9 cleavage (RACE) for Highly specific detection of multiple extracellular vesicle microRNAs. Anal Chem 92, 2176-2185.

Wang, T., Liu, Y., Sun, H.H., Yin, B.C., and Ye, B.C. (2019c). An RNAguided Cas9 nickase-based method for universal isothermal DNA amplification. Angew Chem Int Ed 58, 5382-5386.
Wang, T., Wei, J.J., Sabatini, D.M., and Lander, E.S. (2014). Genetic screens in human cells using the CRISPR-Cas9 system. Science 343, 80-84.

Wang, X., Huang, R., Zhang, L., Li, S., Luo, J., Gu, Y., Chen, Z., Zheng, Q., Chao, T., Zheng, W., et al. (2018d). A severe atherosclerosis mouse model on the resistant NOD background. Dis Model Mech 11.

Wang, X., Li, J., Wang, Y., Yang, B., Wei, J., Wu, J., Wang, R., Huang, X., Chen, J., and Yang, L. (2018e). Efficient base editing in methylated regions with a human APOBEC3A-Cas9 fusion. Nat Biotechnol 36, 946-949.

Wang, X., Xiong, E., Tian, T., Cheng, M., Lin, W., Wang, H., Zhang, G., Sun, J., and Zhou, X. (2020e). Clustered regularly interspaced short palindromic repeats/Cas9-mediated lateral flow nucleic acid assay. ACS Nano 14, 2497-2508.

Wang, X., Ding, C., Yu, W., Wang, Y., He, S., Yang, B., Xiong, Y.C., Wei, J., Li, J., Liang, J., et al. (2020f). Cas12a base editors induce efficient and specific editing with low DNA damage response. Cell Rep 31, 107723.

Wang, X., Yu, H., Lei, A., Zhou, J., Zeng, W., Zhu, H., Dong, Z., Niu, Y., Shi, B., Cai, B., et al. (2015a). Generation of gene-modified goats targeting MSTN and FGF5 via zygote injection of CRISPR/Cas9 system. Sci Rep 5, 13878.

Wang, Y., Du, Y., Shen, B., Zhou, X., Li, J., Liu, Y., Wang, J., Zhou, J., Hu, B., Kang, N., et al. (2015b). Efficient generation of gene-modified pigs via injection of zygote with Cas9/sgRNA. Sci Rep 5, 8256.

Wang, Y., Du, Y., Zhou, X., Wang, L., Li, J., Wang, F., Huang, Z., Huang, $\mathrm{X}$, and Wei, H. (2016). Efficient generation of B2m-null pigs via injection of zygote with TALENs. Sci Rep 6, 38854.

Weber, J., Öllinger, R., Friedrich, M., Ehmer, U., Barenboim, M., Steiger, K., Heid, I., Mueller, S., Maresch, R., Engleitner, T., et al. (2015). CRISPR/Cas9 somatic multiplex-mutagenesis for high-throughput functional cancer genomics in mice. Proc Natl Acad Sci USA 112, 13982-13987.

Weber, N.D., Stone, D., Sedlak, R.H., De Silva Feelixge, H.S., Roychoudhury, P., Schiffer, J.T., Aubert, M., and Jerome, K.R. (2014). AAV-mediated delivery of zinc finger nucleases targeting hepatitis B virus inhibits active replication. PLoS ONE 9, e97579.

Wei, J.J. (2021). Accurate and sensitive analysis of Staphylococcus aureus through CRISPR-Cas12a based recycling signal amplification cascades for early diagnosis of skin and soft tissue infections. J Microbiol Methods 183, 106167

Wei, Y., Zhou, Y., Liu, Y., Ying, W., Lv, R., Zhao, Q., Zhou, H., Zuo, E., Sun, Y., Yang, H., et al. (2021). Indiscriminate ssDNA cleavage activity of CRISPR-Cas12a induces no detectable off-target effects in mouse embryos. Protein Cell 12, 741-745.

Wernike, K., Keller, M., Conraths, F.J., Mettenleiter, T.C., Groschup, M.H., and Beer, M. (2021). Pitfalls in SARS-CoV-2 PCR diagnostics. Transbound Emerg Dis 68, 253-257.

Wilde, J.J., Aida, T., Del Rosario, R.C.H., Kaiser, T., Qi, P., Wienisch, M., Zhang, Q., Colvin, S., and Feng, G. (2021). Efficient embryonic homozygous gene conversion via RAD51-enhanced interhomolog repair. Cell 184, 3267-3280.e18.

Wilkinson, A.C., Dever, D.P., Baik, R., Camarena, J., Hsu, I., Charlesworth, C.T., Morita, C., Nakauchi, H., and Porteus, M.H. (2021). Cas9-AAV6 gene correction of beta-globin in autologous HSCs improves sickle cell disease erythropoiesis in mice. Nat Commun 12, 686.

Witzigmann, D., Kulkarni, J.A., Leung, J., Chen, S., Cullis, P.R., and van der Meel, R. (2020). Lipid nanoparticle technology for therapeutic gene regulation in the liver. Adv Drug Deliv Rev 159, 344-363.

Wong, A.S.L., Choi, G.C.G., Cui, C.H., Pregernig, G., Milani, P., Adam, M., Perli, S.D., Kazer, S.W., Gaillard, A., Hermann, M., et al. (2016). Multiplexed barcoded CRISPR-Cas9 screening enabled by CombiGEM. Proc Natl Acad Sci USA 113, 2544-2549.

Wu, W.H., Tsai, Y.T., Justus, S., Lee, T.T., Zhang, L., Lin, C.S., Bassuk, A. G., Mahajan, V.B., and Tsang, S.H. (2016). CRISPR repair reveals causative mutation in a preclinical model of retinitis pigmentosa. Mol 
Ther 24, 1388-1394.

Wu, Y., Zeng, J., Roscoe, B.P., Liu, P., Yao, Q., Lazzarotto, C.R., Clement, K., Cole, M.A., Luk, K., Baricordi, C., et al. (2019). Highly efficient therapeutic gene editing of human hematopoietic stem cells. Nat Med $25,776-783$.

Xiao, A.T., Tong, Y.X., and Zhang, S. (2020). False negative of RT-PCR and prolonged nucleic acid conversion in COVID-19: Rather than recurrence. J Med Virol 92, 1755-1756.

Xing, S., Lu, Z., Huang, Q., Li, H., Wang, Y., Lai, Y., He, Y., Deng, M., and Liu, W. (2020). An ultrasensitive hybridization chain reaction-amplified CRISPR-Cas12a aptasensor for extracellular vesicle surface protein quantification. Theranostics 10, 10262-10273.

Xiong, Y., Zhang, J., Yang, Z., Mou, Q., Ma, Y., Xiong, Y., and Lu, Y. (2020). Functional DNA regulated CRISPR-Cas12a sensors for pointof-care diagnostics of non-nucleic-acid targets. J Am Chem Soc 142, 207-213.

Xu, C., Zhou, Y., Xiao, Q., He, B., Geng, G., Wang, Z., Cao, B., Dong, X., Bai, W., Wang, Y., et al. (2021a). Programmable RNA editing with compact CRISPR-Cas13 systems from uncultivated microbes. Nat Methods 18, 499-506.

Xu, C.F., Chen, G.J., Luo, Y.L., Zhang, Y., Zhao, G., Lu, Z.D., Czarna, A., Gu, Z., and Wang, J. (2021b). Rational designs of in vivo CRISPR-Cas delivery systems. Adv Drug Deliv Rev 168, 3-29.

Xu, L., Wang, J., Liu, Y., Xie, L., Su, B., Mou, D., Wang, L., Liu, T., Wang, X., Zhang, B., et al. (2019). CRISPR-edited stem cells in a patient with HIV and acute lymphocytic leukemia. N Engl J Med 381, 1240-1247.

Xu, P., Liu, Z., Liu, Y., Ma, H., Xu, Y., Bao, Y., Zhu, S., Cao, Z., Wu, Z., Zhou, Z., et al. (2021c). Genome-wide interrogation of gene functions through base editor screens empowered by barcoded sgRNAs. Nat Biotechnol 39, 1403-1413.

Xu, P., Tong, Y., Liu, X.Z., Wang, T.T., Cheng, L., Wang, B.Y., Lv, X., Huang, Y., and Liu, D.P. (2015). Both TALENs and CRISPR/Cas9 directly target the HBB IVS2-654 (C>T) mutation in $\beta$-thalassemiaderived iPSCs. Sci Rep 5, 12065.

$\mathrm{Xu}, \mathrm{W}$. (2019). Microinjection and micromanipulation: a historical perspective. In: Liu, C., and Du, Y., eds. Microinjection. Methods in Molecular Biology. New York: Humana Press. 1-16.

Xue, W., Chen, S., Yin, H., Tammela, T., Papagiannakopoulos, T., Joshi, N. S., Cai, W., Yang, G., Bronson, R., Crowley, D.G., et al. (2014). CRISPR-mediated direct mutation of cancer genes in the mouse liver. Nature 514, 380-384.

Yan, H., Niimi, M., Matsuhisa, F., Zhou, H., Kitajima, S., Chen, Y., Wang, C., Yang, X., Yao, J., Yang, D., et al. (2020). Apolipoprotein CIII deficiency protects against atherosclerosis in knockout rabbits. Arterioscler Thromb Vasc Biol 40, 2095-2107.

Yan, S., Tu, Z., Liu, Z., Fan, N., Yang, H., Yang, S., Yang, W., Zhao, Y., Ouyang, Z., Lai, C., et al. (2018). A Huntingtin knockin pig model recapitulates features of selective neurodegeneration in Huntington's disease. Cell 173, 989-1002.e13.

Yang, B., Li, X., Lei, L., and Chen, J. (2017). APOBEC: from mutator to editor. J Genet Genomics 44, 423-437.

Yang, D., Zhang, J., Xu, J., Zhu, T., Fan, Y., Fan, J., and Chen, Y.E. (2013a). Production of apolipoprotein C-III knockout rabbits using zinc finger nucleases. J Vis Exp doi: 10.3791/50957.

Yang, H., Wang, H., Shivalila, C.S., Cheng, A.W., Shi, L., and Jaenisch, R. (2013b). One-step generation of mice carrying reporter and conditional alleles by CRISPR/Cas-mediated genome engineering. Cell 154, 13701379.

Yang, H., Gao, P., Rajashankar, K.R., and Patel, D.J. (2016). PAMdependent target DNA recognition and cleavage by C2c1 CRISPR-Cas endonuclease. Cell 167, 1814-1828.e12.

Yang, L., Yang, B., and Chen, J. (2019). One prime for all editing. Cell 179, $1448-1450$

Yang, L., Wang, L., Huo, Y., Chen, X., Yin, S., Hu, Y., Zhang, X., Zheng, R., Geng, H., Han, H., et al. (2020). Amelioration of an inherited metabolic liver disease through creation of a de novo start codon by cytidine base editing. Mol Ther 28, 1673-1683.
Ye, L., Park, J.J., Dong, M.B., Yang, Q., Chow, R.D., Peng, L., Du, Y., Guo, J., Dai, X., Wang, G., et al. (2019). In vivo CRISPR screening in CD8 T cells with AAV-Sleeping Beauty hybrid vectors identifies membrane targets for improving immunotherapy for glioblastoma. Nat Biotechnol 37, 1302-1313.

Yeh, W.H., Shubina-Oleinik, O., Levy, J.M., Pan, B., Newby, G.A., Wornow, M., Burt, R., Chen, J.C., Holt, J.R., and Liu, D.R. (2020). In vivo base editing restores sensory transduction and transiently improves auditory function in a mouse model of recessive deafness. Sci Transl Med 12.

Yin, D., Ling, S., Wang, D., Dai, Y., Jiang, H., Zhou, X., Paludan, S.R., Hong, J., and Cai, Y. (2021). Targeting herpes simplex virus with CRISPR-Cas9 cures herpetic stromal keratitis in mice. Nat Biotechnol 39, 567-577.

Yin, H., Xue, W., Chen, S., Bogorad, R.L., Benedetti, E., Grompe, M., Koteliansky, V., Sharp, P.A., Jacks, T., and Anderson, D.G. (2014). Genome editing with Cas9 in adult mice corrects a disease mutation and phenotype. Nat Biotechnol 32, 551-553.

Yin, H., Song, C.Q., Dorkin, J.R., Zhu, L.J., Li, Y., Wu, Q., Park, A., Yang, J., Suresh, S., Bizhanova, A., et al. (2016). Therapeutic genome editing by combined viral and non-viral delivery of CRISPR system components in vivo. Nat Biotechnol 34, 328-333.

Yin, H., Kauffman, K.J., and Anderson, D.G. (2017a). Delivery technologies for genome editing. Nat Rev Drug Discov 16, 387-399.

Yin, H., Song, C.Q., Suresh, S., Wu, Q., Walsh, S., Rhym, L.H., Mintzer, E., Bolukbasi, M.F., Zhu, L.J., Kauffman, K., et al. (2017b). Structureguided chemical modification of guide RNA enables potent non-viral in vivo genome editing. Nat Biotechnol 35, 1179-1187.

Yin, S., Ma, L., Shao, T., Zhang, M., Guan, Y., Wang, L., Hu, Y., Chen, X., Han, H., Shen, N., et al. (2020). Enhanced genome editing to ameliorate a genetic metabolic liver disease through co-delivery of adenoassociated virus receptor. Sci China Life Sci 65, 718-730.

Yuan, F., Guo, L., Park, K.H., Woollard, J.R., Taek-Geun, K., Jiang, K., Melkamu, T., Zang, B., Smith, S.L., Fahrenkrug, S.C., et al. (2018). Ossabaw pigs with a PCSK9 gain-of-function mutation develop accelerated coronary atherosclerotic lesions: a novel model for preclinical studies. J Am Heart Assoc 7.

Yue, H., Shu, B., Tian, T., Xiong, E., Huang, M., Zhu, D., Sun, J., Liu, Q., Wang, S., Li, Y., et al. (2021). Droplet Cas12a assay enables DNA quantification from unamplified samples at the single-molecule level. Nano Lett 21, 4643-4653.

Zabaleta, N., Barberia, M., Martin-Higueras, C., Zapata-Linares, N., Betancor, I., Rodriguez, S., Martinez-Turrillas, R., Torella, L., Vales, A., Olagüe, C., et al. (2018). CRISPR/Cas9-mediated glycolate oxidase disruption is an efficacious and safe treatment for primary hyperoxaluria type I. Nat Commun 9, 5454.

Zeng, J., Wu, Y., Ren, C., Bonanno, J., Shen, A.H., Shea, D., Gehrke, J.M., Clement, K., Luk, K., Yao, Q., et al. (2020). Therapeutic base editing of human hematopoietic stem cells. Nat Med 26, 535-541.

Zetsche, B., Gootenberg, J.S., Abudayyeh, O.O., Slaymaker, I.M., Makarova, K.S., Essletzbichler, P., Volz, S.E., Joung, J., van der Oost, J., Regev, A., et al. (2015). Cpf1 is a single RNA-guided endonuclease of a class 2 CRISPR-Cas system. Cell 163, 759-771.

Zhang, B., Wang, Q., Xu, X., Xia, Q., Long, F., Li, W., Shui, Y., Xia, X., and Wang, J. (2018a). Detection of target DNA with a novel Cas9/ sgRNAs-associated reverse PCR (CARP) technique. Anal Bioanal Chem 410, 2889-2900.

Zhang, D., Yan, Y., Que, H., Yang, T., Cheng, X., Ding, S., Zhang, X., and Cheng, W. (2020). CRISPR/Cas12a-mediated interfacial cleaving of hairpin DNA reporter for electrochemical nucleic acid sensing. ACS Sens 5, 557-562.

Zhang, J.P., Cheng, X.X., Zhao, M., Li, G.H., Xu, J., Zhang, F., Yin, M.D., Meng, F.Y., Dai, X.Y., Fu, Y.W., et al. (2019a). Curing hemophilia A by NHEJ-mediated ectopic F8 insertion in the mouse. Genome Biol 20, 276.

Zhang, K., Deng, R., Teng, X., Li, Y., Sun, Y., Ren, X., and Li, J. (2018b). Direct visualization of single-nucleotide variation in mtDNA using a 
CRISPR/Cas9-mediated proximity ligation assay. J Am Chem Soc 140, $11293-11301$.

Zhang, W., Shi, L., Zhao, Z., Du, P., Ye, X., Li, D., Cai, Z., Han, J., and Cai, J. (2019b). Disruption of CTLA-4 expression on peripheral blood CD8 ${ }^{+}$ $\mathrm{T}$ cell enhances anti-tumor efficacy in bladder cancer. Cancer Chemother Pharmacol 83, 911-920.

Zhang, W., Wan, H., Feng, G., Qu, J., Wang, J., Jing, Y., Ren, R., Liu, Z., Zhang, L., Chen, Z., et al. (2018c). SIRT6 deficiency results in developmental retardation in cynomolgus monkeys. Nature 560, 661665.

Zhang, X., Li, T., Ou, J., Huang, J., and Liang, P. (2021). Homology-based repair induced by CRISPR-Cas nucleases in mammalian embryo genome editing. Protein Cell doi: 10.1007/s13238-021-00838-7.

Zhang, X., Yue, D., Wang, Y., Zhou, Y., Liu, Y., Qiu, Y., Tian, F., Yu, Y., Zhou, Z., and Wei, W. (2019c). PASTMUS: mapping functional elements at single amino acid resolution in human cells. Genome Biol 20, 279.

Zhang, Y., Qian, L., Wei, W., Wang, Y., Wang, B., Lin, P., Liu, W., Xu, L., Li, X., Liu, D., et al. (2017a). Paired design of dCas9 as a systematic platform for the detection of featured nucleic acid sequences in pathogenic strains. ACS Synth Biol 6, 211-216.

Zhang, Y., Zhang, X., Cheng, C., Mu, W., Liu, X., Li, N., Wei, X., Liu, X., Xia, C., and Wang, H. (2017b). CRISPR-Cas9 mediated LAG-3 disruption in CAR-T cells. Front Med 11, 554-562.

Zhao, X., Zeng, L., Mei, Q., and Luo, Y. (2020a). Allosteric probe-initiated wash-free method for sensitive extracellular vesicle detection through dual cycle-assisted CRISPR-Cas12a. ACS Sens 5, 2239-2246.

Zhao, X., Zhang, G., Liu, S., Chen, X., Peng, R., Dai, L., Qu, X., Li, S., Song, H., Gao, Z., et al. (2019). Human neonatal fc receptor is the cellular uncoating receptor for enterovirus B. Cell 177, 1553-1565.e16.

Zhao, X., Zhang, W., Qiu, X., Mei, Q., Luo, Y., and Fu, W. (2020b). Rapid and sensitive exosome detection with CRISPR/Cas12a. Anal Bioanal Chem 412, 601-609.

Zheng, Q., Cai, X., Tan, M.H., Schaffert, S., Arnold, C.P., Gong, X., Chen, C.Z., and Huang, S. (2014). Precise gene deletion and replacement using the CRISPR/Cas9 system in human cells. BioTechniques 57, 115124 .

Zheng, R., Li, Y., Wang, L., Fang, X., Zhang, J., He, L., Yang, L., Li, D., and Geng, H. (2020). CRISPR/Cas9-mediated metabolic pathway reprogramming in a novel humanized rat model ameliorates primary hyperoxaluria type 1. Kidney Int 98, 947-957.

Zhong, H., Chen, Y., Li, Y., Chen, R., and Mardon, G. (2015). CRISPRengineered mosaicism rapidly reveals that loss of Kcnj13 function in mice mimics human disease phenotypes. Sci Rep 5, 8366.

Zhou, C., Sun, Y., Yan, R., Liu, Y., Zuo, E., Gu, C., Han, L., Wei, Y., Hu, X., Zeng, R., et al. (2019a). Off-target RNA mutation induced by DNA base editing and its elimination by mutagenesis. Nature 571, 275-278.

Zhou, T., Huang, M., Lin, J., Huang, R., and Xing, D. (2021). High-fidelity CRISPR/Cas13a trans-cleavage-triggered rolling circle amplified DNAzyme for visual profiling of microRNA. Anal Chem 93, 20382044.

Zhou, W., Hu, L., Ying, L., Zhao, Z., Chu, P.K., and Yu, X.F. (2018). A CRISPR-Cas9-triggered strand displacement amplification method for ultrasensitive DNA detection. Nat Commun 9, 5012.

Zhou, X., Wang, L., Du, Y., Xie, F., Li, L., Liu, Y., Liu, C., Wang, S.,
Zhang, S., Huang, X., et al. (2016). Efficient generation of genemodified pigs harboring precise orthologous human mutation via CRISPR/Cas9-induced homology-directed repair in zygotes. Hum Mutat 37, 110-118.

Zhou, X., Xin, J., Fan, N., Zou, Q., Huang, J., Ouyang, Z., Zhao, Y., Zhao, B., Liu, Z., Lai, S., et al. (2015). Generation of CRISPR/Cas9-mediated gene-targeted pigs via somatic cell nuclear transfer. Cell Mol Life Sci 72, 1175-1184.

Zhou, Y., Sharma, J., Ke, Q., Landman, R., Yuan, J., Chen, H., Hayden, D. S., Fisher Iii, J.W., Jiang, M., Menegas, W., et al. (2019b). Atypical behaviour and connectivity in SHANK3-mutant macaques. Nature 570, 326-331.

Zhou, Y., Zhu, S., Cai, C., Yuan, P., Li, C., Huang, Y., and Wei, W. (2014). High-throughput screening of a CRISPR/Cas9 library for functional genomics in human cells. Nature 509, 487-491.

Zhou, Z., and Wei, W. (2018). PrePAIRing Cas9s for screening success. Nat Biotechnol 36, 147-148.

Zhu, S., Li, W., Liu, J., Chen, C.H., Liao, Q., Xu, P., Xu, H., Xiao, T., Cao, Z., Peng, J., et al. (2016). Genome-scale deletion screening of human long non-coding RNAs using a paired-guide RNA CRISPR-Cas9 library. Nat Biotechnol 34, 1279-1286.

Zhu, S., Cao, Z., Liu, Z., He, Y., Wang, Y., Yuan, P., Li, W., Tian, F., Bao, Y., and Wei, W. (2019). Guide RNAs with embedded barcodes boost CRISPR-pooled screens. Genome Biol 20, 20.

Zhu, S., Liu, Y., Zhou, Z., Zhang, Z., Xiao, X., Liu, Z., Chen, A., Dong, X., Tian, F., Chen, S., et al. (2021). Genome-wide CRISPR activation screen identifies candidate receptors for SARS-CoV-2 entry. Sci China Life Sci 65, 701-717.

Zimmerman, P.A., Buckler-White, A., Alkhatib, G., Spalding, T., Kubofcik, J., Combadiere, C., Weissman, D., Cohen, O., Rubbert, A., Lam, G., et al. (1997). Inherited resistance to HIV-1 conferred by an inactivating mutation in $\mathrm{CC}$ chemokine receptor 5: studies in populations with contrasting clinical phenotypes, defined racial background, and quantified risk. Mol Med 3, 23-36.

Zuccaro, M.V., Xu, J., Mitchell, C., Marin, D., Zimmerman, R., Rana, B., Weinstein, E., King, R.T., Palmerola, K.L., Smith, M.E., et al. (2020). Allele-specific chromosome removal after Cas9 cleavage in human embryos. Cell 183, 1650-1664.e15.

Zuckermann, M., Hovestadt, V., Knobbe-Thomsen, C.B., Zapatka, M., Northcott, P.A., Schramm, K., Belic, J., Jones, D.T.W., Tschida, B., Moriarity, B., et al. (2015). Somatic CRISPR/Cas9-mediated tumour suppressor disruption enables versatile brain tumour modelling. Nat Commun 6, 7391.

Zuo, E., Sun, Y., Wei, W., Yuan, T., Ying, W., Sun, H., Yuan, L., Steinmetz, L.M., Li, Y., and Yang, H. (2019). Cytosine base editor generates substantial off-target single-nucleotide variants in mouse embryos. Science 364, 289-292.

Zuo, E., Sun, Y., Yuan, T., He, B., Zhou, C., Ying, W., Liu, J., Wei, W., Zeng, R., Li, Y., et al. (2020). A rationally engineered cytosine base editor retains high on-target activity while reducing both DNA and RNA off-target effects. Nat Methods 17, 600-604.

Zuris, J.A., Thompson, D.B., Shu, Y., Guilinger, J.P., Bessen, J.L., Hu, J.H., Maeder, M.L., Joung, J.K., Chen, Z.Y., and Liu, D.R. (2015). Cationic lipid-mediated delivery of proteins enables efficient protein-based genome editing in vitro and in vivo. Nat Biotechnol 33, 73-80. 